\title{
Proline-Based $N$-Oxides as Readily Available and Modular Chiral Catalysts. Enantioselective Reactions of Allyltrichlorosilane with Aldehydes
}

\author{
John F. Traverse, Yu Zhao, Amir H. Hoveyda,* and Marc L. Snapper* \\ Department of Chemistry, Merkert Chemistry Center, Boston College \\ Chestnut Hill, Massachusetts 02467
}

\section{Supporting Information}

\section{Table of Contents}

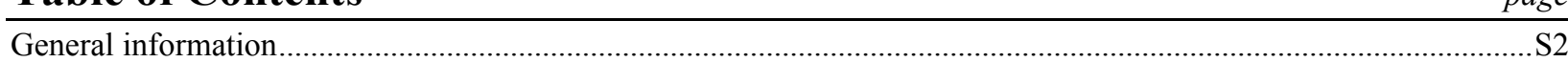

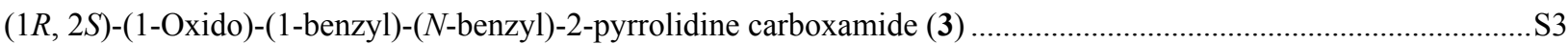

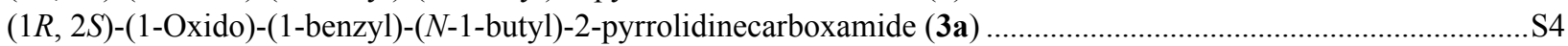

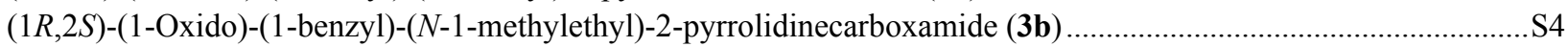

$(1 R, 2 S)$-(1-Oxido)-(1-benzyl)-( $N$-(1,1-dimethylethyl))-2-pyrrolidinecarboxamide (3c) ................................................S4

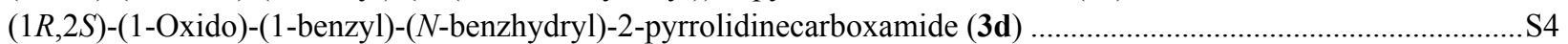

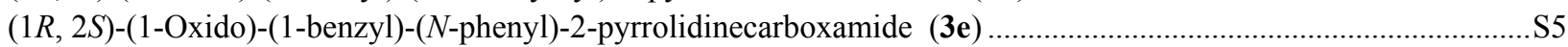

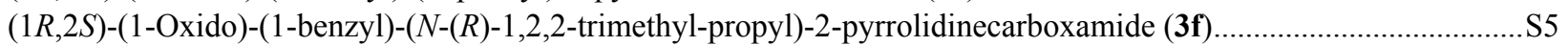

$(1 R, 2 S)$-(1-Oxido)-(1-benzyl)-( $N$-(S)-1,2,2-trimethyl-propyl)-2-pyrrolidinecarboxamide (3g) ......................................S5

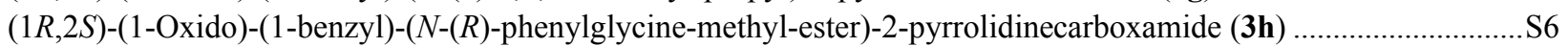

$(1 R, 2 S)$-(1-Oxido)-(1-benzyl)-( $N$-(S)-phenylglycine-methyl-ester)-2-pyrrolidinecarboxamide (3i) .............................S6

$(1 R, 2 S)$-(1-Oxido)-(1-benzyl)-( $N$-R-phenylalanine-methyl-ester)-2-pyrrolidinecarboxamide $(\mathbf{3} \mathbf{j})$................................ 6

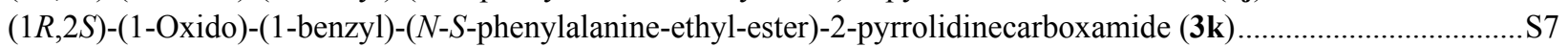

$(1 R, 2 S)-(1-O x i d o)-(1-b e n z y l)-(N-(R)-1$-phenyl-ethyl)-2-pyrrolidinecarboxamide (3I) ............................................... 7

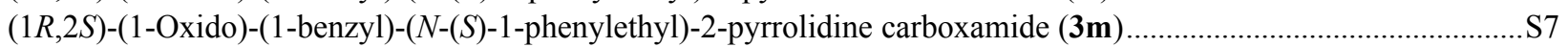

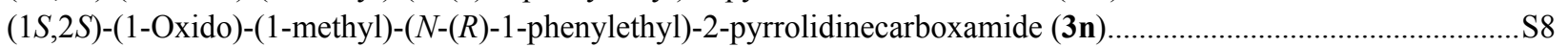

$(1 R, 2 S)-(1-O x i d o)-(1-m e t h y l e t h y l)-(N-(R)-1$-phenylethyl)-2-pyrrolidinecarboxamide (3o) ……..............................S8

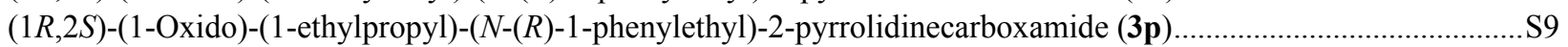

$(1 R, 2 S)$-(1-Oxido)-(1-cyclopentyl)-( $N$-(R)-1-phenylethyl)-2-pyrrolidinecarboxamide $(\mathbf{3 q})$..........................................

$(1 R, 2 S)$-(1-Oxido)-(1-cyclohexyl)-( $N$-(R)-1-phenylethyl)-2-pyrrolidinecarboxamide (3r) ……….............................S10

$(1 R, 2 S)$-(1-Oxido)-(1-tetrahydro-pyran-4-yl)-( $N$-(R)-1-phenylethyl)-2-pyrrolidinecarboxamide (3s) ........................S11

$(1 R, 2 S)$-(1-Oxido)-(1-cycloheptyl)-( $N-(R)-1$-phenylethyl)-2-pyrrolidinecarboxamide (3t) ….....................................S11

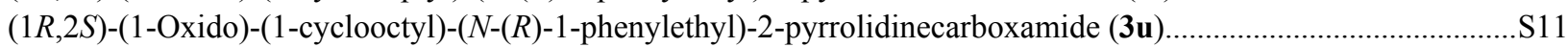

$(1 R, 2 S)$-(1-Oxido)-(1-cyclohexyl)-( $N$-( $(R)-1,2,2$-trimethyl-propyl))-2-pyrrolidinecarboxamide (3v) ..........................S12

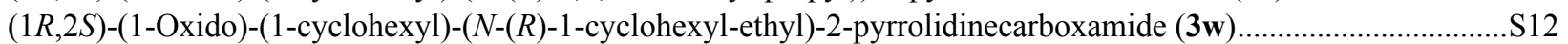

$(1 R, 2 S)$-(1-Oxido)-(1-cyclohexyl)-( $(N-(R)-1-(1,2,3,4$-tetrahydronaphthyl))-2-pyrrolidinecarboxamide (3x) ...............S12

$(1 R, 2 S)$-(1-Oxido)-(1-cyclohexyl)-( $(N-(R)-1$-(4-methoxy-phenyl-ethyl))-2-pyrrolidinecarboxamide (3y) ...................S13

$(1 R, 2 S)$-(1-Oxido)-(1-cyclohexyl)-( $(N-(R)-1$-(4-chloro-phenyl-ethyl))-2-pyrrolidinecarboxamide (3z).......................S13

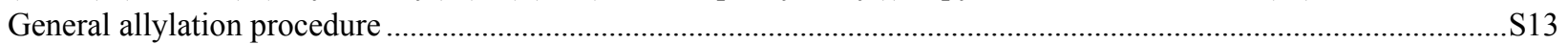

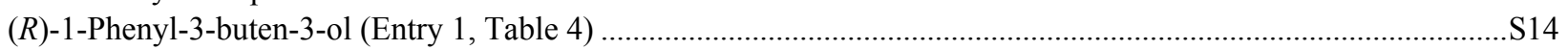

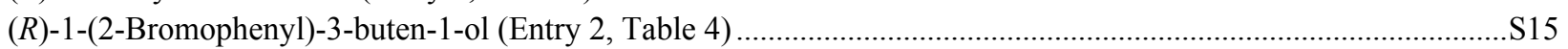

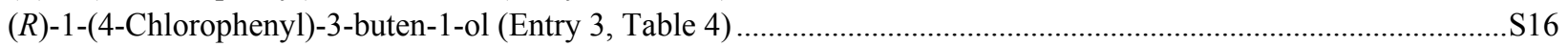

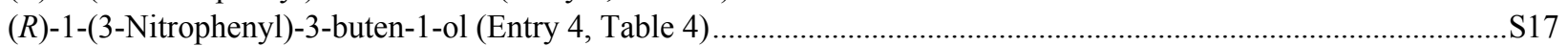

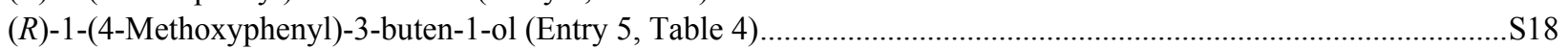

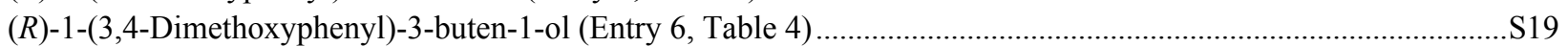

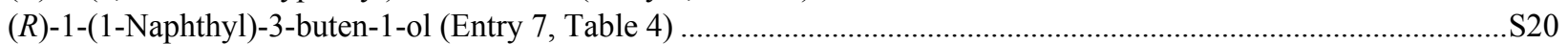

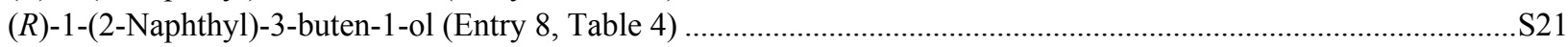

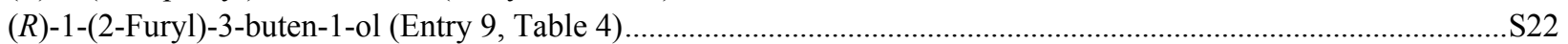




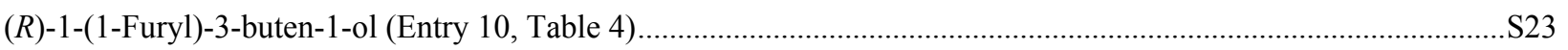

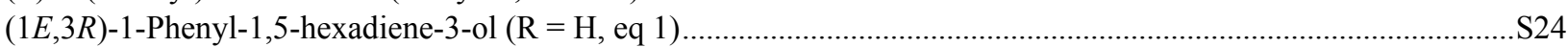

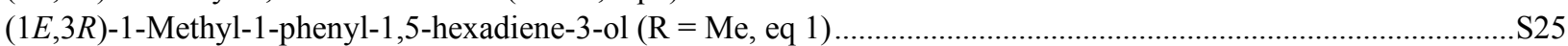

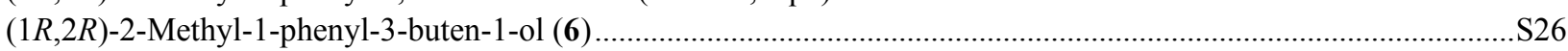

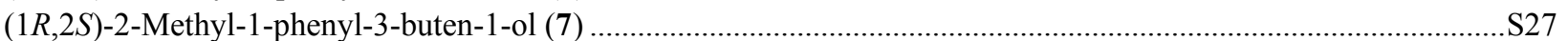

General Information. Infrared (IR) spectra were recorded on a Perkin Elmer 781 spectrophotometer, $v_{\max }$ in $\mathrm{cm}^{-1}$. The absorptions are characterized as broad (br), strong (s), medium (m), and weak (w). ${ }^{1} \mathrm{H}$ NMR were recorded on a Varian GN-400 (400 MHz). Chemical shifts are reported in ppm with the solvent reference as the internal standard $\left(\mathrm{CHCl}_{3}: \delta 7.26\right)$. Data are reported as follows: chemical shift, integration, multiplicity $(\mathrm{s}=$ singlet, $\mathrm{d}=$ doublet, $\mathrm{t}=$ triplet, $\mathrm{q}=$ quartet, $\mathrm{p}=$ pentet, hex $=$ hextet, hep $=$ heptet, $\mathrm{o}=$ octet, $\mathrm{m}=$ multiplet, $\mathrm{br}=$ broad), and coupling constants (Hz). ${ }^{13} \mathrm{C}$ NMR spectra were recorded on a Varian GN-400 (100 MHz) with complete proton decoupling. Chemical shifts are reported in ppm with the solvent reference as the internal standard $\left(\mathrm{CHCl}_{3}: \delta 77.2 \mathrm{ppm}\right)$. Melting points were taken with a Laboratory Devices Melt-Temp and are uncorrected. Enantiomeric ratios were determined by chiral HPLC or chiral GLC. Analytical liquid chromatography was performed on a Shimadzu chromatograph with a Chiracel OD (4.6 mm x 250 $\mathrm{mm}$ ) or a Chiracel AS $(4.6 \mathrm{~mm} \times 250 \mathrm{~mm})$ chiral column by Chiral Technologies. Analytical gas liquid chromatography (GLC) was performed on a Hewlett Packard 6890 with an Alpha Dex 120 (30 m x $0.25 \mathrm{~mm} \times 0.25 \mu \mathrm{m}$ film thickness), a Beta Dex 120 (30 m x $0.25 \mathrm{~mm}$ x $0.25 \mu \mathrm{m}$ film thickness) or a Gamma Dex 120 (30 m x $0.25 \mathrm{~mm}$ x $0.25 \mu \mathrm{m}$ film thickness) column by Supelco. Optical rotations were measured on a Rudolph Research Analytical Autopol IV Automatic Polarimeter. Elemental analysis (Anal) were performed by Robertson Microlit Laboratories, Inc., Madison, NJ, and are reported in percent abundance.

All reactions were conducted in oven $\left(135^{\circ} \mathrm{C}\right)$ or flame-dried glassware under an inert atmosphere of dry nitrogen. 1,2-Dichloroethane was purchased from Acros and distilled from $\mathrm{CaH}_{2}$ before use. Diethyl ether and hexanes were purchased from Fisher and distilled from $\mathrm{CaH}_{2}$ under nitrogen. Methanol, isopropanol, tetrahydrofuran and methylene chloride were purchased from Fisher and used without purification. 3-Chloroperbenzoic acid ( $m$-CPBA) (75\%) was purchased from Aldrich and used without purification. Dimethylformamide (DMF) was purchased from Fisher and was purged with nitrogen for $2 \mathrm{~h}$ and stored over $3 \AA$ molecular sieves. All amino acids and protected amino acids, $O$-benzotriazole- $N, N, N^{\prime}, N^{\prime}$-tetramethyluronium-hexafluorophosphate (HBTU), $O$-benzotriazole- $N, N, N^{\prime}, N^{\prime}$-tetramethyl uronium-tetrafluoroborate (TBTU), and 1hydroxybenzotriazole hydrate $(\mathrm{HOBt})$ were purchased from Advanced ChemTech and used without purification. Palladium on carbon (10\% dry) was purchased from Acros and used as received. All other amines for amide couplings (for ligand synthesis) were purchased from Lancaster and used without purification. All ketones (for ligand synthesis) were purchased from Aldrich and used without purification. All aldehydes were purchased from Aldrich and were either distilled (liquids) or sublimed (solids) before use. Allyltrichlorosilane was purchased from Lancaster and distilled under nitrogen before use. $(Z)$ and $(E)$ crotyltrichlorosilanes were synthesized according to literature procedure. $^{1}$

\footnotetext{
${ }^{1)}$ Iseki, K.; Kuroki, Y.; Takahashi, M.; Kishimoto, S.; Kobayashi, Y. Tetrahedron, 1997, 53, 3513-3526.
} 


\section{Representative procedure for the synthesis of Proline Based N-Oxide Ligands.}

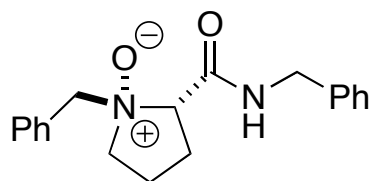

(1R,2S)-(1-Oxido)-(1-benzyl)-( $N$-benzyl)-2-pyrrolidine carboxamide (3). Step 1: ${ }^{2}$ To a dry $250 \mathrm{~mL}$ flask, $L$-proline $(17.3 \mathrm{~g}, 0.150 \mathrm{~mol})$ and potassium hydroxide $(25.4 \mathrm{~g}, 0.450 \mathrm{~mol})$ were dissolved in isopropanol $(100 \mathrm{~mL})$ and heated to $40^{\circ} \mathrm{C}$. Benzyl chloride $(18.9 \mathrm{~mL}, 0.16 \mathrm{~mol})$ was added over three hours via syringe pump. The reaction was stirred for an additional $6 \mathrm{~h}$ (nine hours total), at which time the reaction was allowed to cool to room temperature. Concentrated $\mathrm{HCl}$ was added to reach a $\mathrm{pH}$ of $4-5$. Chloroform $(40 \mathrm{~mL})$ was added and the reaction was allowed to stir at room temperature for $12 \mathrm{~h}$. The reaction was filtered to remove the white precipitate. The precipitate was washed with chloroform $(3 \times 20 \mathrm{~mL})$. The solvent was removed from the combined organic layers to yield a yellow solid, which was washed with acetone (4 x $50 \mathrm{~mL})$. The solid was dried over $\mathrm{P}_{2} \mathrm{O}_{5}$ to yield the unpurified benzylated acid (29.2 g, 95\% unpurified yield).

Step 2: A portion of the unpurified benzylated acid $(1.0 \mathrm{~g}, 5.0 \mathrm{mmol})$ was dissolved in DMF $(25 \mathrm{~mL})$ and THF $(10 \mathrm{~mL})$. To this solution was added benzylamine $(586 \mu \mathrm{L}, 5.00 \mathrm{mmol})$, potassium carbonate $(2.10 \mathrm{~g}, 15.0$ mmol), and HBTU (1.9 g, $5.0 \mathrm{mmol})$. The reaction was allowed to stir for $3 \mathrm{~h}$, after which time water $(50 \mathrm{~mL})$ was added. The crude mixture was extracted with ethyl acetate $(2 \times 50 \mathrm{~mL})$. The organic layer was washed with $1 \%$ aqueous sodium carbonate $(5 \times \mathrm{x} 50 \mathrm{~mL})$ and brine $\left(\begin{array}{llll}1 & \mathrm{x} & 25 & \mathrm{~mL}\end{array}\right)$, dried over anhydrous $\mathrm{Na}_{2} \mathrm{SO}_{4}$, filtered, and concentrated under reduced pressure to yield the unpurified amide (1.1 g, 76\% unpurified yield).

Step 3: ${ }^{3}$ The unpurified benzyl amide $(1.1 \mathrm{~g}, 3.7 \mathrm{mmol})$ was dissolved in methylene chloride $(50 \mathrm{~mL})$. Potassium carbonate $(1.0 \mathrm{~g}, 7.5$ mmol) was added and the reaction cooled to $-78{ }^{\circ} \mathrm{C}$. $m$-CPBA $(0.86 \mathrm{~g}$ of $75 \%$ pure, net $0.65 \mathrm{~g}, 3.7 \mathrm{mmol}$ ) was then added, and the reaction was allowed to stir at $-78{ }^{\circ} \mathrm{C}$ for $3 \mathrm{~h}$. At this time, the reaction was allowed to warm to room temperature. After stirring for $2 \mathrm{~h}$ at room temperature, methylene chloride $(50 \mathrm{~mL})$ was added to dilute the reaction and celite $(500 \mathrm{mg})$ was added to aid filtration. The reaction was filtered, and methylene chloride was removed under reduced pressure. The resulting oil was diluted with methylene chloride/hexanes from which pure $\mathbf{3}$ was crystallized as an off-white solid (735 mg, 45\% yield over 3 steps). M.P. 119-120 ${ }^{\circ} \mathrm{C}$. IR (neat, thin film): 2957 (br), 1673 (s), 1542 (s), 1499 (w), 1350 (w), 748 (m), 698 (s) cm ${ }^{-1} .{ }^{1} \mathrm{H} \mathrm{NMR}\left(\mathrm{CDCl}_{3}, 400 \mathrm{MHz}\right)$ : $\delta 11.64$ (1H, br), 7.42-7.39 (5H, m), 7.36-7.25 (4H, m), $7.24(1 \mathrm{H}, \mathrm{tt}, J=6.0,2.8 \mathrm{~Hz})$, 4.49-4.45 (4 H, m), $3.66(1 \mathrm{H}, \mathrm{dd}, J=11.2,7.6 \mathrm{~Hz}), 3.32(1 \mathrm{H}, \mathrm{p}, J=10.8 \mathrm{~Hz}), 3.25(1 \mathrm{H}, \mathrm{tt}, J=$ 8.4, $2.4 \mathrm{~Hz}), 2.52-2.32(3 \mathrm{H}, \mathrm{m}), 1.88-1.80(1 \mathrm{H}, \mathrm{m}) .{ }^{13} \mathrm{C} \mathrm{NMR}\left(\mathrm{CDCl}_{3}, 100 \mathrm{MHz}\right): \delta 167.8,138.6$, 132.2, 130.0, 129.8, 128.9, 127.6, 127.2, 72.4, 71.0, 66.7, 42.9, 27.6, 20.1. Anal Calcd for $\mathrm{C}_{19} \mathrm{H}_{22} \mathrm{~N}_{2} \mathrm{O}_{2}$ : C, 73.52; H, 7.14; N, 9.03. Found C, 73.28; H, 7.41; N, 9.32. Optical Rotation: $[\alpha]^{25}$ $-43^{\circ}$ (c $\left.1.0, \mathrm{CHCl}_{3}\right)$.

\footnotetext{
${ }^{2}$ Adapted from Belokon', Y. N.; Tararov, V. I.; Maleev, V. I.; Savel'eva, T. F.; Ryzhov, M. G. Tetrahedron: Asymmetry, 1998, 9, 4249-4252.

3) O’Neil, I. A.; Potter, A. J. Chem. Commun. 1998, 1487-1488 and references cited therein.
} 


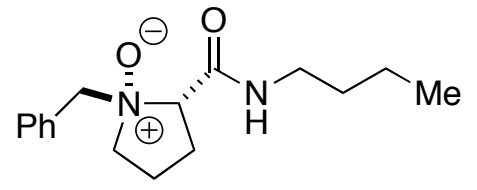

(1R,2S)-(1-Oxido)-(1-benzyl)-( $N$-1-butyl)-2-pyrrolidinecarboxamide (3a). The same general procedure (modified for scale) as $\mathbf{3}$ was followed, only substituting $n$-butylamine $(618 \mu \mathrm{L}, 6.00 \mathrm{mmol})$ for benzylamine in step 2. Unpurified 3a was purified by silica gel chromatography $\left(97: 3 \mathrm{CH}_{2} \mathrm{Cl}_{2}: \mathrm{MeOH}\right)$ to yield pure $\mathbf{3 a}(729 \mathrm{mg}, 51 \%$ yield, 3 steps) as a pale yellow solid. M.P. $64-65^{\circ}$ C. IR (neat, thin film): $2958(\mathrm{~m}), 2927(\mathrm{~m})$, 2864 (w), 1660 (s), 1560 (s), 1454 (m), 748 (w), 705 (m) cm ${ }^{-1} .{ }^{1} \mathrm{H} \mathrm{NMR}\left(\mathrm{CDCl}_{3}, 400 \mathrm{MHz}\right)$ : ठ $11.04(1 \mathrm{H}, \mathrm{br}), 7.43(5 \mathrm{H}, \mathrm{br}), 4.51(1 \mathrm{H}, \mathrm{d}, J=12.8 \mathrm{~Hz}), 4.46(1 \mathrm{H}, \mathrm{d}, J=12.8 \mathrm{~Hz}), 3.63(1 \mathrm{H}, \mathrm{t}, J=$ $8.0 \mathrm{~Hz}), 3.37-3.24(4 \mathrm{H}, \mathrm{m}), 2.48-2.30(3 \mathrm{H}, \mathrm{m}), 1.87-1.79(1 \mathrm{H}, \mathrm{m}), 1.58-1.51(2 \mathrm{H}, \mathrm{m}), 1.40(2 \mathrm{H}, \mathrm{h}, J$ $=7.2 \mathrm{~Hz}), 0.94(3 \mathrm{H}, \mathrm{t}, J=7.2 \mathrm{~Hz}) .{ }^{13} \mathrm{C} \mathrm{NMR}\left(\mathrm{CDCl}_{3}, 100 \mathrm{MHz}\right): \delta 167.7,132.3,130.1,129.9$, 129.0, 72.4, 71.0, 66.7, 38.7, 31.9, 27.6, 20.7, 20.1, 14.1. Anal Calcd for $\mathrm{C}_{16} \mathrm{H}_{24} \mathrm{~N}_{2} \mathrm{O}_{2}$ : $\mathrm{C}, 69.53 ; \mathrm{H}$, 8.75; N, 10.14. Found C, 69.30; H, 8.77; N, 10.03. Optical Rotation: $[\alpha]^{25}{ }_{\mathrm{D}}-15^{\circ}\left(\mathrm{c} 1.0, \mathrm{CHCl}_{3}\right)$.

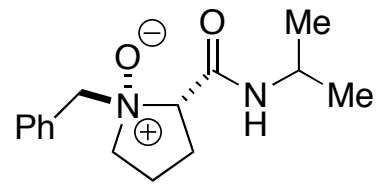

$(1 R, 2 S)-(1-O x i d o)-(1-b e n z y)-(N$-1-methylethyl)-2-pyrrolidinecarboxamide (3b). The same general procedure as $\mathbf{3}$ was followed, only substituting isopropylamine $(415 \mu \mathrm{L}, 5.00 \mathrm{mmol})$ for benzylamine in step 2. Unpurified product $\mathbf{3 b}$ was purified by silica gel chromatography (97:3 $\left.\mathrm{CH}_{2} \mathrm{Cl}_{2}: \mathrm{MeOH}\right)$ to yield pure $\mathbf{3 b}(465 \mathrm{mg}, 34 \%$ yield, 3 steps) as an off-white solid. M.P. 129-130 ${ }^{\circ} \mathrm{C}$. IR (neat, thin film): 2955 (br), 1665 (s), 1551 (s), 1457 (w), 1369 (w), 841 (w), 752 (m), 715 (m), $664(\mathrm{w}) \mathrm{cm}^{-1} .{ }^{1} \mathrm{H}$ NMR $\left(\mathrm{CDCl}_{3}, 400 \mathrm{MHz}\right): \delta 10.99(1 \mathrm{H}, \mathrm{d}, J=7.2 \mathrm{~Hz}), 7.42(5 \mathrm{H}, \mathrm{br}), 4.60$ $(1 \mathrm{H}, \mathrm{d}, J=13.2 \mathrm{~Hz}), 4.45(1 \mathrm{H}, \mathrm{d}, J=13.2 \mathrm{~Hz}), 4.08(1 \mathrm{H}, \mathrm{o}, J=6.8 \mathrm{~Hz}), 3.58(1 \mathrm{H}, \mathrm{dd}, J=10.8,7.6$ $\mathrm{Hz}), 3.32(1 \mathrm{H}, \mathrm{q}, J=9.6 \mathrm{~Hz}), 3.24(1 \mathrm{H}, \mathrm{dt}, J=8.4,1.6 \mathrm{~Hz}), 2.46-2.29(3 \mathrm{H}, \mathrm{m}), 1.86-1.79(1 \mathrm{H}, \mathrm{m})$,

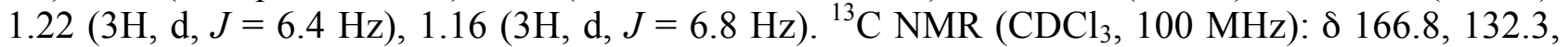
130.0, 129.9, 129.0, 72.2, 70.9, 66.6, 41.0, 27.5, 23.2, 22.8, 20.1. Anal Calcd for $\mathrm{C}_{15} \mathrm{H}_{22} \mathrm{~N}_{2} \mathrm{O}_{2}: \mathrm{C}$, 68.67; H, 8.45; N, 10.68. Found C, 68.40; H, 8.80; N, 10.48. Optical Rotation: $[\alpha]^{25}{ }_{\mathrm{D}}-14^{\circ}(\mathrm{c} 1.0$, $\left.\mathrm{CHCl}_{3}\right)$.

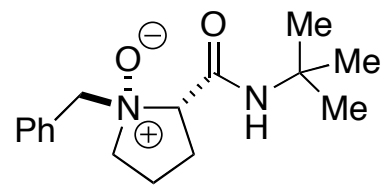

(1R,2S)-(1-Oxido)-(1-benzyl)-( $N$-(1,1-dimethylethyl))-2-pyrrolidinecarboxamide (3c). The same general procedure (modified for scale) as $\mathbf{3}$ was followed, only substituting $t$-butylamine $(661 \mu \mathrm{L}, 6.00 \mathrm{mmol})$ for benzylamine in step 2. Unpurified 3c was purified by silica gel chromatography $\left(97: 3 \mathrm{CH}_{2} \mathrm{Cl}_{2}: \mathrm{MeOH}\right)$ to yield pure $3 \mathrm{c}(632 \mathrm{mg}, 45 \%$ yield, $3 \mathrm{steps})$ as a white solid. M.P. $92-94{ }^{\circ}$ C. IR (neat, thin film): 2958 (br), 1666 (s), 1566 (s), 1454 (w), 1378 (w), 1229 (m), $755(\mathrm{w}), 711(\mathrm{~m}) \mathrm{cm}^{-1} .{ }^{1} \mathrm{H}$ NMR $\left(\mathrm{CDCl}_{3}, 400 \mathrm{MHz}\right): \delta 11.01(1 \mathrm{H}, \mathrm{s}), 7.42(5 \mathrm{H}, \mathrm{br}), 4.47(2 \mathrm{H}$, s), $3.45(1 \mathrm{H}, \mathrm{t}, J=8.0 \mathrm{~Hz}), 3.30(1 \mathrm{H}, \mathrm{dd}, J=19.6,10.4 \mathrm{~Hz}), 3.22(1 \mathrm{H}, \mathrm{dt}, J=8.4,1.6 \mathrm{~Hz}), 2.45-$ $2.28(3 \mathrm{H}, \mathrm{m}), 1.84-1.76(1 \mathrm{H}, \mathrm{m}), 1.39(9 \mathrm{H}, \mathrm{s}) .{ }^{13} \mathrm{C} \mathrm{NMR}\left(\mathrm{CDCl}_{3}, 100 \mathrm{MHz}\right): \delta$ 166.5, 132.3, 130.1, 129.8, 129.0, 72.6, 70.8, 66.4, 50.8, 29.1, 27.4, 20.0. Anal Calcd for $\mathrm{C}_{16} \mathrm{H}_{24} \mathrm{~N}_{2} \mathrm{O}_{2}$ : C, 69.53; H, 8.75; $\mathrm{N}, 10.14$. Found C, 69.27; H, 9.00; N, 10.25. Optical Rotation: $[\alpha]^{25}-8.8^{\circ}\left(\mathrm{c} 0.5, \mathrm{CHCl}_{3}\right)$.

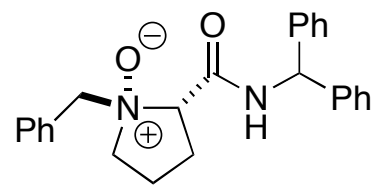

(1R,2S)-(1-Oxido)-(1-benzyl)-( $N$-benzhydryl)-2-pyrrolidinecarboxamide (3d). The same general procedure (modified for scale) as $\mathbf{3}$ was followed, only substituting aminodiphenylmethane $(840 \mu \mathrm{L}, 5.0 \mathrm{mmol})$ for benzylamine in step 2 . Unpurified 3d was purified by silica gel chromatography (97:3 $\left.\mathrm{CH}_{2} \mathrm{Cl}_{2}: \mathrm{MeOH}\right)$ to yield pure 3d (1.12 g, 57\% yield, 3 steps) as a white solid. M.P. 122-124 ${ }^{\circ} \mathrm{C}$. IR (neat, thin film): 3062 (w), 3031 (w), 2949 (w), 1671 (s), 1533 (m), 1495 (m), 1451 (w), $752(\mathrm{~m}), 702(\mathrm{~s}), 551(\mathrm{w}) \mathrm{cm}^{-1} .{ }^{1} \mathrm{H} \mathrm{NMR}\left(\mathrm{CDCl}_{3}, 400 \mathrm{MHz}\right): \delta 12.62(1 \mathrm{H}, \mathrm{d}, J=8.8 \mathrm{~Hz}), 7.43-$ 
$7.21(15 \mathrm{H}, \mathrm{m}), 6.32(1 \mathrm{H}, \mathrm{d}, J=8.8 \mathrm{~Hz}), 4.46(1 \mathrm{H}, \mathrm{d}, J=13.6 \mathrm{~Hz}), 4.33(1 \mathrm{H}, \mathrm{d}, J=13.6 \mathrm{~Hz}), 3.66$ $(1 \mathrm{H}, \mathrm{dd}, J=11.6,8.4 \mathrm{~Hz}), 3.34(1 \mathrm{H}, \mathrm{q}, J=10.0 \mathrm{~Hz}), 3.23(1 \mathrm{H}, \mathrm{dt}, J=8.8,2.4 \mathrm{~Hz}), 2.56-2.32(3 \mathrm{H}$, $\mathrm{m}), 1.90-1.81(1 \mathrm{H}, \mathrm{m}) .{ }^{13} \mathrm{C} \mathrm{NMR}\left(\mathrm{CDCl}_{3}, 100 \mathrm{MHz}\right): \delta 166.9,142.5,142.0,132.2,130.0,129.8$, $128.9,128.8,128.6,127.4,127.3,127.1,127.0,72.6,70.7,66.4,56.7,27.4,20.2$. Anal Calcd for $\mathrm{C}_{25} \mathrm{H}_{26} \mathrm{~N}_{2} \mathrm{O}_{2}$ : C, 77.69; H, 6.78; N, 7.25. Found C, 77.47; H, 6.99; N, 7.06. Optical Rotation: $[\alpha]^{25}$ $-63^{\circ}\left(\mathrm{c} 1.0, \mathrm{CHCl}_{3}\right)$.

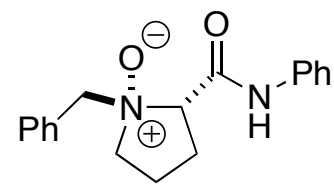

(1R,2S)-(1-Oxido)-(1-benzyl)-( $N$-phenyl)-2-pyrrolidinecarboxamide (3e). The same general procedure (modified for scale) as $\mathbf{3}$ was followed, only substituting aniline $(570 \mu \mathrm{L}, 5.00 \mathrm{mmol})$ for benzylamine in step 2 . Unpurified $3 \mathbf{e}$ was purified by silica gel chromatography (97:3 $\left.\mathrm{CH}_{2} \mathrm{Cl}_{2}: \mathrm{MeOH}\right)$ to yield pure $\mathbf{3 e}(738 \mathrm{mg}, 48 \%$ yield, 3 steps $)$ as an off white solid. M.P. 152-153 ${ }^{\circ} \mathrm{C}$. IR (neat, thin film): 2955 (br), 1671 (s), 1596 (m), 1558 (s), 1501 (w), 1445 (m), 1325 (w), 759 (m), $702(\mathrm{~m}), 532(\mathrm{w}) \mathrm{cm}^{-1} .{ }^{1} \mathrm{H}$ NMR $\left(\mathrm{CDCl}_{3}, 400 \mathrm{MHz}\right): \delta 13.75(1 \mathrm{H}, \mathrm{s}), 7.63(2 \mathrm{H}, \mathrm{dd}, J=7.6,1.2$ $\mathrm{Hz}), 7.48-7.45(2 \mathrm{H}, \mathrm{m}), 7.41-7.38(3 \mathrm{H}, \mathrm{m}), 7.32(2 \mathrm{H}, \mathrm{t}, J=7.6 \mathrm{~Hz}), 7.09(1 \mathrm{H}, \mathrm{tt}, J=7.6,1.2 \mathrm{~Hz})$, $4.56(1 \mathrm{H}, \mathrm{d}, J=13.2 \mathrm{~Hz}), 4.52(1 \mathrm{H}, \mathrm{d}, J=13.2 \mathrm{~Hz}), 3.70(1 \mathrm{H}, \mathrm{dd}, J=11.2,7.6 \mathrm{~Hz}), 3.41-3.36(2 \mathrm{H}$, $\mathrm{m}), 2.60-2.37(3 \mathrm{H}, \mathrm{m}), 1.96-1.84(1 \mathrm{H}, \mathrm{m}) .{ }^{13} \mathrm{C} \mathrm{NMR}\left(\mathrm{CDCl}_{3}, 100 \mathrm{MHz}\right): \delta 165.6,138.1,132.3$, 130.0, 129.8, 129.0, 128.9, 124.1, 120.3, 72.6, 71.3, 66.9, 27.6, 20.3. Anal Calcd for $\mathrm{C}_{18} \mathrm{H}_{20} \mathrm{~N}_{2} \mathrm{O}_{2}$ : C, 72.95; H, 6.80; N, 9.45. Found C, 72.79; H, 7.04; N, 9.44. Optical Rotation: $[\alpha]^{25}-75^{\circ}$ (c 1.0 , $\left.\mathrm{CHCl}_{3}\right)$.

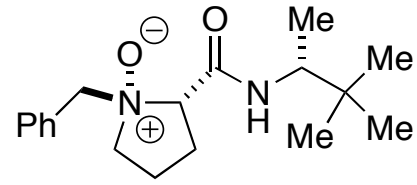

(1R,2S)-(1-Oxido)-(1-benzyl)-( $N$-(R)-1,2,2-trimethyl-propyl)-2-pyrrolidinecarboxamide (3f). The same general procedure (modified for scale) as 3 was followed, only substituting $(R)$-3,3-dimethyl-2butylamine $(652 \mu \mathrm{L}, 5.00 \mathrm{mmol})$ for benzylamine in step 2 . Unpurified 3f was purified by silica gel chromatography $\left(97: 3 \mathrm{CH}_{2} \mathrm{Cl}_{2}: \mathrm{MeOH}\right)$ to yield pure $\mathbf{3 f}(875 \mathrm{mg}, 56 \%$ yield, 3 steps) as a sticky white solid. M.P. 178-180 ${ }^{\circ}$ C. IR (neat, thin film): $2970(\mathrm{~s}), 2870(\mathrm{~m})$, 1666 (s), 1549 (s), 1455 (m), 1369 (m), 1133 (w), $754(\mathrm{~m}), 698(\mathrm{~m}), 661(\mathrm{~m}), 493(\mathrm{w}) \mathrm{cm}^{-1} .{ }^{1} \mathrm{H}$ NMR $\left(\mathrm{CDCl}_{3}, 400 \mathrm{MHz}\right): \delta 11.40(1 \mathrm{H}, \mathrm{d}, J=7.2 \mathrm{~Hz}), 7.45-7.41(5 \mathrm{H}, \mathrm{m}), 4.52(1 \mathrm{H}, \mathrm{d}, J=12.8 \mathrm{~Hz})$, $4.41(1 \mathrm{H}, \mathrm{d}, J=12.8 \mathrm{~Hz}), 3.86(1 \mathrm{H}, \mathrm{dq}, J=9.2,6.8 \mathrm{~Hz}), 3.59(1 \mathrm{H}, \mathrm{t}, J=8.4 \mathrm{~Hz}), 3.33(1 \mathrm{H}, \mathrm{dd}, J=$ 20.4, $9.6 \mathrm{~Hz}), 3.23(1 \mathrm{H}, J=8.4,2.0 \mathrm{~Hz}), 2.51-2.30(3 \mathrm{H}, \mathrm{m}), 1.87-1.78(1 \mathrm{H}, \mathrm{m}), 1.14(3 \mathrm{H}, \mathrm{d}, J=6.8$ $\mathrm{Hz}), 0.95(9 \mathrm{H}, \mathrm{s}) .{ }^{13} \mathrm{C} \mathrm{NMR}\left(\mathrm{CDCl}_{3}, 100 \mathrm{MHz}\right): \delta 166.8,132.3,130.2,129.9,72.7,70.9,66.5,53.0$, 34.6, 27.7, 26.7, 20.1, 16.6. Anal Calcd for $\mathrm{C}_{18} \mathrm{H}_{28} \mathrm{~N}_{2} \mathrm{O}_{2}$ : C, 71.02; H, 9.27; N, 9.20. Found $\mathrm{C}$, 70.77; H, 9.34; N, 8.90. Optical Rotation: $[\alpha]_{\mathrm{D}}^{25}-25^{\circ}\left(\mathrm{c} 1.0, \mathrm{CHCl}_{3}\right)$.

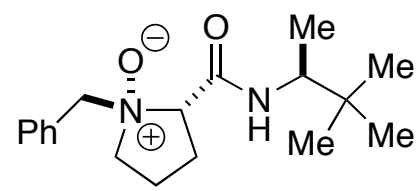

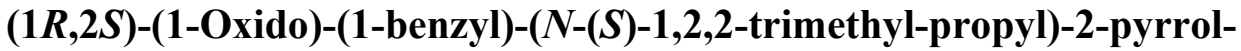
idinecarboxamide $\mathbf{( 3 g )}$. The same general procedure (modified for scale) as 3 was followed, only substituting $(S)$-3,3-dimethyl-2-butylamine (652 $\mu \mathrm{L}, 5.00 \mathrm{mmol}$ ) for benzylamine in step 2. Unpurified $\mathbf{3 g}$ was purified by silica gel chromatography $\left(97: 3 \mathrm{CH}_{2} \mathrm{Cl}_{2}: \mathrm{MeOH}\right)$ to yield pure $\mathbf{3 g}(890 \mathrm{mg}$, 59\% yield, 3 steps) as a white solid. M.P. 137-138 ${ }^{\circ} \mathrm{C}$. IR (neat, thin film): 2964 (s), 2864 (w), 1666 (s), 1549 (m), $1511(\mathrm{~m}), 1449$ (w), 1362 (w), $1213(\mathrm{w}), 1133$ (w), $748(\mathrm{~m}), 704(\mathrm{~m}) \mathrm{cm}^{-1} .{ }^{1} \mathrm{H}$ NMR $\left(\mathrm{CDCl}_{3}\right.$, $400 \mathrm{MHz}): \delta 11.36(1 \mathrm{H}, \mathrm{d}, J=8.0 \mathrm{~Hz}), 7.45-7.42(5 \mathrm{H}, \mathrm{m}), 4.59(1 \mathrm{H}, \mathrm{d}, J=12.8 \mathrm{~Hz}), 4.47(1 \mathrm{H}, \mathrm{d}, J$ $=12.8 \mathrm{~Hz}), 3.91(1 \mathrm{H}, \mathrm{ddd}, J=13.6,9.2,6.8 \mathrm{~Hz}), 3.61(1 \mathrm{H}, \mathrm{dd}, J=11.2,7.6 \mathrm{~Hz}), 3.30(1 \mathrm{H}, \mathrm{q}, J=$ $10.0 \mathrm{~Hz}), 3.17(1 \mathrm{H}, \mathrm{dt}, J=8.4,1.6 \mathrm{~Hz}), 2.48-2.30(3 \mathrm{H}, \mathrm{m}), 1.86-1.78(1 \mathrm{H}, \mathrm{m}), 1.13(3 \mathrm{H}, \mathrm{d}, J=6.4$ $\mathrm{Hz}), 1.02(9 \mathrm{H}, \mathrm{s}) .{ }^{13} \mathrm{C} \mathrm{NMR}\left(\mathrm{CDCl}_{3}, 100 \mathrm{MHz}\right): \delta 167.0,132.2,130.2,129.8,129.0,72.5,70.8$, 
66.1, 53.3, 34.3, 27.6, 27.0, 20.1, 16.3. Anal Calcd for $\mathrm{C}_{18} \mathrm{H}_{28} \mathrm{~N}_{2} \mathrm{O}_{2}$ : C, 71.02; H, 9.27; N, 9.20. Found C, 70.80; H, 9.46; N, 9.10. Optical Rotation: $[\alpha]^{25}{ }_{D}+15^{\circ}\left(\mathrm{c} 1.0, \mathrm{CHCl}_{3}\right)$.

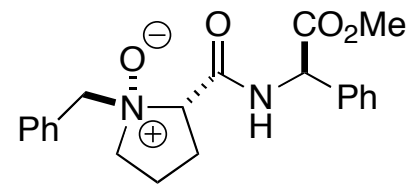

(1R,2S)-(1-Oxido)-(1-benzyl)-( $N$-(R)-phenylglycine-methyl-ester)-2pyrrolidinecarboxamide $(\mathbf{3 h})$. The same general procedure as $\mathbf{3}$ was followed, only substituting $(R)$-phenylglycine methyl ester $(0.98 \mathrm{~g}, 5.0$ mmol) for benzylamine in step 2 . Unpurified product $\mathbf{3 h}$ was purified by silica gel chromatography $\left(98: 2 \mathrm{CH}_{2} \mathrm{Cl}_{2}: \mathrm{MeOH}\right)$ to yield pure $\mathbf{3 h}(784 \mathrm{mg}, 42 \%$ yield, 3 steps $)$ as a white solid. M.P. $134-135^{\circ} \mathrm{C}$. IR (neat, thin film): 2961 (w), 1747 (s), 1678 (s), 1532 (m), 1457 (w), 1338 (w), 1269 (w), 1206 (m), 1174 (w), 1017 (w), $759(\mathrm{~m}), 721(\mathrm{~m}), 589$ (w) cm ${ }^{-1} .{ }^{1} \mathrm{H}$ NMR $\left(\mathrm{CDCl}_{3}, 400 \mathrm{MHz}\right): \delta 12.56(1 \mathrm{H}, \mathrm{d}, J=6.8 \mathrm{~Hz}), 7.48(2 \mathrm{H}, \mathrm{d}, J=7.2 \mathrm{~Hz}), 7.39-7.28(8 \mathrm{H}, \mathrm{m}), 5.51$ $(1 \mathrm{H}, \mathrm{d}, J=7.2 \mathrm{~Hz}), 4.43(1 \mathrm{H}, \mathrm{d}, J=12.8 \mathrm{~Hz}), 3.71(3 \mathrm{H}, \mathrm{s}), 3.62(1 \mathrm{H}, \mathrm{dd}, J=10.8,7.6 \mathrm{~Hz}), 3.39-$ $3.26(2 \mathrm{H}, \mathrm{m}), 2.68-2.58(1 \mathrm{H}, \mathrm{m}), 2.51-2.34(2 \mathrm{H}, \mathrm{m}), 1.92-1.82(1 \mathrm{H}, \mathrm{m}) .{ }^{13} \mathrm{C} \mathrm{NMR}\left(\mathrm{CDCl}_{3}, 100\right.$ $\mathrm{MHz}): \delta 170.7,167.7,136.4,132.2,129.9,129.8,129.0,128.9,128.4,127.5,72.3,70.9,67.0,56.8$, 52.8, 27.5, 20.2. Anal Calcd for $\mathrm{C}_{21} \mathrm{H}_{24} \mathrm{~N}_{2} \mathrm{O}_{4}$ : C, 68.46; $\mathrm{H}, 6.57 ; \mathrm{N}, 7.60$. Found $\mathrm{C}, 68.27 ; \mathrm{H}, 6.90$; $\mathrm{N}, 7.47$. Optical Rotation: $[\alpha]^{25}-128^{\circ}\left(\mathrm{c} 1.0, \mathrm{CHCl}_{3}\right)$.

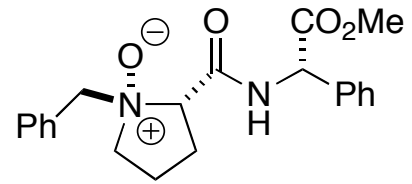

$(1 R, 2 S)-(1-O x i d o)-(1-b e n z y l)-(N-(S)$-phenylglycine-methyl-ester)-2pyrrolidinecarboxamide (3i). The same general procedure (modified for scale) as 3 was followed, only substituting $(S)$-phenylglycine methyl ester $(0.98 \mathrm{~g}, 5.0 \mathrm{mmol})$ for benzylamine in step 2. Unpurified product $3 \mathbf{i}$ was purified by silica gel chromatography $\left(98: 2 \mathrm{CH}_{2} \mathrm{Cl}_{2}: \mathrm{MeOH}\right)$ to yield pure $3 \mathbf{i}$ (492 $\mathrm{mg}$, 26\% yield, 3 steps) as a white solid. M.P. 159-161 ${ }^{\circ} \mathrm{C}$. IR (neat, thin film): 2949 (m), 1747 (s), 1671 (s), 1533 (m), $1464(\mathrm{w}), 1331(\mathrm{w}), 1212(\mathrm{~m}), 1168(\mathrm{w}), 752(\mathrm{~m}), 702(\mathrm{~m}), 589(\mathrm{w}) \mathrm{cm}^{-1} .{ }^{1} \mathrm{H}$ NMR $\left(\mathrm{CDCl}_{3}\right.$, $400 \mathrm{MHz}): \delta 12.77(1 \mathrm{H}, \mathrm{d}, J=7.2 \mathrm{~Hz}), 7.52-7.30(10 \mathrm{H}, \mathrm{m}), 5.56(1 \mathrm{H}, \mathrm{d}, J=7.2 \mathrm{~Hz}), 4.95(1 \mathrm{H}, \mathrm{d}, J$ $=12.8 \mathrm{~Hz}), 4.73(1 \mathrm{H}, \mathrm{d}, J=12.8 \mathrm{~Hz}), 3.74(3 \mathrm{H}, \mathrm{s}), 3.63(1 \mathrm{H}, \mathrm{dd}, 11.2,8.0 \mathrm{~Hz}) 3.37(1 \mathrm{H}, \mathrm{q}, J=9.6$ $\mathrm{Hz}), 3.26(1 \mathrm{H}, \mathrm{dt}, J=8.0,1.6 \mathrm{~Hz}), 2.46-2.25(3 \mathrm{H}, \mathrm{m}), 1.86-1.80(1 \mathrm{H}, \mathrm{m}) .{ }^{13} \mathrm{C} \mathrm{NMR}\left(\mathrm{CDCl}_{3}, 100\right.$ $\mathrm{MHz}): \delta 171.5,168.1,135.6,132.5,130.4,129.8,129.2,129.0,128.6,127.6,71.9,70.5,65.8,57.1$, 52.9, 27.0, 20.3. Anal Calcd for $\mathrm{C}_{21} \mathrm{H}_{24} \mathrm{~N}_{2} \mathrm{O}_{4}$ : C, 68.46; H, 6.57; N, 7.60. Found C, 68.08; H, 6.92; N, 7.43. Optical Rotation: $[\alpha]^{25}+64^{\circ}\left(\right.$ c $\left.1.0, \mathrm{CHCl}_{3}\right)$.

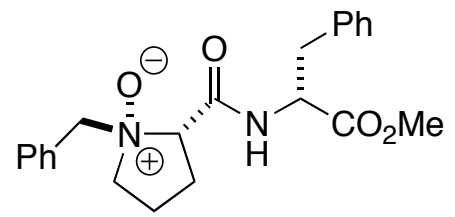

(1R,2S)-(1-Oxido)-(1-benzyl)-( $N$-R-phenylalanine-methyl-ester)-2pyrrolidinecarboxamide $(\mathbf{3 j})$. The same general procedure (modified for scale) as $\mathbf{3}$ was followed, only substituting $(R)$-phenylalanine methyl ester (733 mg, $3.40 \mathrm{mmol})$ for benzylamine in step 2 . Unpurified 3j was purified by silica gel chromatography (99.5:0.5 $\left.\mathrm{CH}_{2} \mathrm{Cl}_{2}: \mathrm{MeOH}\right)$ to yield pure $\mathbf{3 j}(992 \mathrm{mg}, 52 \%$ yield, 3 steps $)$ as a white solid. M.P. $136-137{ }^{\circ} \mathrm{C}$. IR (neat, thin film): 2951 (br), 1740 (s), 1666 (s), 1536 (s), 1493 (w), 1455 (m), 1369 (w), 1220 (m), $754(\mathrm{~m}), 704(\mathrm{~s}) \mathrm{cm}^{-1} .{ }^{1} \mathrm{H} \mathrm{NMR}\left(\mathrm{CDCl}_{3}, 400 \mathrm{MHz}\right): \delta 11.89(1 \mathrm{H}, \mathrm{d}, J=7.6 \mathrm{~Hz}), 7.41-7.33(5 \mathrm{H}, \mathrm{m})$, $7.27(2 \mathrm{H}, \mathrm{t}, J=8.0 \mathrm{~Hz}), 7.15-7.11(3 \mathrm{H}, \mathrm{m}), 4.89(1 \mathrm{H}, \mathrm{ddd}, J=10.4,7.6,4.4 \mathrm{~Hz}), 4.17(1 \mathrm{H}, \mathrm{d}, J=$ $13.2 \mathrm{~Hz}), 3.91(1 \mathrm{H}, \mathrm{d}, J=13.2 \mathrm{~Hz}), 3.76(3 \mathrm{H}, \mathrm{s}), 3.45-3.34(2 \mathrm{H}, \mathrm{m}), 3.25-3.13(2 \mathrm{H}, \mathrm{m}), 3.02(1 \mathrm{H}$, $\mathrm{dd}, J=13.6,10.4 \mathrm{~Hz}), 2.52-2.21(3 \mathrm{H}, \mathrm{m}), 1.82-1.74(1 \mathrm{H}, \mathrm{m}) .{ }^{13} \mathrm{C} \mathrm{NMR}\left(\mathrm{CDCl}_{3}, 100 \mathrm{MHz}\right): \delta$ $171.7,168.0,137.1,132.2,130.1,128.7,129.3,128.8,128.7,127.0,72.0,70.3,66.2,53.4,52.6$, 37.9, 27.2, 20.1. Anal Calcd for $\mathrm{C}_{22} \mathrm{H}_{26} \mathrm{~N}_{2} \mathrm{O}_{4}$ : C, 69.09; H, 6.85; N, 7.32. Found C, 68.90; H, 6.86; $\mathrm{N}, 7.24$. Optical Rotation: $[\alpha]_{\mathrm{D}}^{25}-1.0^{\circ}\left(\mathrm{c} 1.0, \mathrm{CHCl}_{3}\right)$. 


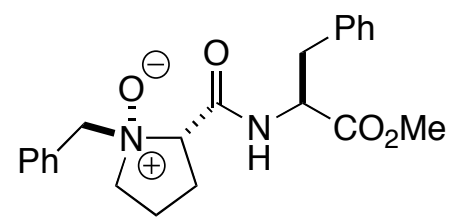

$(1 R, 2 S)-(1-O x i d o)-(1-b e n z y l)-(N-S$-phenylalanine-ethyl-ester)-2pyrrolidinecarboxamide (3k). The same general procedure (modified for scale) as 3 was followed, only substituting $(S)$-phenylalanine ethyl ester (783 mg, $3.40 \mathrm{mmol}$ ) for benzylamine in step 2. Unpurified $\mathbf{3 k}$ was purified by silica gel chromatography $\left(97: 3 \mathrm{CH}_{2} \mathrm{Cl}_{2}: \mathrm{MeOH}\right)$ to yield pure 3k (728 mg, 51\% yield, 3 steps) as a yellow oil which solidified upon standing. M.P. 46$47{ }^{\circ} \mathrm{C}$. IR (neat, thin film): 2964 (br), 1741 (s), 1666 (s), 1536 (m), 1499 (w), 1220 (m), 1189 (m), $1027(\mathrm{w}), 748(\mathrm{~m}), 698(\mathrm{~s}) \mathrm{cm}^{-1} .{ }^{1} \mathrm{H} \mathrm{NMR}\left(\mathrm{CDCl}_{3}, 400 \mathrm{MHz}\right): \delta 12.15(1 \mathrm{H}, \mathrm{d}, J=8.0 \mathrm{~Hz}), 7.40-$ $7.35(5 \mathrm{H}, \mathrm{m}), 7.30-7.24(4 \mathrm{H}, \mathrm{m}), 7.20(1 \mathrm{H}, \mathrm{tt}, J=7.2,2.0 \mathrm{~Hz}), 4.81(1 \mathrm{H}, \mathrm{dq}, J=8.8,4.8 \mathrm{~Hz}), 4.62$ $(1 \mathrm{H}, \mathrm{d}, J=13.2 \mathrm{~Hz}), 4.57(1 \mathrm{H}, \mathrm{d}, J=13.2 \mathrm{~Hz}), 4.19(1 \mathrm{H}, \mathrm{d}, J=7.2 \mathrm{~Hz}), 4.16(1 \mathrm{H}, \mathrm{d}, J=7.2 \mathrm{~Hz})$, $3.49(1 \mathrm{H}, \mathrm{dd}, J=11.2,8.0 \mathrm{~Hz}), 3.31-3.16(3 \mathrm{H}, \mathrm{m}), 3.07(1 \mathrm{H}, \mathrm{dd}, J=14.0,9.2 \mathrm{~Hz}), 2.35-2.11(3 \mathrm{H}$, $\mathrm{m}), 1.80-1.77(1 \mathrm{H}, \mathrm{m}), 1.21(3 \mathrm{H}, \mathrm{t}, J=7.2 \mathrm{~Hz}) .{ }^{13} \mathrm{C} \mathrm{NMR}\left(\mathrm{CDCl}_{3}, 100 \mathrm{MHz}\right): \delta 171.3,167.9$, $136.5,132.1,130.2,129.4,129.2,128.6,128.3,126.7,72.1,69.9,65.6,61.3,53.4,37.8,26.8,19.9$, 14.3. Anal Calcd for $\mathrm{C}_{23} \mathrm{H}_{28} \mathrm{~N}_{2} \mathrm{O}_{4}$ : C, 69.67; H, 7.12; N, 7.07. Found C, 69.46; H, 7.39; N, 6.98. Optical Rotation: $[\alpha]^{25}-27^{\circ}\left(\mathrm{c} 1.0, \mathrm{CHCl}_{3}\right)$.

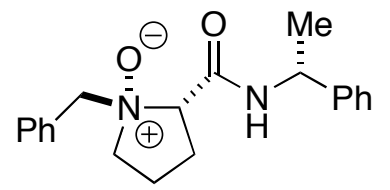

(1R,2S)-(1-Oxido)-(1-benzyl)-( $N$-(R)-1-phenyl-ethyl)-2-pyrrolidinecarboxamide (3I). The same general procedure as $\mathbf{3}$ was followed, only substituting $(R)-1$-phenylethylamine $(620 \mu \mathrm{L}, 5.0 \mathrm{mmol})$ for benzylamine in step 2. Unpurified 3I was purified by silica gel chromatography (97:3 $\left.\mathrm{CH}_{2} \mathrm{Cl}_{2}: \mathrm{MeOH}\right)$ to yield pure 31 ( $895 \mathrm{mg}, 54 \%$ yield, 3 steps) as a white solid. M.P. $138-139{ }^{\circ} \mathrm{C}$. IR (neat, thin film): 2964 (br), 1666 (s), 1547 (s), 1497 (w), 1441 (w), 755 (m), 698 (s) cm ${ }^{-1} .{ }^{1} \mathrm{H}$ NMR $\left(\mathrm{CDCl}_{3}, 400 \mathrm{MHz}\right): \delta 11.83(1 \mathrm{H}, \mathrm{d}, J=8.0 \mathrm{~Hz}), 7.49-6.42(5 \mathrm{H}, \mathrm{m}), 7.36-7.30(4 \mathrm{H}, \mathrm{m}), 7.23(1 \mathrm{H}, \mathrm{tt}$, $J=6.8,2.0 \mathrm{~Hz}), 5.12(1 \mathrm{H}, \mathrm{p}, J=6.8 \mathrm{~Hz}), 4.51(1 \mathrm{H}, \mathrm{d}, J=13.2 \mathrm{~Hz}), 4.50(1 \mathrm{H}, \mathrm{d}, J=13.2 \mathrm{~Hz}), 3.62$ $(1 \mathrm{H}, \mathrm{dd}, J=10.8,8.0 \mathrm{~Hz}), 3.35(1 \mathrm{H}, \mathrm{q}, J=9.6 \mathrm{~Hz}), 3.26(1 \mathrm{H}, \mathrm{dt}, J=10.8,8.4 \mathrm{~Hz}), 2.45-2.26(3 \mathrm{H}$, $\mathrm{m}), 1.86-1.79(1 \mathrm{H}, \mathrm{m}), 1.51(3 \mathrm{H}, \mathrm{d}, J=6.8 \mathrm{~Hz}) .{ }^{13} \mathrm{C} \mathrm{NMR}\left(\mathrm{CDCl}_{3}, 100 \mathrm{MHz}\right): \delta 166.8,143.8$, 132.4, 130.2, 129.9, 129.0, 128.7, 127.0, 126.1, 72.5, 71.1, 66.9, 48.7, 27.6, 23.7, 20.1. Anal Calcd for $\mathrm{C}_{20} \mathrm{H}_{24} \mathrm{~N}_{2} \mathrm{O}_{2}: \mathrm{C}, 74.04 ; \mathrm{H}, 7.46 ; \mathrm{N}, 8.64$. Found C, 73.81; H, 7.69; N, 8.64. Optical Rotation: $[\alpha]_{\mathrm{D}}^{25}+9.4^{\circ}\left(\mathrm{c} 1.0, \mathrm{CHCl}_{3}\right)$.

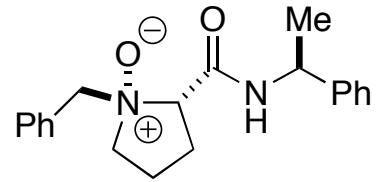

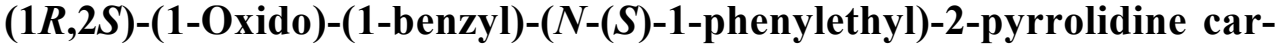
boxamide (3m). The same general procedure (modified for scale) as $\mathbf{3}$ was followed, only substituting $(S)$-1-phenylethylamine $(620 \mu \mathrm{L}, 5.0 \mathrm{mmol})$ for benzylamine in step 2. Unpurified $\mathbf{3} \mathbf{m}$ was purified by silica gel chromatography (97:3 $\left.\mathrm{CH}_{2} \mathrm{Cl}_{2}: \mathrm{MeOH}\right)$ to yield pure $\mathbf{3 m}(596 \mathrm{mg}, 36 \%$ yield, 3 steps) as an offwhite solid. M.P. $123-124{ }^{\circ} \mathrm{C}$. IR (neat, thin film): 2967 (br), 1672 (s), 1540 (s), 1497 (w), 1459 (w), $770(\mathrm{~m}), 708(\mathrm{~m}), 501(\mathrm{~m}) \mathrm{cm}^{-1} .{ }^{1} \mathrm{H} \mathrm{NMR}\left(\mathrm{CDCl}_{3}, 400 \mathrm{MHz}\right): \delta 11.88(1 \mathrm{H}, \mathrm{d}, J=8.0 \mathrm{~Hz}), 7.45$ $(2 \mathrm{H}, \mathrm{dd}, J=8.4,1.6 \mathrm{~Hz}), 7.40-7.31(5 \mathrm{H}, \mathrm{m}), 7.27-7.21(3 \mathrm{H}, \mathrm{m}), 5.17(1 \mathrm{H}, \mathrm{p}, J=6.8 \mathrm{~Hz}), 4.41(1 \mathrm{H}$, $\mathrm{d}, J=12.8 \mathrm{~Hz}), 4.29(1 \mathrm{H}, \mathrm{d}, J=12.8 \mathrm{~Hz}), 5.54(1 \mathrm{H}, \mathrm{dd}, J=11.6,8.0 \mathrm{~Hz}), 3.30(1 \mathrm{H}, \mathrm{q}, J=10.0 \mathrm{~Hz})$, $3.21(1 \mathrm{H}, \mathrm{dt}, J=8.4,2.4 \mathrm{~Hz}), 2.55-2.31(3 \mathrm{H}, \mathrm{m}), 1.92-1.81(1 \mathrm{H}, \mathrm{m}), 1.52(3 \mathrm{H}, \mathrm{d}, J=7.2 \mathrm{~Hz}) .{ }^{13} \mathrm{C}$ NMR $\left(\mathrm{CDCl}_{3}, 100 \mathrm{MHz}\right): \delta 166.8,144.1,132.2,130.0,129.8,129.0,128.7,127.1,126.3,72.3$, 70.8, 66.4, 48.8, 27.4, 23.2, 20.2. Anal Calcd for $\mathrm{C}_{20} \mathrm{H}_{24} \mathrm{~N}_{2} \mathrm{O}_{2}$ : C, 74.04; $\mathrm{H}, 7.46 ; \mathrm{N}$, 8.64. Found $\mathrm{C}$, 74.07; H, 7.60; N, 8.51. Optical Rotation: $[\alpha]^{25}-84^{\circ}$ (c 1.0, $\left.\mathrm{CHCl}_{3}\right)$. 


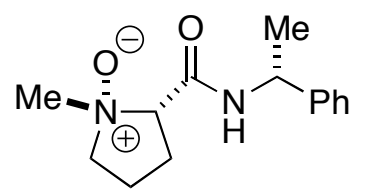

(1S,2S)-(1-Oxido)-(1-methyl)-( $N$-(R)-1-phenylethyl)-2-pyrrolidinecarboxamide (3n). ( $S)$ - $N$-Methylproline ${ }^{4}(0.65 \mathrm{~g}, 5.0 \mathrm{mmol}),(R)-1$ phenylethylamine $(0.64 \mathrm{~mL}, 5.0 \mathrm{mmol})$, and $\mathrm{K}_{2} \mathrm{CO}_{3}(1.0 \mathrm{~g}, 7.5 \mathrm{mmol})$ were added to DMF $(20 \mathrm{~mL})$ and THF $(15 \mathrm{~mL})$ and were stirred for 30 minutes to dissolve the acid. HBTU $(2.1 \mathrm{~g}, 5.5 \mathrm{mmol})$ was added and the reaction was allowed to stir for $2 \mathrm{~h}$ after which time water $(50 \mathrm{~mL})$ was added. The reaction was extracted with ethyl acetate $(2 \times 50$ $\mathrm{mL})$. The organic layer was washed with $1 \%$ aqueous sodium carbonate $(5 \mathrm{x} 50 \mathrm{~mL})$ and brine $(1 \mathrm{x}$ $25 \mathrm{~mL}$ ), dried over anhydrous $\mathrm{Na}_{2} \mathrm{SO}_{4}$, filtered, and concentrated under reduced pressure to yield unpurified amide (0.96 g, 83\% unpurified yield).

The unpurified amide $(0.96 \mathrm{~g}, 4.1 \mathrm{mmol})$ was dissolved in methylene chloride $(60 \mathrm{~mL})$. Potassium carbonate $(1.1 \mathrm{~g}, 8.3 \mathrm{mmol})$ was then added and the reaction was cooled to $-78^{\circ} \mathrm{C}$. $m$-CPBA $(0.95 \mathrm{~g}$ of $75 \%$ pure, net $0.71 \mathrm{~g}, 4.1 \mathrm{mmol})$ was then added, and the reaction was allowed to stir at $-78^{\circ} \mathrm{C}$ for $3 \mathrm{~h}$. At this time, the reaction was allowed to warm to room temperature. After stirring for $2 \mathrm{~h}$ at room temperature, methylene chloride $(50 \mathrm{~mL})$ was added to dilute the reaction and celite $(500 \mathrm{mg})$ was added to aid filtration. The reaction was then filtered, and the methylene chloride was removed under reduced pressure. The resulting oil was purified by silica gel chromatography $\left(94: 6 \mathrm{CH}_{2} \mathrm{Cl}_{2}: \mathrm{MeOH}\right)$ to yield pure $\mathbf{3 n}$ as a off white solid $(688 \mathrm{mg}, 56 \%$ yield over 2 steps from $N$-methylproline). M.P. $101-103{ }^{\circ} \mathrm{C}$. IR (neat, thin film): 2962 (br), 1671 (s), 1545 (s), $1495(\mathrm{w}), 1445(\mathrm{~m}), 1212(\mathrm{w}), 866(\mathrm{w}), 765(\mathrm{~m}), 696(\mathrm{~m}), 513(\mathrm{w}) \mathrm{cm}^{-1} .{ }^{1} \mathrm{H} \mathrm{NMR}\left(\mathrm{CDCl}_{3}\right.$, $400 \mathrm{MHz}): \delta 11.71(1 \mathrm{H}, \mathrm{d}, J=7.6 \mathrm{~Hz}), 7.38-7.19(5 \mathrm{H}, \mathrm{m}), 5.12(1 \mathrm{H}, \mathrm{p}, J=6.8 \mathrm{~Hz}), 3.68(1 \mathrm{H}, \mathrm{t}, J=$ $8.4 \mathrm{~Hz}), 3.59(1 \mathrm{H}, \mathrm{tt}, J=8.4,2.0 \mathrm{~Hz}), 3.46(1 \mathrm{H}, \mathrm{q}, J=9.6 \mathrm{~Hz}), 3.33(3 \mathrm{H}, \mathrm{s}), 2.50-2.35(3 \mathrm{H}, \mathrm{m})$, 2.06-1.95 (1H, m), $1.51(3 \mathrm{H}, \mathrm{d}, J=7.2 \mathrm{~Hz}) .{ }^{13} \mathrm{C} \mathrm{NMR}\left(\mathrm{CDCl}_{3}, 100 \mathrm{MHz}\right): \delta 166.3,143.6,128.7$, 127.1, 126.0, 76.1, 71.6, 55.5, 48.7, 28.2, 23.5, 20.7. Anal Calcd for $\mathrm{C}_{14} \mathrm{H}_{20} \mathrm{~N}_{2} \mathrm{O}_{2}$ : C, 67.71; H, 8.12; $\mathrm{N}, 11.28$. Found C, 67.56; H, 8.09; N, 11.12. Optical Rotation: $[\alpha]_{\mathrm{D}}^{25}+35^{\circ}$ (c 1.0, $\mathrm{CHCl}_{3}$ ).

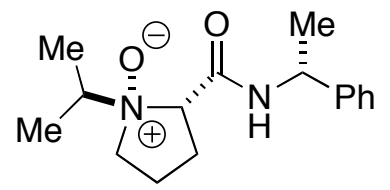

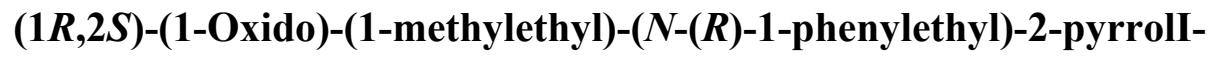 dinecarboxamide (3o).}

Step 1: Palladium on Carbon ( $10 \%$ by wt., dry, $0.12 \mathrm{~g})$ was added to a dry $100 \mathrm{~mL}$ flask and flushed with $\mathrm{N}_{2}$. Methanol $(20 \mathrm{~mL})$ was then slowly added. To this suspension was charged $(S)$-proline $(1.2 \mathrm{~g}, 10 \mathrm{mmol})$ and acetone $(3.7 \mathrm{~mL}$, $50 \mathrm{mmol}$ ). The flask was charged with $\mathrm{H}_{2}$ and was kept under a balloon of $\mathrm{H}_{2}$ for $16 \mathrm{~h}$. At this time the reaction was purged with $\mathrm{N}_{2}$ and filtered through celite. Removal of the methanol under reduced pressure led to the unpurified acid (1.5 g, 96\% unpurified yield).

Step 2: The unpurified acid $(1.5 \mathrm{~g}, 50 \mathrm{mmol})$ was dissolved in DMF $(40 \mathrm{~mL})$ and THF $(30 \mathrm{~mL})$. (R)-Phenylethylamine $(1.2 \mathrm{~mL}, 9.6 \mathrm{mmol})$ and $\mathrm{K}_{2} \mathrm{CO}_{3}(2.0 \mathrm{~g}, 14$ mmol) were added and the mixture was allowed to stir for 30 minutes to dissolve the acid. TBTU (3.4 g, $11 \mathrm{mmol})$ was then added and the reaction was allowed to stir for $2 \mathrm{~h}$ after which time water $(50 \mathrm{~mL})$ was added. The reaction was extracted with ethyl acetate $(2 \times 50 \mathrm{~mL})$. The organic layer was washed with $1 \%$ aqueous sodium carbonate $(5 \times 50 \mathrm{~mL})$ and brine $\left(\begin{array}{llll}1 & \mathrm{x} & 25 \mathrm{~mL}\end{array}\right)$, dried over anhydrous $\mathrm{Na}_{2} \mathrm{SO}_{4}$, filtered, and concentrated under reduced pressure to yield $1.99 \mathrm{~g}$ of unpurified amide (79\% unpurified yield).

Step 3: The unpurified amide $(1.5 \mathrm{~g}, 5.8 \mathrm{mmol})$ was then dissolved in methylene chloride $(80 \mathrm{~mL})$. Potassium carbonate $(1.60 \mathrm{~g}, 11.5 \mathrm{mmol})$ was then added and the reaction was cooled to $-78{ }^{\circ} \mathrm{C}$. $m$-CPBA $(1.3 \mathrm{~g}$ of $75 \%$ pure, net $0.99 \mathrm{~g}, 5.8$

\footnotetext{
${ }^{4}$ Synthesized from $(S)$-proline and formaldehyde, see Aurelio, L.; Box, J. S.; Brownlee, R. T. C.; Hughes, A. B.; Sleebs, M. M. J. Org. Chem. 2003, 68, 2652-2667.
} 
mmol) was then added, and the reaction was allowed to stir at -78 ${ }^{\circ} \mathrm{C}$ for $3 \mathrm{~h}$. At this time, the reaction was allowed to warm to room temperature. After stirring for 2 $\mathrm{h}$ at room temperature, methylene chloride $(50 \mathrm{~mL})$ was added to dilute the reaction and celite $(500 \mathrm{mg})$ was added to aid filtration. The reaction was then filtered, and the methylene chloride was removed under reduced pressure. The resulting oil was purified by silica gel chromatography $\left(96: 4 \mathrm{CH}_{2} \mathrm{Cl}_{2}: \mathrm{MeOH}\right)$ to yield pure 30 as an off white solid $\left(1.1 \mathrm{~g}, 52 \%\right.$ yield over 3 steps). M.P. $113-114{ }^{\circ} \mathrm{C}$. IR (neat, thin film): 2970 (br), 1660 (s), 1547 (s), 1491 (w), 1441 (w), 1372 (w), 761 (w), $698(\mathrm{~m}) \mathrm{cm}^{-1} .{ }^{1} \mathrm{H} \mathrm{NMR}\left(\mathrm{CDCl}_{3}, 400 \mathrm{MHz}\right): \delta 11.77(1 \mathrm{H}, \mathrm{d}, J=7.6 \mathrm{~Hz}), 7.37-7.20(5 \mathrm{H}, \mathrm{m}), 5.09$ $(1 \mathrm{H}, \mathrm{p}, J=6.8 \mathrm{~Hz}), 3.65(1 \mathrm{H}, \mathrm{t}, J=8.8 \mathrm{~Hz}), 3.53(1 \mathrm{H}, \mathrm{p}, J=6.4 \mathrm{~Hz}), 3.42-3.37(1 \mathrm{H}, \mathrm{m}), 3.23(1 \mathrm{H}$, dd, $J=19.2,8.8 \mathrm{~Hz}), 2.46-2.33(3 \mathrm{H}, \mathrm{m}), 1.95-1.87(1 \mathrm{H}, \mathrm{m}), 1.464(3 \mathrm{H}, \mathrm{d}, J=6.8 \mathrm{~Hz}), 1.457(3 \mathrm{H}$, $\mathrm{d}, J=6.8 \mathrm{~Hz}), 3.39(3 \mathrm{H}, \mathrm{d}, J=6.4 \mathrm{~Hz}) .{ }^{13} \mathrm{C} \mathrm{NMR}\left(\mathrm{CDCl}_{3}, 100 \mathrm{MHz}\right): \delta 167.9,143.0,128.6,126.9$, 126.0, 73.3, 68.5, 66.6, 48.6, 29.3, 29.3, 23.2, 20.5, 18.3. Anal Calcd for $\mathrm{C}_{16} \mathrm{H}_{24} \mathrm{~N}_{2} \mathrm{O}_{2}$ : C, 69.53; $\mathrm{H}, 8.75 ; \mathrm{N}, 10.14$. Found $\mathrm{C}, 69.58 ; \mathrm{H}, 8.81 ; \mathrm{N}, 9.98$. Optical Rotation: $[\alpha]_{\mathrm{D}}^{25}+1.8^{\circ}$ (c 1.0 , $\left.\mathrm{CHCl}_{3}\right)$.

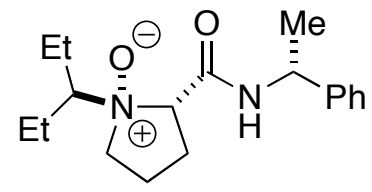

(1R,2S)-(1-Oxido)-(1-ethylpropyl)-( $N$-(R)-1-phenylethyl)-2-pyrrolIdinecarboxamide (3p). 3p was synthesized in the same manner as 3o (adjusted for scale) but with 3 -pentanone $(6.3 \mathrm{~mL}, 60 \mathrm{mmol})$ instead of acetone in step 1. Unpurified 3p was purified by silica gel chromatography (98:2 $\left.\mathrm{CH}_{2} \mathrm{Cl}_{2}: \mathrm{MeOH}\right)$ to yield pure $\mathbf{3 p}$ as a white solid (1.4 g, 53\% yield). M.P. 110-111 ${ }^{\circ} \mathrm{C}$. IR (neat, thin film): 2970 (br), 2877 (w), 1660 (s), 1542 (s), 1493 (m), 1449 (m), 1374 (w), $1213(\mathrm{w}), 1126(\mathrm{w}), 1021(\mathrm{w}), 878(\mathrm{w}), 760(\mathrm{w}), 704(\mathrm{~m}), 524(\mathrm{w}) \mathrm{cm}^{-1} .{ }^{1} \mathrm{H} \mathrm{NMR}\left(\mathrm{CDCl}_{3}, 400\right.$ $\mathrm{MHz}): \delta 11.95(1 \mathrm{H}, \mathrm{d}, J=8.0 \mathrm{~Hz}), 7.31-7.19(5 \mathrm{H}, \mathrm{m}), 5.12(1 \mathrm{H}, \mathrm{dq}, J=6.8,1.2 \mathrm{~Hz}), 3.74(1 \mathrm{H}, \mathrm{t}, J$ $=8.0 \mathrm{~Hz}), 3.39-3.25(2 \mathrm{H}, \mathrm{m}), 3.05(1 \mathrm{H}, \mathrm{ddd}, J=12.0,7.2,3.6 \mathrm{~Hz}), 2.44-2.29(4 \mathrm{H}, \mathrm{m}), 1.94-1.84$ $(2 \mathrm{H}, \mathrm{m}), 1.74-1.61(2 \mathrm{H}, \mathrm{m}), 1.47(3 \mathrm{H}, \mathrm{d}, J=6.8 \mathrm{~Hz}), 1.080(3 \mathrm{H}, \mathrm{t}, J=8.0 \mathrm{~Hz}), 1.075(3 \mathrm{H}, \mathrm{t}, J=$ 7.6). ${ }^{13} \mathrm{C} \mathrm{NMR}\left(\mathrm{CDCl}_{3}, 100 \mathrm{MHz}\right): \delta$ 167.6, 143.8, 128.6, 127.0, 126.0, 79.7, 72.6, 65.3, 48.5, 28.5, 23.5, 23.4, 23.3, 20.5, 12.8, 12.6. Anal Calcd for $\mathrm{C}_{18} \mathrm{H}_{28} \mathrm{~N}_{2} \mathrm{O}_{2}$ : C, 71.02; H, 9.27; N, 9.20. Found C, 70.87; H, 9.41; N, 9.27. Optical Rotation: $[\alpha]^{25}+8.2^{\circ}$ (c 1.0, $\left.\mathrm{CHCl}_{3}\right)$.

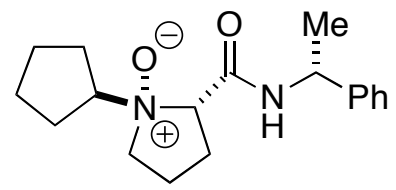

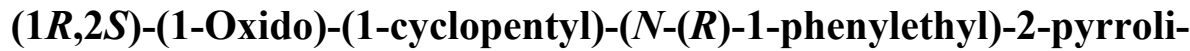 dinecarboxamide (3q). \\ Step 1: Palladium on Carbon ( $10 \%$ by wt., dry, $0.23 \mathrm{~g})$ was added} to a dry $100 \mathrm{~mL}$ flask and flushed with $\mathrm{N}_{2}$. Methanol $(40 \mathrm{~mL})$ was then slowly added. To this suspension was charged $(S)$-proline $(2.3 \mathrm{~g}, 20 \mathrm{mmol})$ and cyclopentanone $(2.0 \mathrm{~mL}, 22 \mathrm{mmol})$. The reaction was placed under an atmosphere $\mathrm{H}_{2}$ (balloon) for $16 \mathrm{~h}$. At this time the reaction was purged with $\mathrm{N}_{2}$ and filtered through celite. Removal of the methanol under reduced pressure led to a pale yellow solid which was collected and washed with diethyl ether ( $3 \mathrm{x}$ $25 \mathrm{~mL})$ to yield the unpurified acid (2.6 g, 71\% unpurified yield) as an off white solid.

Step 2: The unpurified acid $(1.5 \mathrm{~g}, 8.0 \mathrm{mmol})$ was then dissolved in DMF (40 $\mathrm{mL})$ and THF $(30 \mathrm{~mL})$. (R)-1-Phenylethylamine $(1.0 \mathrm{~mL}, 8.0 \mathrm{mmol})$ and $\mathrm{K}_{2} \mathrm{CO}_{3}$ $(1.7 \mathrm{~g}, 12 \mathrm{mmol})$ were then added and the mixture was allowed to stir for 30 minutes to dissolve the acid. TBTU (3.1 g, $9.6 \mathrm{mmol})$ and HOBt $(1.5 \mathrm{~g}, 9.6$ mmol) were then added and the reaction was allowed to stir for $2 \mathrm{~h}$ after which time water $(50 \mathrm{~mL})$ was added. The reaction was extracted with ethyl acetate $(2 \times 50 \mathrm{~mL})$. The organic layer was washed with $1 \%$ aqueous sodium carbonate $(5 \times$ x $50 \mathrm{~mL})$ and with brine $(1 \times$ x $25 \mathrm{~mL})$, dried over 
anhydrous $\mathrm{Na}_{2} \mathrm{SO}_{4}$, filtered, and concentrated under reduced pressure to yield unpurified amide (0.76 g, 33\% unpurified yield).

Step 3: The unpurified amide $(0.76 \mathrm{~g}, 2.6 \mathrm{mmol})$ was then dissolved in methylene chloride $(40 \mathrm{~mL})$. Potassium carbonate $(0.73 \mathrm{~g}, 5.3 \mathrm{mmol})$ was then added and the reaction was cooled to $-78{ }^{\circ} \mathrm{C}$. $m$-CPBA $(0.61 \mathrm{~g}$ of $75 \%$ pure, net $0.46 \mathrm{~g}, 2.6$ mmol) was then added, and the reaction was allowed to stir at -78 ${ }^{\circ} \mathrm{C}$ for $3 \mathrm{~h}$. At this time, the reaction was allowed to warm to room temperature. After stirring for 2 $\mathrm{h}$ at room temperature, methylene chloride $(50 \mathrm{~mL})$ was added to dilute the reaction and celite $(500 \mathrm{mg})$ was added to aid filtration. The reaction was then filtered, and the methylene chloride was removed under reduced pressure. The resulting oil was purified by silica gel chromatography $\left(97: 3 \mathrm{CH}_{2} \mathrm{Cl}_{2}: \mathrm{MeOH}\right)$ to yield pure $\mathbf{3} \mathbf{q}$ as a white solid (514 mg, 15\% yield over 3 steps). M.P. 86-88 ${ }^{\circ} \mathrm{C}$. IR (neat, thin film): 2962 (m), 2867 (w), 1665 (s), 1539 (s), 1495 (w), $1445(\mathrm{w}), 872(\mathrm{w}), 771(\mathrm{w}), 702(\mathrm{~m}), 545(\mathrm{w}) \mathrm{cm}^{-}$ ${ }^{1} .{ }^{1} \mathrm{H} \mathrm{NMR}\left(\mathrm{CDCl}_{3}, 400 \mathrm{MHz}\right): \delta 11.67(1 \mathrm{H}, \mathrm{d}, J=7.6 \mathrm{~Hz}), 7.31-7.20(5 \mathrm{H}, \mathrm{m}), 5.08(1 \mathrm{H}, \mathrm{p}, J=7.2$ $\mathrm{Hz}), 3.61-3.55(2 \mathrm{H}, \mathrm{m}), 3.44(1 \mathrm{H}, \mathrm{t}, J=10.4 \mathrm{~Hz}), 3.30(1 \mathrm{H}, \mathrm{q}, J=9.6 \mathrm{~Hz}), 2.40-2.35(3 \mathrm{H}, \mathrm{m}), 2.22-$ $2.14(3 \mathrm{H}, \mathrm{m}), 1.91-1.82(3 \mathrm{H}, \mathrm{m}), 1.67-1.57(3 \mathrm{H}, \mathrm{m}), 1.48(3 \mathrm{H}, \mathrm{d}, J=6.8 \mathrm{~Hz}) .{ }^{13} \mathrm{C} \mathrm{NMR}\left(\mathrm{CDCl}_{3}\right.$, $100 \mathrm{MHz}): \delta 167.8,143.8,128.5,126.8,125.9,78.3,75.4,69.4,48.5,29.4,27.3,27.1,24.3,23.6$, 23.0, 20.7. Anal Calcd for $\mathrm{C}_{18} \mathrm{H}_{26} \mathrm{~N}_{2} \mathrm{O}_{2}$ : C, 71.49; H, 8.67; N, 9.26. Found C, 71.25; H, 8.80; $\mathrm{N}$, 9.12. Optical Rotation: $[\alpha]_{\mathrm{D}}^{25}-5.4^{\circ}\left(\mathrm{c} 1.0, \mathrm{CHCl}_{3}\right)$.

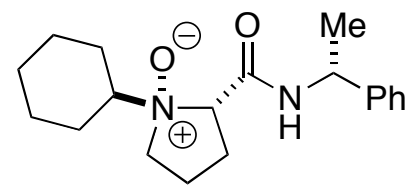

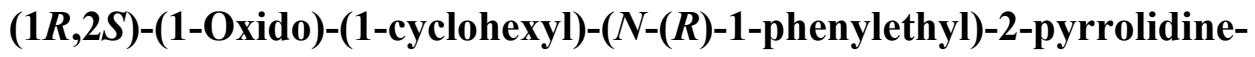
carboxamide (3r).

Step 1: Palladium on Carbon (10\% by wt., dry, $0.50 \mathrm{~g})$ was added to a dry $500 \mathrm{~mL}$ flask and flushed with $\mathrm{N}_{2}$. Methanol $(200 \mathrm{~mL})$ was then slowly added. To this suspension was charged $(S)$-proline $(11.5 \mathrm{~g}, 100 \mathrm{mmol})$ and cyclohexanone (11.4 mL, $110 \mathrm{mmol})$. The reaction flask was purged with $\mathrm{H}_{2}$ and was kept under a balloon of $\mathrm{H}_{2}$ for $16 \mathrm{~h}$. At this time the reaction was purged with $\mathrm{N}_{2}$ and filtered through celite. Removal of the methanol under reduced pressure led to a pale yellow solid which was collected and washed with diethyl ether ( $3 \times 75 \mathrm{~mL})$ to yield the unpurified acid $(17.4 \mathrm{~g}, 88 \%$ unpurified yield) as an off white solid.

Step 2: The unpurified acid (1.55 g, $7.90 \mathrm{mmol})$ was dissolved in DMF (16 $\mathrm{mL})$ and THF $(12 \mathrm{~mL})$. (R)-1-Phenylethylamine $(1.0 \mathrm{~mL}, 7.9 \mathrm{mmol})$ and $\mathrm{K}_{2} \mathrm{CO}_{3}$ $(1.63 \mathrm{~g}, 11.8 \mathrm{mmol})$ were then added and the mixture was allowed to stir for 30 minutes to dissolve the acid. TBTU $(2.8 \mathrm{~g}, 8.6 \mathrm{mmol})$ and HOBt $(1.32 \mathrm{~g}, 8.60$ mmol) were then added and the reaction was allowed to stir for $2 \mathrm{~h}$ after which time water $(30 \mathrm{~mL})$ was added. The reaction was extracted with ethyl acetate $(3 \mathrm{x} 40 \mathrm{~mL})$. The organic layer was washed with $1 \%$ aqueous sodium carbonate $(5 \times \mathrm{x} 30 \mathrm{~mL})$ and with brine $(1 \mathrm{x} 30 \mathrm{~mL})$, dried over anhydrous $\mathrm{Na}_{2} \mathrm{SO}_{4}$, filtered, and concentrated under reduced pressure to yield unpurified amide (2.12 g, $90 \%$ unpurified yield).

Step 3: The unpurified amide $(2.12 \mathrm{~g}, 7.10 \mathrm{mmol})$ was then dissolved in methylene chloride $(50 \mathrm{~mL})$. Potassium carbonate $(2.00 \mathrm{~g}, 14.1 \mathrm{mmol})$ was then added and the reaction was cooled to $-78{ }^{\circ} \mathrm{C}$. $m$-CPBA $(1.60 \mathrm{~g}$ of $75 \%$ pure, net $1.22 \mathrm{~g}, 7.10 \mathrm{mmol})$ was then added, and the reaction was allowed to stir at $-78^{\circ} \mathrm{C}$ for $2 \mathrm{~h}$. At this time, the reaction was allowed to warm to room temperature. After stirring for $2 \mathrm{~h}$ at room temperature, methylene chloride $(50 \mathrm{~mL}) \mathrm{was}$ added to dilute the reaction and celite $(2 \mathrm{~g})$ was added to aid filtration. The reaction was then filtered, and the methylene chloride was removed under reduced pressure. The resulting solid was 
collected and stirred with diethyl ether $(50 \mathrm{~mL})$ for 5 minutes. The resulting solids were collected and washed with diethyl ether $(3 \times 50 \mathrm{~mL})$ to yield pure $3 \mathrm{r}$ as a white powder $(1.67 \mathrm{~g}, 60 \%$ yield over 3 steps). Crystals suitable for $\mathrm{X}$-ray analysis were grown from $\mathrm{CH}_{2} \mathrm{Cl}_{2} /$ hexanes. M.P. 121-122 ${ }^{\circ} \mathrm{C}$. IR (neat, thin film): 2943 (br), 2861 (m), 1665 (s), 1552 (s), 1501 (w), 1451 (m), 866 (w), 759 $(\mathrm{m}), 702(\mathrm{~m}), 526(\mathrm{w}) \mathrm{cm}^{-1} .{ }^{1} \mathrm{H}$ NMR $\left(\mathrm{CDCl}_{3}, 400 \mathrm{MHz}\right): \delta 11.81(1 \mathrm{H}, \mathrm{d}, J=6.8 \mathrm{~Hz}), 7.31-7.19$ $(5 \mathrm{H}, \mathrm{m}), 5.10(1 \mathrm{H}, \mathrm{p}, J=7.2 \mathrm{~Hz}), 3.65(1 \mathrm{H}, \mathrm{br}), 3.42(1 \mathrm{H}, \mathrm{dd}, J=6.0,4.8 \mathrm{~Hz}), 3.29(1 \mathrm{H}, \mathrm{dd}, J=$ $10.8,8.8 \mathrm{~Hz}), 3.14(1 \mathrm{H}, \mathrm{tt}, J=12.0,3.6 \mathrm{~Hz}), 2.40-2.37(4 \mathrm{H}, \mathrm{m}), 2.14(1 \mathrm{H}, \mathrm{d}, J=12.0 \mathrm{~Hz}), 1.93-$ $1.90(3 \mathrm{H}, \mathrm{m}), 1.71-1.58(3 \mathrm{H}, \mathrm{m}), 1.48(3 \mathrm{H}, \mathrm{d}, J=6.8 \mathrm{~Hz}), 1.35-1.12(3 \mathrm{H}, \mathrm{m}) .{ }^{13} \mathrm{C} \mathrm{NMR}\left(\mathrm{CDCl}_{3}\right.$, $100 \mathrm{MHz}): \delta 168.0,143.8,128.5,126.8,125.9,77.5,76.3,72.7,66.6,48.5,28.7,27.9,25.7,25.6$, 25.3, 23.1, 20.4. Anal Calcd for $\mathrm{C}_{19} \mathrm{H}_{28} \mathrm{~N}_{2} \mathrm{O}_{2}$ : C, 72.12; H, 8.92; N, 8.88. Found C, 71.93; H, 9.20; $\mathrm{N}, 8.88$. Optical Rotation: $[\alpha]^{25}-1.3^{\circ}$ (c 3.0, $\mathrm{CHCl}_{3}$ ).

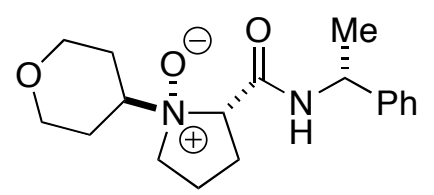

(1R,2S)-(1-Oxido)-(1-tetrahydro-pyran-4-yl)-( $N$-(R)-1-phenylethyl)2-pyrrolidinecarboxamide (3s). The procedure for $\mathbf{3 q}$ was followed, only tetrahydro-pyran-4-one $(0.99 \mathrm{~g}, 5.0 \mathrm{mmol})$ was substituted for cyclopentanone in step 1 . Unpurified $\mathbf{3 s}$ was purified by silica gel chromatography $\left(95: 5 \mathrm{CH}_{2} \mathrm{Cl}_{2}: \mathrm{MeOH}\right)$ to yield pure $3 \mathrm{~s}$ as a white solid (580 $\mathrm{mg}, 66 \%$ yield over 3 steps). M.P. $135-137^{\circ}$ C. IR (neat, thin film): 2964 (br), 2852 (br), 1666 (s), 1549 (s), 1499 (w), 1462 (w), 1257(w), 1164 (w), 1095 (w), $1027(\mathrm{w}), 990(\mathrm{w}), 878(2), 773(\mathrm{w}), 711(\mathrm{~m}) \mathrm{cm}^{-1} \cdot{ }^{1} \mathrm{H}$ NMR $\left(\mathrm{CDCl}_{3}, 400 \mathrm{MHz}\right): \delta 11.60(1 \mathrm{H}, \mathrm{d}, J=8.0 \mathrm{~Hz}), 7.31-7.21(5 \mathrm{H}, \mathrm{m}), 5.08(1 \mathrm{H}, \mathrm{p}, J=6.8 \mathrm{~Hz})$, 4.13-4.10 (2H, m), $3.64(1 \mathrm{H}, \mathrm{t}, J=8.8 \mathrm{~Hz}), 3.45-3.24(6 \mathrm{H}, \mathrm{m}), 2.42-2.36(3 \mathrm{H}, \mathrm{m}), 2.24(1 \mathrm{H}, \mathrm{d}, J=$ $12.0 \mathrm{~Hz}), 2.16-1.91(5 \mathrm{H}, \mathrm{m}), 1.49(1 \mathrm{H}, \mathrm{t}, J=6.4 \mathrm{~Hz}) .{ }^{13} \mathrm{C} \mathrm{NMR}\left(\mathrm{CDCl}_{3}, 100 \mathrm{MHz}\right): \delta 167.6,143.7$, $128.7,127.0,126.0,73.2,73.1,67.1,66.6,66.5,48.7,28.7,28.2,28.1,23.2,20.4$. Anal Calcd for $\mathrm{C}_{18} \mathrm{H}_{26} \mathrm{~N}_{2} \mathrm{O}_{3}$ : C, 67.90; H, 8.23; N, 8.80. Found C, 68.02; H, 8.43; N, 8.79. Optical Rotation: $[\alpha]^{25}$ $+5.8^{\circ}\left(\mathrm{c} 1.0, \mathrm{CHCl}_{3}\right)$.

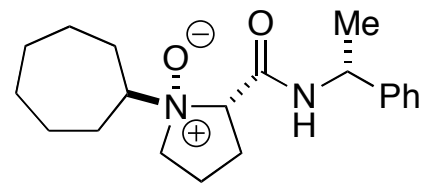

(1R,2S)-(1-Oxido)-(1-cycloheptyl)-( $N$-(R)-1-phenylethyl)-2-pyrrolidinecarboxamide (3t). The procedure for $\mathbf{3 q}$ was followed, only cycloheptanone $(2.60 \mathrm{~mL}, 22.0 \mathrm{mmol})$ was substituted for cyclopentanone in step 1. Unpurified 3t was recrystalized from $\mathrm{CH}_{2} \mathrm{Cl}_{2}$ / hexanes to yield pure $3 \mathrm{t}$ ( $894 \mathrm{mg}, 25 \%$ yield for 3 steps) as an off white solid. M.P. $105-107{ }^{\circ} \mathrm{C}$. IR (neat, thin film): 2924 (m), 2855 (w), 1671 (s), 1539 (s), 1451 (m), 759 (w), 656 (m), 532 (w) $\mathrm{cm}^{-1} .{ }^{1} \mathrm{H}$ NMR $\left(\mathrm{CDCl}_{3}, 400 \mathrm{MHz}\right): \delta 11.94(1 \mathrm{H}, \mathrm{d}, J=7.6 \mathrm{~Hz}), 7.32-7.21(5 \mathrm{H}, \mathrm{m}), 5.13(1 \mathrm{H}, \mathrm{p}, J=$ $7.6 \mathrm{~Hz}), 3.75(1 \mathrm{H}, \mathrm{dd}, J=11.2,8.0 \mathrm{~Hz}), 3.55(1 \mathrm{H}, \mathrm{tt}, J=8.8,3.6 \mathrm{~Hz}), 3.41-3.25(2 \mathrm{H}, \mathrm{m}), 2.51-2.30$ $(4 \mathrm{H}, \mathrm{m}), 2.11-2.06(1 \mathrm{H}, \mathrm{m}), 1.90-1.52(10 \mathrm{H}, \mathrm{m}), 1.49(3 \mathrm{H}, \mathrm{d}, J=7.2 \mathrm{~Hz}), 1.43-1.32(1 \mathrm{H}, \mathrm{m}) .{ }^{13} \mathrm{C}$ NMR $\left(\mathrm{CDCl}_{3}, 100 \mathrm{MHz}\right): \delta 167.6,143.8,128.6,126.9,126.0,77.6,72.3,64.8,48.5,29.7,28.8$, 28.6, 28.4, 27.9, 25.9, 25.1, 23.5, 20.5. Anal Calcd for $\mathrm{C}_{20} \mathrm{H}_{30} \mathrm{~N}_{2} \mathrm{O}_{2}$ : C, 72.69; H, 9.15; N, 8.48. Found C, 72.90; H, 9.15; N, 8.24. Optical Rotation: $[\alpha]^{25}-5.0^{\circ}\left(\mathrm{c} 1.0, \mathrm{CHCl}_{3}\right)$.

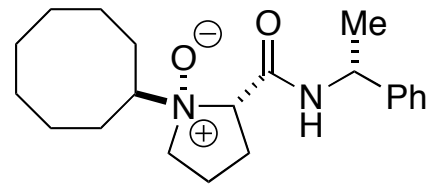

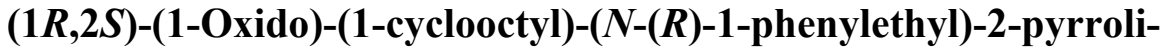
dinecarboxamide $(3 \mathbf{u})$. The procedure for $\mathbf{3 q}$ was followed, only cyclooctanone $(2.80 \mathrm{~g}, 22.0 \mathrm{mmol})$ was substituted for cyclopentanone in step 1. Unpurified $3 \mathbf{u}$ was recrystalized from $\mathrm{CH}_{2} \mathrm{Cl}_{2} /$ hexanes to yield pure 3u (897 mg, 21\% yield for 3 steps) as a white solid. M.P. $117-119{ }^{\circ} \mathrm{C}$. IR (neat, thin film): 2924 (m), 2861 (w), 1659 (s), 1545 (m), 1495 (w), 1457 (w), 904 (w), 771 (w), $702(\mathrm{~m}), 513(\mathrm{w}) \mathrm{cm}^{-1} .{ }^{1} \mathrm{H}$ NMR $\left(\mathrm{CDCl}_{3}, 400 \mathrm{MHz}\right): \delta 11.88(1 \mathrm{H}, \mathrm{d}, J=8.0 \mathrm{~Hz}), 7.33-7.20(5 \mathrm{H}, \mathrm{m})$, $5.12(1 \mathrm{H}, \mathrm{p}, J=7.6 \mathrm{~Hz}), 3.75(1 \mathrm{H}, \mathrm{dd}, J=11.2,7.6 \mathrm{~Hz}), 3.44-3.27(3 \mathrm{H}, \mathrm{m}), 2.56-2.20(6 \mathrm{H}, \mathrm{m})$, 
1.91-1.56 (12H, m), $1.49(3 \mathrm{H}, \mathrm{d}, J=6.8 \mathrm{~Hz}) .{ }^{13} \mathrm{C} \mathrm{NMR}\left(\mathrm{CDCl}_{3}, 100 \mathrm{MHz}\right): \delta 167.7,143.8,128.6$, 127.0, 126.0, 76.3, 72.5, 64.9, 48.6, 29.0, 28.8, 28.5, 27.0, 26.5, 26.4, 26.2, 25.8, 23.5, 20.5. Anal Calcd for $\mathrm{C}_{21} \mathrm{H}_{32} \mathrm{~N}_{2} \mathrm{O}_{2}$ : C, 73.22; H, 9.36; N, 8.13. Found C, 72.97; H, 9.42; N, 8.42. Optical Rotation: $[\alpha]^{25}-2.2^{\circ}$ (c $\left.1.0, \mathrm{CHCl}_{3}\right)$.

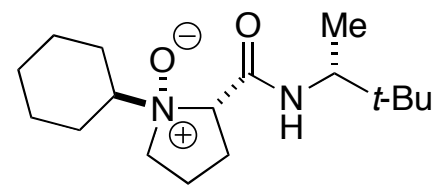

$(1 R, 2 S)-(1-O x i d o)-(1-c y c l o h e x y l)-(N-(R)-1,2,2-t r i m e t h y l-p r o p y l))-2-$ pyrrolidinecarboxamide $(3 \mathrm{v})$. The procedure (modified for scale) for $3 \mathbf{r}$ was followed, only $(R)$-3,3-dimethyl-2-butylamine $(1.30 \mathrm{~mL}, 10.0$ $\mathrm{mmol}$ ) was substituted for $(R)-1$-phenylethylamine in step 2. Unpurified 3v was recrystalized from $\mathrm{CH}_{2} \mathrm{Cl}_{2} /$ Hexanes to yield pure $\mathbf{3 v}$ as an off-white solid $(1.01 \mathrm{~g}, 50 \%$ yield over 3 steps). M.P. 178-180 ${ }^{\circ} \mathrm{C}$. IR (neat, thin film): 2955 (s), 2861 (m), 1659 (s), $1558(\mathrm{~m})$, $1451(\mathrm{w}), 1363$ (w), 1136 (w), $891(\mathrm{w}), 746(\mathrm{w}), 664(\mathrm{w}) \mathrm{cm}^{-1} .{ }^{1} \mathrm{H} \mathrm{NMR}\left(\mathrm{CDCl}_{3}, 400 \mathrm{MHz}\right): \delta$ $11.35(1 \mathrm{H}, \mathrm{d}, J=7.6 \mathrm{~Hz}), 3.77(1 \mathrm{H}, \mathrm{dq}, J=9.6,6.8 \mathrm{~Hz}), 3.61(1 \mathrm{H}, \mathrm{t}, J=9.2 \mathrm{~Hz}), 3.40(1 \mathrm{H}, \mathrm{tt}, J=$ 8.4, 3.2 Hz), $3.26(1 \mathrm{H}, \mathrm{q}, J=9.6 \mathrm{~Hz}), 3.07(1 \mathrm{H}, \mathrm{tt}, J=11.6,3.6 \mathrm{~Hz}), 2.46-2.31(4 \mathrm{H}, \mathrm{m}), 2.09(1 \mathrm{H}$, $\mathrm{dt}, J=12.0,2.8 \mathrm{~Hz}), 1.92-1.86(3 \mathrm{H}, \mathrm{m}), 1.68-1.50(3 \mathrm{H}, \mathrm{m}), 1.31-1.11(3 \mathrm{H}, \mathrm{m}), 1.06(3 \mathrm{H}, \mathrm{d}, J=6.6$ $\mathrm{Hz}), 0.90(9 \mathrm{H}, \mathrm{s}) .{ }^{13} \mathrm{C} \mathrm{NMR}\left(\mathrm{CDCl}_{3}, 100 \mathrm{MHz}\right): \delta 168.0,76.3,72.9,66.7,52.7,34.6,29.0,27.9$, 27.8, 26.6, 25.7, 25.6, 25.3, 20.4, 15.8. Anal. Calcd for $\mathrm{C}_{17} \mathrm{H}_{32} \mathrm{~N}_{2} \mathrm{O}_{3}$ : C, 68.88; H, 10.88; N, 9.45. Found C, 68.68; H, 11.09; N, 9.38. Optical Rotation: $[\alpha]_{\mathrm{D}}^{25}-49^{\circ}$ (c 1.0, $\mathrm{CHCl}_{3}$ ).

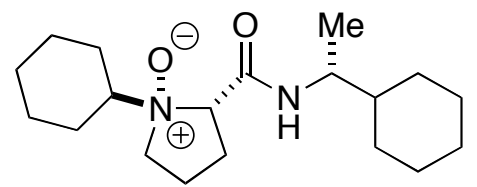

$(1 R, 2 S)-(1-O x i d o)-(1-c y c l o h e x y l)-(N-(R)-1-c y c l o h e x y l-e t h y l)-2-p y r-$ rolidinecarboxamide (3w). The procedure for $3 \mathbf{r}$ was followed, only $(R)$-1-cyclohexylethylamine $(0.73 \mathrm{~mL}, 5.0 \mathrm{mmol})$ was substituted for $(R)$-1-phenylethylamine, with adjustments for scale, in step 2. Unpurified 3w purified by silica gel chromatography $\left(95: 5 \mathrm{CH}_{2} \mathrm{Cl}_{2}: \mathrm{MeOH}\right)$ to yield pure $\mathbf{3 w}$ as pale yellow solid ( $0.81 \mathrm{~g}, 44 \%$ yield over 3 steps). M.P. $149-151^{\circ} \mathrm{C}$. IR (neat, thin film): 2924 (s), 2855 (m), 1671 (s), 1546 (m), 1451 (m), 904 (w), 866 (w), 759 (w), $671(\mathrm{w}), 526$ (w) $\mathrm{cm}^{-1} .{ }^{1} \mathrm{H}$ NMR $\left(\mathrm{CDCl}_{3}, 400 \mathrm{MHz}\right): \delta 11.15(1 \mathrm{H}, \mathrm{d}, J=8.0 \mathrm{~Hz}), 3.80(1 \mathrm{H}, \mathrm{ddq}, J=13.2,9.6,6.8 \mathrm{~Hz}), 3.61(1 \mathrm{H}, \mathrm{t}, J$ $=8.8 \mathrm{~Hz}), 3.40(1 \mathrm{H}, \mathrm{t}, J=8.4 \mathrm{~Hz}), 3.27(1 \mathrm{H}, \mathrm{q}, J=9.6 \mathrm{~Hz}), 3.09(1 \mathrm{H}, \mathrm{t}, J=12.0 \mathrm{~Hz}), 2.48-2.33$ $(4 \mathrm{H}, \mathrm{m}), 2.11(1 \mathrm{H}, \mathrm{d}, J=8.0 \mathrm{~Hz}), 1.91-1.87(2 \mathrm{H}, \mathrm{m}), 1.79-1.50(8 \mathrm{H}, \mathrm{m}), 1.36-0.93(10 \mathrm{H}, \mathrm{m}), 1.12$ $(3 \mathrm{H}, \mathrm{d}, J=6.4 \mathrm{~Hz}) .{ }^{13} \mathrm{C} \mathrm{NMR}\left(\mathrm{CDCl}_{3}, 100 \mathrm{MHz}\right): \delta 168.1,76.2,72.8,66.5,49.1,43.3,29.8,29.1$, 29.0, 27.9, 27.8, 26.7, 26.5, 26.5, 25.7, 25.6, 25.3, 20.3, 18.0. Anal Calcd for $\mathrm{C}_{19} \mathrm{H}_{34} \mathrm{~N}_{2} \mathrm{O}_{3}: \mathrm{C}, 70.76$; $\mathrm{H}, 10.63 ; \mathrm{N}, 8.69$. Found C, 70.69; H, 10.90; N, 8.40. Optical Rotation: $[\alpha]_{\mathrm{D}}^{25}-38^{\circ}$ (c 1.0, $\left.\mathrm{CHCl}_{3}\right)$.

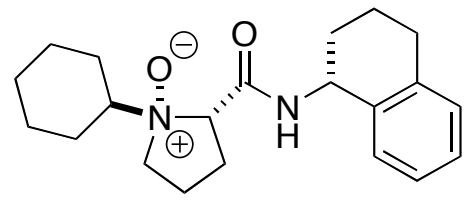

$(1 R, 2 S)-(1-O x i d o)-(1-c y c l o h e x y)-(N-(R)-1-(1,2,3,4-t e t r a h y d r o-$ naphthyl))-2-pyrrolidinecarboxamide $(\mathbf{3 x})$. The procedure for $\mathbf{3 r}$ was followed, only $(R)-1,2,3,4$-tetrahydro-1-naphthylamine $(0.74 \mathrm{~mL}$, $5.0 \mathrm{mmol}$ ) was substituted for $(R)$-1-Phenylethylamine, with adjustments for scale, in step 2. Unpurified $\mathbf{3 x}$ was recrystalized from $\mathrm{CH}_{2} \mathrm{Cl}_{2}$ to yield pure $3 \mathbf{x}$ as an off-white solid (1.12 g, 58\% yield over 3 steps). M.P. $172-174{ }^{\circ} \mathrm{C}$. IR (neat, thin film): 2936 (m), 2855 (w), 1659 (s), 1539 (s), 1489 (w), $1451(\mathrm{~m}), 1338(\mathrm{w}), 872(\mathrm{w})$, $752(\mathrm{~m}), 664(\mathrm{w}), 532(\mathrm{~m}) \mathrm{cm}^{-1} .{ }^{1} \mathrm{H} \mathrm{NMR}\left(\mathrm{CDCl}_{3}, 400 \mathrm{MHz}\right): \delta 11.56(1 \mathrm{H}, \mathrm{d}, J=8.8 \mathrm{~Hz}), 7.30-7.27$ $(2 \mathrm{H}, \mathrm{m}), 7.19-7.08(2 \mathrm{H}, \mathrm{m}), 5.25-5.19(1 \mathrm{H}, \mathrm{m}), 3.75(1 \mathrm{H}, \mathrm{t}, J=9.6 \mathrm{~Hz}), 3.42(1 \mathrm{H}, \mathrm{dt}, J=11.2,2.8$ $\mathrm{Hz}), 3.32(1 \mathrm{H}, \mathrm{q}, J=10.0 \mathrm{~Hz}), 3.16(1 \mathrm{H}, \mathrm{tt}, J=11.2,3.2 \mathrm{~Hz}), 2.89-2.76(2 \mathrm{H}, \mathrm{m}), 2.56-2.38(4 \mathrm{H}, \mathrm{m})$, 2.19-2.11 (2H, m), 1.99-1.79 (6H, m), 1.72-1.57 (3H, m), 1.38-1.15 (3H, m). ${ }^{13} \mathrm{C} \mathrm{NMR}\left(\mathrm{CD}_{3} \mathrm{OD}\right.$, $100 \mathrm{MHz}): \delta 169.2,137.5,136.6,129.3,128.3,127.5,126.3,76.6,73.5,66.7,47.7,31.2,29.4,28.9$, 
28.3, 27.9, 25.8, 25.7, 25.5, 20.7, 20.2. Anal Calcd for $\mathrm{C}_{21} \mathrm{H}_{30} \mathrm{~N}_{2} \mathrm{O}_{3}$ : C, 73.65; H, 8.83; N, 8.18. Found C, 73.80; H, 9.05; N, 7.93. Optical Rotation: $[\alpha]_{\mathrm{D}}^{25}-21^{\circ}$ (c 1.0, $\left.\mathrm{CHCl}_{3}\right)$.

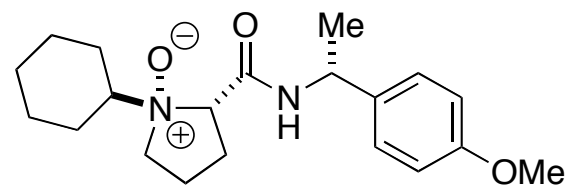

(1R,2S)-(1-Oxido)-(1-cyclohexyl)-( $N$-(R)-1-(4-methoxy-phenyl-ethyl))-2-pyrrolidinecarboxamide (3y). The procedure for 3r was followed, only $(R)$-1-(4-methoxyphenyl)ethylamine (364 $\mathrm{mg}, 2.5 \mathrm{mmol}$ ) was substituted for $(R)$-1-Phenylethylamine in step 2. Unpurified $\mathbf{3 y}$ was collected and triturated with diethyl ether $(20 \mathrm{~mL})$. The solid was then collected and washed with diethyl ether $(3 \times 20 \mathrm{~mL})$ to yield $\mathbf{3 y}$ as an off-white solid (594 mg, 61\% yield over 3 steps). M.P. $71-73{ }^{\circ} \mathrm{C}$. IR (neat, thin film): 2933 (br), 2858 (m), 1660 (s), 1611 (w), 1542 (m), 117 (s), 1449 (w), 1294 (w), 1250 (s), $1182(\mathrm{~m}), 1027(\mathrm{w}), 835(\mathrm{~m}), 748(\mathrm{w}), 531(\mathrm{w}) \mathrm{cm}^{-}$ 1. ${ }^{1} \mathrm{H} \mathrm{NMR}\left(\mathrm{CDCl}_{3}, 400 \mathrm{MHz}\right): \delta 11.71(1 \mathrm{H}, \mathrm{d}, J=7.6 \mathrm{~Hz}), 7.23(2 \mathrm{H}, \mathrm{d}, J=8.4 \mathrm{~Hz}), 6.83(2 \mathrm{H}, \mathrm{d}, J$ $=8.8 \mathrm{~Hz}), 5.04(1 \mathrm{H}, \mathrm{p}, J=7.2 \mathrm{~Hz}), 3.77(3 \mathrm{H}, \mathrm{s}), 3.63(1 \mathrm{H}, \mathrm{t}, J=8.8 \mathrm{~Hz}), 3.40(1 \mathrm{H}, \mathrm{dt}, J=6.0,1.6$ $\mathrm{Hz}), 3.26(1 \mathrm{H}, \mathrm{dt}, J=10.4,8.8 \mathrm{~Hz}), 3.13(1 \mathrm{H}, \mathrm{tt}, J=12.0,3.2 \mathrm{~Hz}), 2.41-2.33(4 \mathrm{H}, \mathrm{m}), 2.13(1 \mathrm{H}, \mathrm{d}$, $J=12.4 \mathrm{~Hz}), 1.92-1.86(3 \mathrm{H}, \mathrm{m}), 1.71-1.57(3 \mathrm{H}, \mathrm{m}), 1.45(3 \mathrm{H}, \mathrm{d}, J=6.8 \mathrm{~Hz}), 1.38-1.10(3 \mathrm{H}, \mathrm{m}) .{ }^{13} \mathrm{C}$ $\mathrm{NMR}\left(\mathrm{CDCl}_{3}, 100 \mathrm{MHz}\right): \delta 167.9,158.5,136.2,127.1,114.0,76.4,72.9,66.7,55.5,48.0,28.8$, 25.8, 25.7, 25.4, 23.1, 20.5. Anal Calcd for $\mathrm{C}_{20} \mathrm{H}_{30} \mathrm{~N}_{2} \mathrm{O}_{3}: \mathrm{C}, 69.33 ; \mathrm{H}, 8.73 ; \mathrm{N}, 8.09$. Found $\mathrm{C}$, 69.21; H, 9.00; N, 8.12. Optical Rotation: $[\alpha]_{\mathrm{D}}^{25}+7.8^{\circ}\left(\mathrm{c} 1.0, \mathrm{CHCl}_{3}\right)$.

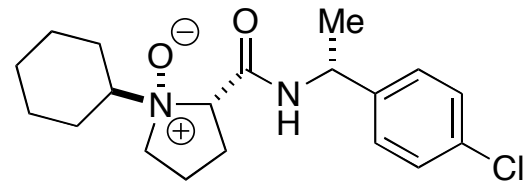

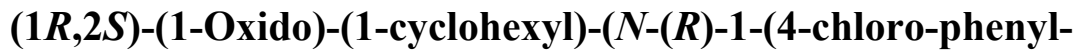
ethyl))-2-pyrrolidinecarboxamide (3z). The procedure for $\mathbf{3 r}$ was followed, only $(R)-1-(4-C h l o r o p h e n y l) e t h y l a m i n e ~(0.34 \mathrm{~mL}$,

$2.5 \mathrm{mmol})$ was substituted for $(R)$-1-Phenylethylamine, with adjustments for scale, in step 2. Unpurified $\mathbf{3 z}$ was collected and washed with diethyl ether ( $3 \times 20$ $\mathrm{mL}$ ) to yield $\mathbf{3 z}$ as an off-white solid (371 mg, 37\% yield over 3 steps). M.P. 91-93 ${ }^{\circ} \mathrm{C}$. IR (neat, thin film): 2939 (br), 2864 (w), 1666 (s), 1542 (s), 1493 (m), 1449 (w), 1344 (w), 1095 (w), 1015 (w), $829(\mathrm{~m}), 754(\mathrm{w}), 543(\mathrm{~m}) \mathrm{cm}^{-1} .{ }^{1} \mathrm{H} \mathrm{NMR}\left(\mathrm{CDCl}_{3}, 400 \mathrm{MHz}\right): \delta 11.89(1 \mathrm{H}, \mathrm{d}, J=7.2 \mathrm{~Hz}), 7.26$ $(2 \mathrm{H}, \mathrm{d}, J=2.0 \mathrm{~Hz}), 7.26(2 \mathrm{H}, \mathrm{d}, J=2.0 \mathrm{~Hz}), 5.06(1 \mathrm{H}, \mathrm{p}, J=7.2 \mathrm{~Hz}), 3.65(1 \mathrm{H}, \mathrm{t}, J=9.6 \mathrm{~Hz}), 3.43$ $(1 \mathrm{H}, \mathrm{dt}, J=8.0,2.4 \mathrm{~Hz}), 3.32-3.25(1 \mathrm{H}, \mathrm{m}), 3.14(1 \mathrm{H}, \mathrm{tt}, J=12.0,4.0 \mathrm{~Hz}), 2.43-2.33(4 \mathrm{H}, \mathrm{m}), 2.14$ $(1 \mathrm{H}, \mathrm{d}, J=12.0 \mathrm{~Hz}), 1.94-1.91(3 \mathrm{H}, \mathrm{m}), 1.69-1.58(3 \mathrm{H}, \mathrm{m}), 1.46(3 \mathrm{H}, \mathrm{d}, J=7.2 \mathrm{~Hz}), 1.36-1.15(3 \mathrm{H}$, m). ${ }^{13} \mathrm{C} \mathrm{NMR}\left(\mathrm{CDCl}_{3}, 100 \mathrm{MHz}\right): \delta 168.2,142.6,132.6,128.7,127.5,76.5,72.8,66.8,48.0,28.9$, 27.9, 25.8, 25.7, 25.4, 23.1, 20.5. Anal Calcd for $\mathrm{C}_{19} \mathrm{H}_{27} \mathrm{ClN}_{2} \mathrm{O}_{2}$ : C, 65.04; $\mathrm{H}, 7.76 ; \mathrm{N}, 7.98$. Found C, 64.83; H, 7.94; N, 7.93. Optical Rotation: $[\alpha]^{25}+7.8^{\circ}\left(\mathrm{c} 1.0, \mathrm{CHCl}_{3}\right)$.

Representative procedure for the Catalytic Asymmetric Allylation of Aldehydes with Allyltrichlorosilane and Proline Based N-Oxide Ligand. In a $\mathrm{N}_{2}$ atmosphere glovebox, ligand 3r was weighed out to a dry round-bottomed flask and dissolved in 1,2-dicloroethane (DCE). The aldehyde was added and the reaction was allowed to stir for 5 minutes. Allyltrichlorosilane was then added drop wise. The reaction was sealed with a septum and Teflon tape and taken out of glovebox. The reaction was allowed to stir for 24 hours at room temperature $\left(23{ }^{\circ} \mathrm{C}\right)$. To quench, the reaction was cooled to $0{ }^{\circ} \mathrm{C}$ in an ice bath and diisopropylethylamine (DIPEA, 5 equiv. with respect to the aldehyde) was added. $3 \mathrm{M} \mathrm{NaOH}$ (aq) was then added and the reaction was vigorously stirred for $2 \mathrm{~h}$. The reaction was extracted with diethyl ether $(3 \mathrm{x})$, and the combined organic layers were washed with $10 \%$ citric acid $(1 \mathrm{x})$, brine $(1 \mathrm{x})$, dried over anhydrous $\mathrm{Na}_{2} \mathrm{SO}_{4}$

\footnotetext{
${ }^{5}$ The addition of DIPEA during workup was found to facilitate the hydrolysis of the O-Si bond. In addition, for electron-rich substrates (such as entry 5, Table 4), epimerization of the product was found when the reaction was quenched under acidic conditions.
} 
and concentrated. The products were purified by silica gel chromatography. The purified products were analyzed for enantioenrichment by chiral HPLC (Chiracel OD or AS), or GLC (Supelco Alpha, Beta, or Gamma Dex 120).<smiles>C=CC[C@H](O)c1ccccc1</smiles>

(R)-1-Phenyl-3-buten-3-ol (Entry 1, Table 4). The general procedure was followed with ligand $3 \mathbf{r}(32 \mathrm{mg}, 0.10 \mathrm{mmol})$, and benzaldehyde $(102 \mu \mathrm{L}, 1.0 \mathrm{mmol})$ in DCE (2.0 $\mathrm{mL}, 0.5 \mathrm{M}$ in substrate). Allyltrichlorosilane $(217 \mu \mathrm{L}, 1.5 \mathrm{mmol})$ was then added. The reaction was sealed and allowed to react for $24 \mathrm{~h}$. After workup, the product was purified by silica gel chromatography ( $85: 15$ hexanes:diethyl ether) to yield a pale yellow oil (128 mg, 86\% yield, 87\% ee). ${ }^{1} \mathrm{H}$ NMR $\left(\mathrm{CDCl}_{3}, 400 \mathrm{MHz}\right): \delta 7.38-7.27(5 \mathrm{H}, \mathrm{m}), 5.82(1 \mathrm{H}$, ddt, $J=17.2$, $10.0,7.2 \mathrm{~Hz}), 5.17(1 \mathrm{H}, \mathrm{dd}, J=17.2,1.2 \mathrm{~Hz}), 5.15(1 \mathrm{H}, \mathrm{dd}, J=10.4,1.2 \mathrm{~Hz}), 4.74(1 \mathrm{H}, \mathrm{dt}, J=6.4$, $2.4 \mathrm{~Hz}), 2.58-2.6(2 \mathrm{H}, \mathrm{m}), 2.06(1 \mathrm{H}, \mathrm{d}, J=2.8 \mathrm{~Hz}) .{ }^{13} \mathrm{C} \mathrm{NMR}\left(\mathrm{CDCl}_{3}, 100 \mathrm{MHz}\right): \delta 143.9,134.5$, 128.5, 127.6, 125.9, 118.5, 73.5, 44.1. Optical Rotation: $[\alpha]^{25}{ }_{\mathrm{D}}+62^{\circ}\left(\mathrm{c} 1.0, \mathrm{CHCl}_{3}\right){ }^{6}$

Optical purity is established by chiral HPLC analysish(iralcel OD (4.6 mm x $250 \mathrm{~mm})$, 99:1 hexanes:isopropanol, $0.8 \mathrm{~mL} / \mathrm{min}, \lambda=220 \mathrm{~nm}$.); chromatograms are illustrated below for a $87 \%$ ee sample:

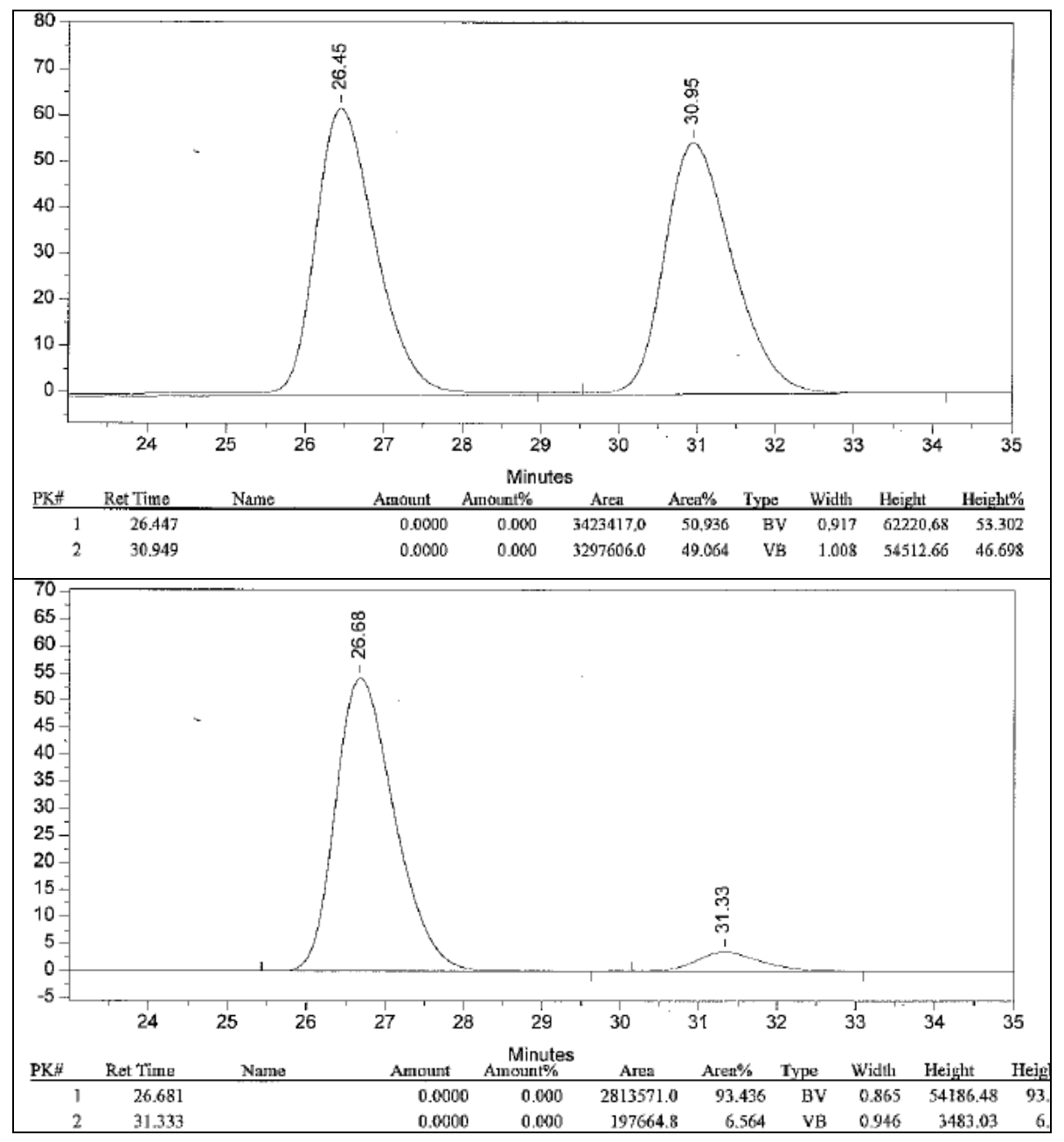

\footnotetext{
${ }^{6}$ Corresponds to $(R)$ enantiomer. See (a) Malkov, A. V.; Orsini, M.; Pernazza, D.; Muir, K. W.; Langer, V.; Meghani, P.; Kocovsky, P. Org. Lett. 2002, 4, 1047-1049. ${ }^{1} \mathrm{H}$ and ${ }^{13} \mathrm{C}$ NMR data are consistent with known product. See (b) Denmark, S. E.; Fu, J. J. Am. Chem. Soc. 2001, 123, 9488-9489.
} 
<smiles>C=CC[C@H](O)c1ccccc1Br</smiles>

(R)-1-(2-Bromophenyl)-3-buten-1-ol (Entry 2, Table 4). The general procedure was followed with ligand $3 \mathbf{r}(63 \mathrm{mg}, 0.20 \mathrm{mmol})$, and $o$-bromobenzaldehyde $(234 \mu \mathrm{L}, 2.00 \mathrm{mmol})$ in DCE $(4.0 \mathrm{~mL}, 0.5 \mathrm{M}$ in substrate). Allyltrichlorosilane $(362 \mu \mathrm{L}, 2.50 \mathrm{mmol})$ was then added. The reaction was sealed and allowed to react for $24 \mathrm{~h}$. After workup, the product was purified by silica gel chromatography (95:5 hexanes:diethyl ether) to yield a white solid (368 $\mathrm{mg}, 81 \%$ yield, 82\% ee). M.P. 45-46 ${ }^{\circ}$ C. IR (neat, thin film): 3383 (br), 3075 (w), 2980 (w), 2911 (w), 1640 (w), 1564 (w), $1470(\mathrm{~m}), 1438(\mathrm{~m}), 1199(\mathrm{w}), 1023(\mathrm{~m}), 916(\mathrm{~m}), 872(\mathrm{w}), 759$ (s), $614(\mathrm{w}) \mathrm{cm}^{-1} .{ }^{1} \mathrm{H} \mathrm{NMR}\left(\mathrm{CDCl}_{3}\right.$, $400 \mathrm{MHz}): \delta 7.56(1 \mathrm{H}, \mathrm{dd}, J=7.6,1.6 \mathrm{~Hz}), 7.52(1 \mathrm{H}, \mathrm{dd}, J=8.0,1.2 \mathrm{~Hz}), 7.34(1 \mathrm{H}, \mathrm{dt}, J=7.2,0.8$ $\mathrm{Hz}), 7.13(1 \mathrm{H}, \mathrm{dt}, J=7.6,2.0 \mathrm{~Hz}), 5.88(1 \mathrm{H}$, dddd, $J=16.8,10.0,7.6,6.4 \mathrm{~Hz}), 5.20(1 \mathrm{H}, \mathrm{d}, J=$ $17.2 \mathrm{~Hz}), 5.18(1 \mathrm{H}, \mathrm{d}, J=10.0 \mathrm{~Hz}), 5.11(1 \mathrm{H}, \mathrm{dt}, J=7.6,3.6 \mathrm{~Hz}), 2.67-2.61(1 \mathrm{H}, \mathrm{m}), 2.36(1 \mathrm{H}, \mathrm{dt}, J$ $=14.0,8.0 \mathrm{~Hz}), 2.22(1 \mathrm{H}, \mathrm{d}, J=3.6 \mathrm{~Hz}) .{ }^{13} \mathrm{C} \mathrm{NMR}\left(\mathrm{CDCl}_{3}, 100 \mathrm{MHz}\right): \delta 142.7,134.3,132.7$, 128.9, 127.7, 127.4, 121.9, 118.8, 72.0, 42.4. Anal Calcd for $\mathrm{C}_{10} \mathrm{H}_{11} \mathrm{BrO}$ : C, 52.89; H, 4.88. Found C, 52.73; H, 4.86. Optical Rotation: $[\alpha]^{25}{ }_{\mathrm{D}}+77^{\circ}\left(\mathrm{c} 1.0, \mathrm{CHCl}_{3}\right){ }^{7}$

Optical purity is established by chiral GLC analysis (Supelco Beta Dex 120 (30 m x 0.15 $\mathrm{mm} \times 0.25 \mu \mathrm{m}$ film thickness), $130{ }^{\circ} \mathrm{C}$ for $20 \mathrm{~min}, 0.5{ }^{\circ} \mathrm{C} /$ minute to $150{ }^{\circ} \mathrm{C}, 15 \mathrm{psi}$ ); chromatograms are illustrated below for an $82 \%$ ee sample:

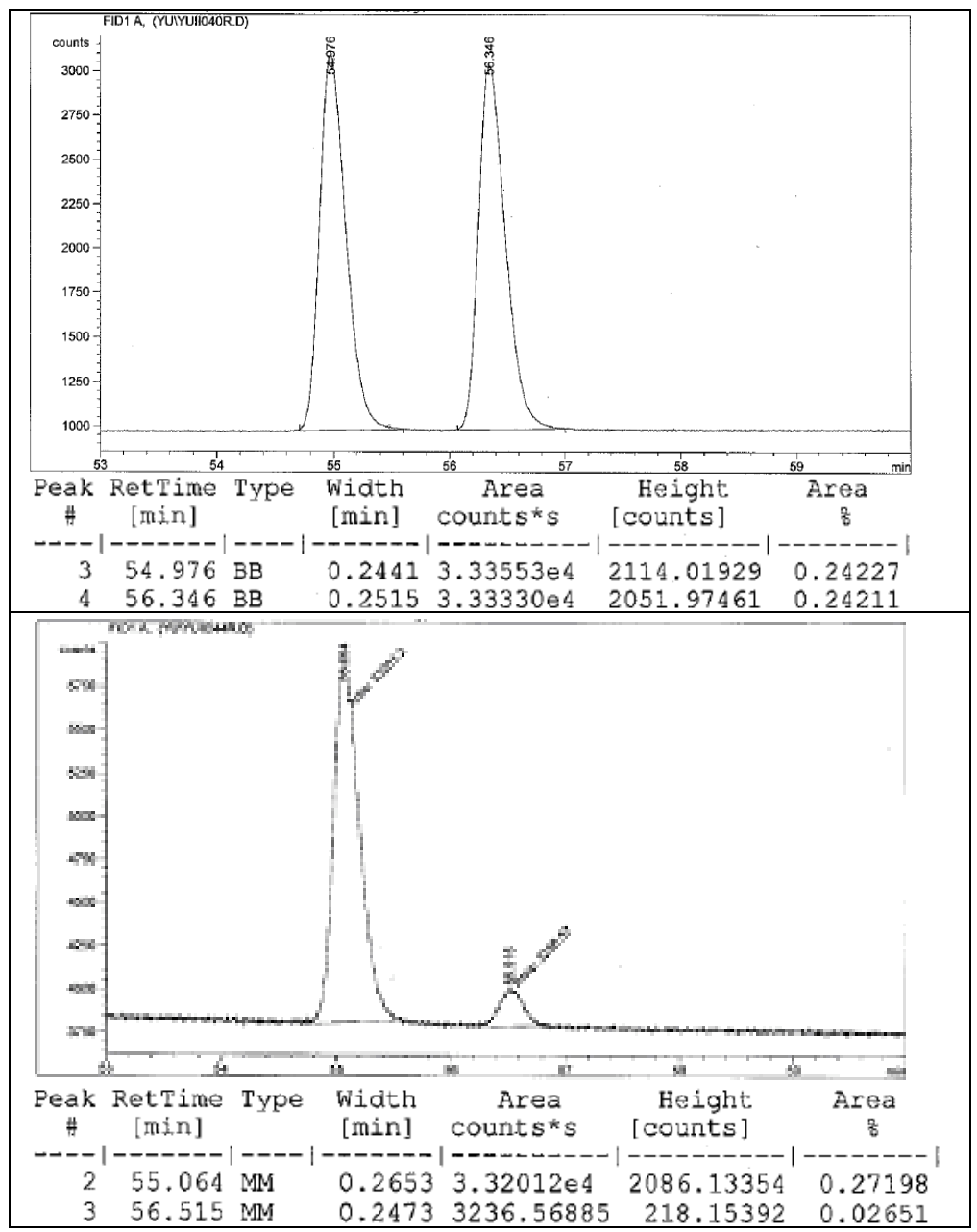

${ }^{7}$ Assigned $(R)$ by analogy. 
<smiles>C=CC[C@H](O)c1ccc(Cl)cc1</smiles>

(R)-1-(4-Chlorophenyl)-3-buten-1-ol (Entry 3, Table 4). The general procedure was followed with ligand $3 \mathrm{r}(32 \mathrm{mg}, 0.10 \mathrm{mmol})$, and $p$-chlorobenzaldehyde (141 mg, $1.00 \mathrm{mmol})$ in DCE $(2.0 \mathrm{~mL}, 0.5 \mathrm{M}$ in substrate). Allyltrichlorosilane $(217 \mu \mathrm{L}, 1.50 \mathrm{mmol})$ was then added. The reaction was sealed and allowed to react for $24 \mathrm{~h}$. After workup, the product was purified by silica gel chromatography (85:15 hexanes:diethyl ether) to yield a pale yellow oil (155 $\mathrm{mg}, 85 \%$ yield, $84 \%$ ee). ${ }^{1} \mathrm{H}$ NMR $\left(\mathrm{CDCl}_{3}, 400 \mathrm{MHz}\right): \delta$ 7.34-7.28 $(4 \mathrm{H}, \mathrm{m}), 5.79(1 \mathrm{H}$, dddd, $J=17.6,9.6,8.0,6.4 \mathrm{~Hz})$, $5.17(1 \mathrm{H}, \mathrm{d}, J=17.6 \mathrm{~Hz}), 5.16(1 \mathrm{H}, \mathrm{d}, J=10.6 \mathrm{~Hz}), 4.73(1 \mathrm{H}, \mathrm{ddd}, J=8.0,4.8,3.2 \mathrm{~Hz}), 2.55-2.41$ $(2 \mathrm{H}, \mathrm{m}), 2.03(1 \mathrm{H}, \mathrm{d}, J=2.8 \mathrm{~Hz}) .{ }^{13} \mathrm{C} \mathrm{NMR}\left(\mathrm{CDCl}_{3}, 100 \mathrm{MHz}\right): \delta 142.3,134.0,133.2,128.6$, 127.3, 119.0, 72.8, 44.2. Optical Rotation: $[\alpha]_{\mathrm{D}}^{25}+62^{\circ}\left(\mathrm{c} 1.0, \mathrm{CHCl}_{3}\right){ }^{8}$

Optical purity is established by chiral GLC analysis (Supelco Beta Dex 120 (30 m x 0.15 $\mathrm{mm} \times 0.25 \mu \mathrm{m}$ film thickness), $100{ }^{\circ} \mathrm{C}$ for $10 \mathrm{~min}, 1{ }^{\circ} \mathrm{C} /$ minute to $180{ }^{\circ} \mathrm{C}, 15 \mathrm{psi}$ ); chromatograms are illustrated below for an $85 \%$ ee sample:

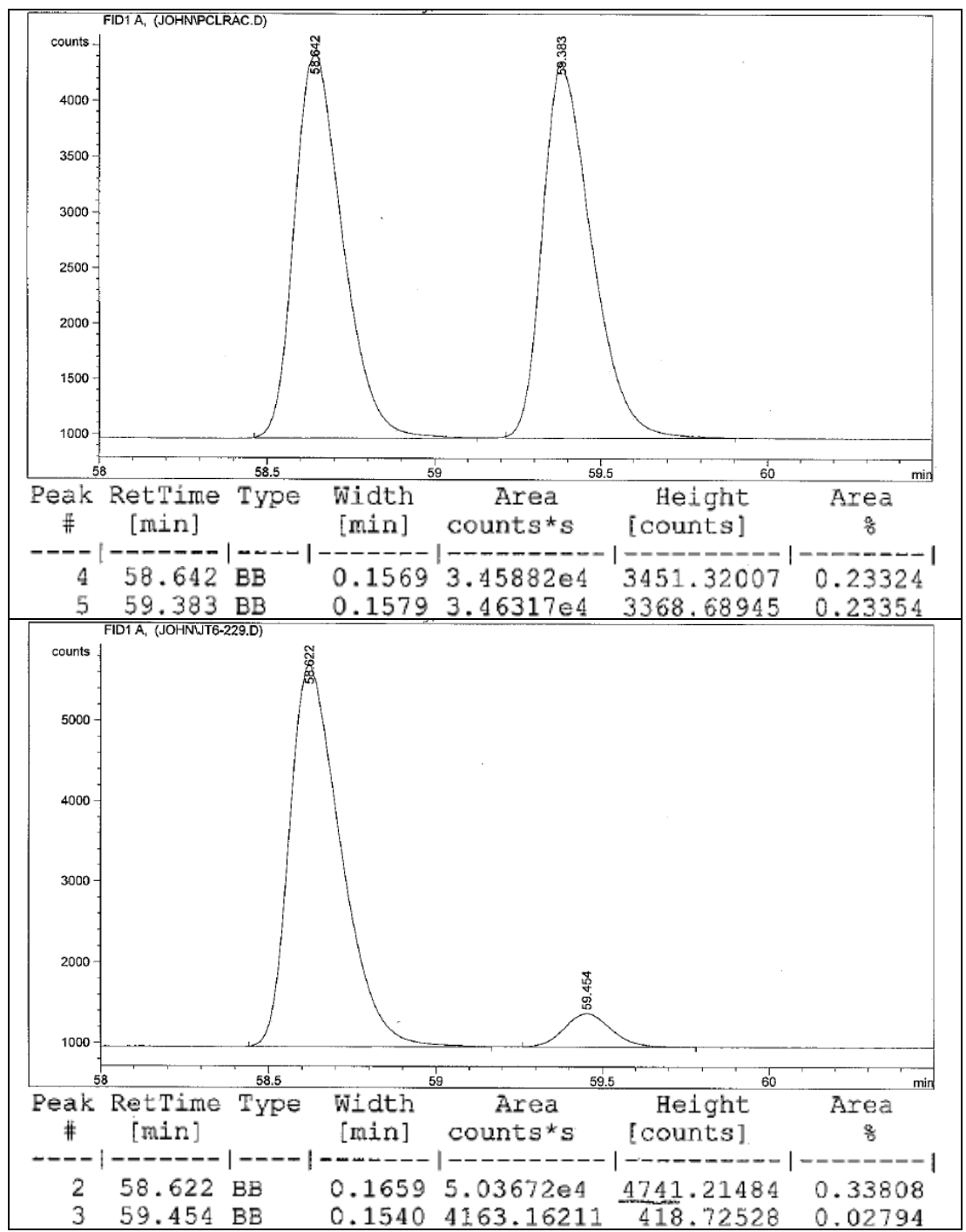

\footnotetext{
${ }^{8}$ Corresponds to $(R)$ enantiomer. ${ }^{1} \mathrm{H}$ NMR data is consistent with published spectrum. See Ref. 6 a.${ }^{13} \mathrm{C}$ NMR is consistent with product.
} 


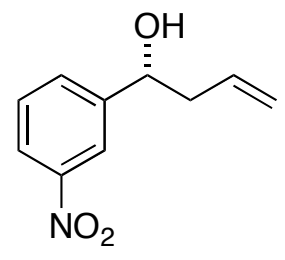

(R)-1-(3-Nitrophenyl)-3-buten-1-ol (Entry 4, Table 4). The general procedure was followed with ligand $3 \mathbf{r}(32 \mathrm{mg}, 0.10 \mathrm{mmol})$, and $m$-nitrobenzaldehyde (151 $\mathrm{mg}, 1.00 \mathrm{mmol})$ in DCE $(2.0 \mathrm{~mL}, 0.5 \mathrm{M}$ in substrate). Allyltrichlorosilane (181 $\mu \mathrm{L}, 1.30 \mathrm{mmol}$ ) was then added. The reaction was sealed and allowed to react for $24 \mathrm{~h}$. After workup, the product was purified by silica gel chromatography (75:25 hexanes:diethyl ether) to yield a yellow oil (146 mg, 76\% yield, $72 \%$ ee). IR (neat, thin film): 3427 (br), 3081 (w), 2911 (w), 1646 (w), 1533 (s), 1350 (s), 1199 (w), 1054 (m), $992(\mathrm{w}), 922(\mathrm{w}), 809(\mathrm{~m}), 740(\mathrm{~m}), 689(\mathrm{~m}) \mathrm{cm}^{-1} .{ }^{1} \mathrm{H} \mathrm{NMR}\left(\mathrm{CDCl}_{3}, 400 \mathrm{MHz}\right): \delta 8.24(1 \mathrm{H}, \mathrm{s}), 8.13$ $(1 \mathrm{H}, \mathrm{d}, J=8.0 \mathrm{~Hz}), 7.70(1 \mathrm{H}, \mathrm{d}, J=7.6 \mathrm{~Hz}), 7.52(1 \mathrm{H}, \mathrm{t}, J=7.6 \mathrm{~Hz}), 5.79(1 \mathrm{H}, \mathrm{dddd}, J=17.2$, $10.8,8.0,6.8 \mathrm{~Hz}), 5.20(1 \mathrm{H}, \mathrm{d}, J=9.6 \mathrm{~Hz}), 5.19(1 \mathrm{H}, \mathrm{d}, J=17.6 \mathrm{~Hz}), 4.88-4.84(1 \mathrm{H}, \mathrm{m}), 2.61-2.43$ $(2 \mathrm{H}, \mathrm{m}), 2.27(1 \mathrm{H}, \mathrm{br}) .{ }^{13} \mathrm{C} \mathrm{NMR}\left(\mathrm{CDCl}_{3}, 100 \mathrm{MHz}\right): \delta 148.4,146.0,133.3,132.0,129.4,122.6$, 121.0, 119.8, 72.3, 44.2. Anal Calcd for $\mathrm{C}_{10} \mathrm{H}_{11} \mathrm{NO}_{3}$ : C, 62.17; H, 5.74; N, 7.25. Found C, 62.00; H, 5.79; N, 7.19. Optical Rotation: $[\alpha]^{25}{ }_{\mathrm{D}}+47^{\circ}\left(\mathrm{c} 1.0, \mathrm{CHCl}_{3}\right){ }^{9}$

Optical purity is established by chiral HPLC analysis (Chiralcel OD $(4.6 \mathrm{~mm} \times 250 \mathrm{~mm})$, 99.5:0.5 hexanes:isopropanol, $0.50 \mathrm{~mL} / \mathrm{min}, \lambda=210 \mathrm{~nm}$.); chromatograms are illustrated below for a $72 \%$ ee sample:

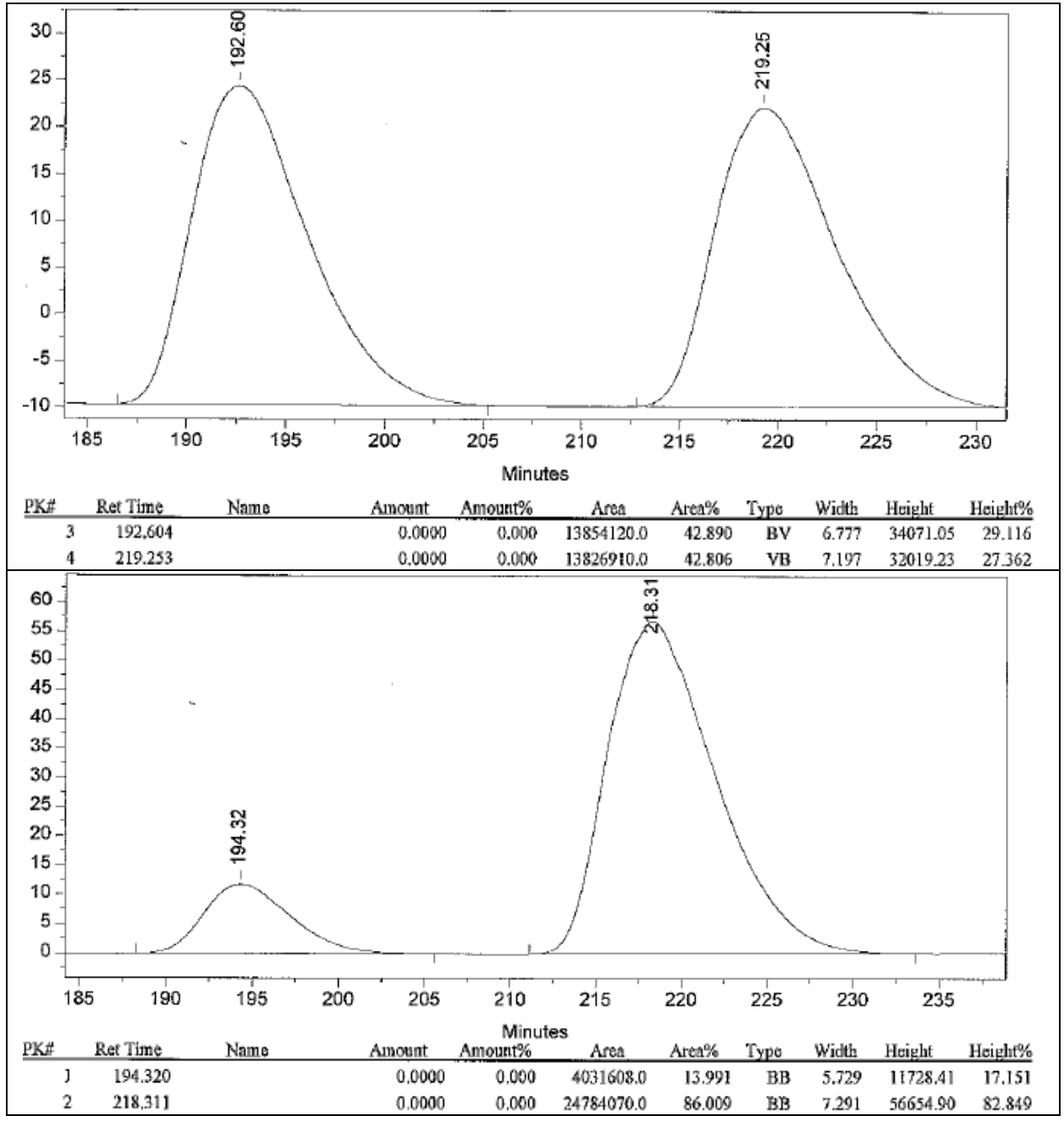

${ }^{9}$ Assigned (R) by analogy. 
<smiles>C=CC[C@H](O)c1ccc(OC)cc1</smiles>

(R)-1-(4-Methoxyphenyl)-3-buten-1-ol (Entry 5, Table 4). The general procedure was followed with ligand $3 \mathbf{r}(32 \mathrm{mg}, 0.10 \mathrm{mmol})$, and $p$ anisaldehyde $(122 \mu \mathrm{L}, 1.00 \mathrm{mmol})$ in DCE $(2.0 \mathrm{~mL}, 0.5 \mathrm{M}$ in substrate). Allyltrichlorosilane $(217 \mu \mathrm{L}, 1.50 \mathrm{mmol})$ was then added. The reaction was sealed and allowed to react for $24 \mathrm{~h}$. After workup, the product was purified by silica gel chromatography (85:15 hexanes:diethyl ether) to yield a pale yellow oil (154 $\mathrm{mg}, 85 \%$ yield, $87 \%$ ee). ${ }^{1} \mathrm{H} \mathrm{NMR}\left(\mathrm{CDCl}_{3}, 400 \mathrm{MHz}\right): \delta 7.28(2 \mathrm{H}, \mathrm{dt}, J=8.4,2.4 \mathrm{~Hz}), 6.89(2 \mathrm{H}, \mathrm{dt}, J=8.0,2.0 \mathrm{~Hz})$, $5.78(1 \mathrm{H}, \mathrm{ddt}, J=17.6,10.4,7.2 \mathrm{~Hz}), 5.16(1 \mathrm{H}, \mathrm{dd}, J=17.6,1.6 \mathrm{~Hz}), 5.13(1 \mathrm{H}, \mathrm{dd}, J=10.4,1.6$ $\mathrm{Hz}), 4.69(1 \mathrm{H}, \mathrm{dt}, J=6.4,2.0 \mathrm{~Hz}), 3.81(3 \mathrm{H}, \mathrm{s}), 2.50(2 \mathrm{H}, \mathrm{t}, J=6.4 \mathrm{~Hz}), 1.98(1 \mathrm{H}, \mathrm{br}) .{ }^{13} \mathrm{C}$ NMR $\left(\mathrm{CDCl}_{3}, 100 \mathrm{MHz}\right): \delta 159.1,136.1,134.7,127.2,118.3,113.9,73.2,55.5,44.1$. Optical Rotation: $[\alpha]^{25}+57^{\circ}\left(\mathrm{c} 1.0, \mathrm{CHCl}_{3}\right){ }^{10}$

Optical purity is established by chiral HPLC analysis (Chiralcel OD (4.6 mm x $250 \mathrm{~mm}$ ), 98:2 hexanes:isopropanol, $1.0 \mathrm{~mL} / \mathrm{min}, \lambda=220 \mathrm{~nm}$.); chromatograms are illustrated below for an $88 \%$ ee sample:

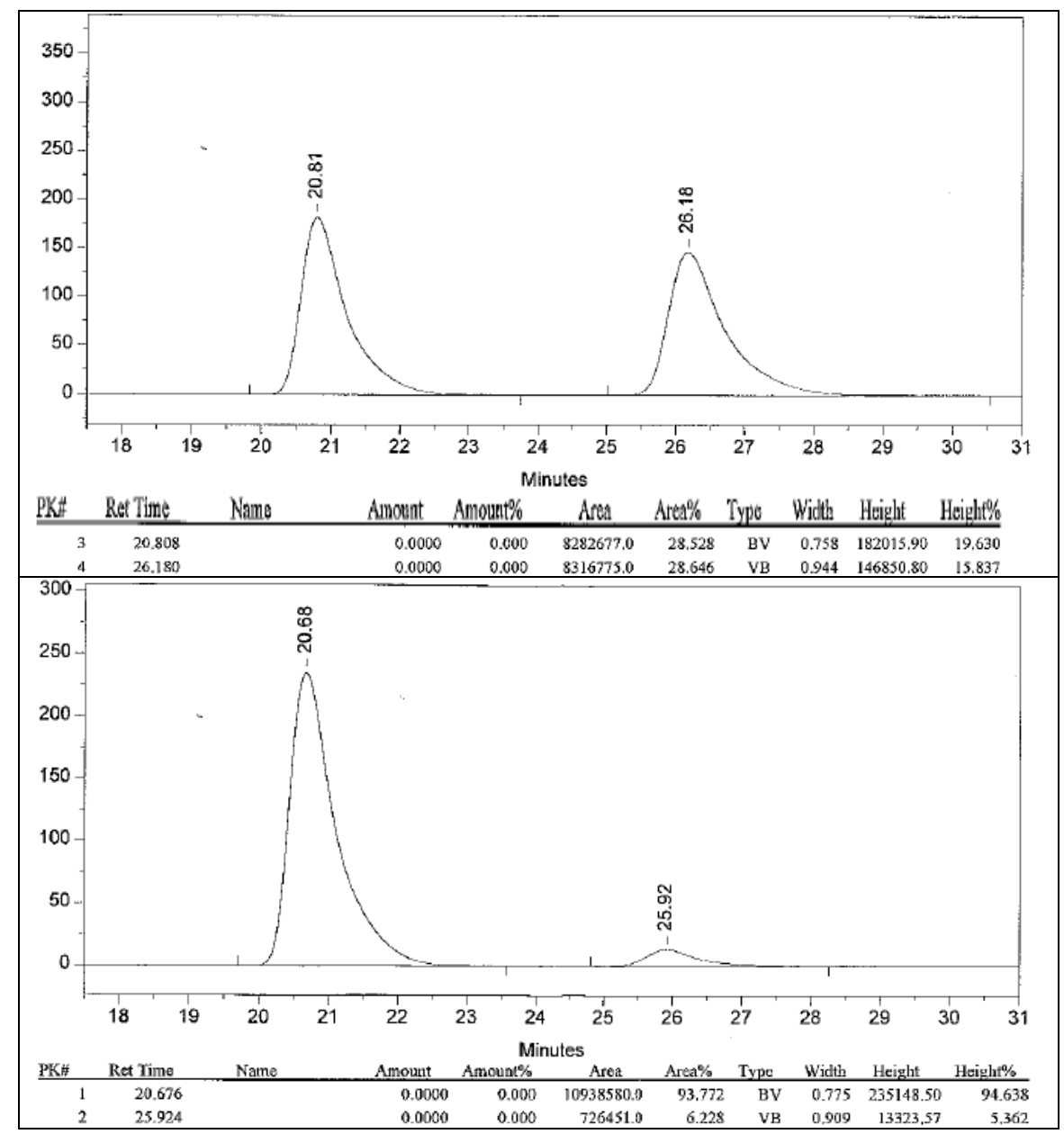

${ }^{10}$ Corresponds to $(R)$ enantiomer. See Ref. $6{ }^{\mathrm{a}} .{ }^{1} \mathrm{H}$ and ${ }^{13} \mathrm{C}$ NMR data are consistent with known product. See Ref. 6 . 
<smiles>C=CC[C@H](O)c1ccc(OC)c(OC)c1</smiles>

(R)-1-(3,4-Dimethoxyphenyl)-3-buten-1-ol (Entry 6, Table 4). The general procedure was followed with ligand $3 \mathbf{r}(47 \mathrm{mg}, 0.15 \mathrm{mmol})$, and 3,4-dimethoxybenzaldehyde (166 mg, $1.00 \mathrm{mmol})$ in DCE $(2.0 \mathrm{~mL}, 0.5 \mathrm{M}$ in substrate). Allyltrichlorosilane $(181 \mu \mathrm{L}, 1.30 \mathrm{mmol})$ was then added.

The reaction was sealed and allowed to react for $24 \mathrm{~h}$. After workup, the product was purified by silica gel chromatography (70:30 hexanes:diethyl ether) to yield a white solid $(151 \mathrm{mg}, 73 \%$ yield, 92\% ee). M.P. $94-95{ }^{\circ} \mathrm{C} .{ }^{1} \mathrm{H} \mathrm{NMR}\left(\mathrm{CDCl}_{3}, 400 \mathrm{MHz}\right): \delta 6.93(1 \mathrm{H}, \mathrm{d}, J=2.0 \mathrm{~Hz}), 6.88(1 \mathrm{H}, \mathrm{dd}, J=$ $8.4,2.0 \mathrm{~Hz}), 6.83(1 \mathrm{H}, \mathrm{d}, J=8.4 \mathrm{~Hz}), 5.81(1 \mathrm{H}, \mathrm{ddt}, J=17.7,10.4,7.6 \mathrm{~Hz}), 5.17(1 \mathrm{H}, \mathrm{dd}, J=17.2$, $2.0 \mathrm{~Hz}), 5.14(1 \mathrm{H}, \mathrm{dd}, J=9.6,2.0 \mathrm{~Hz}), 4.68(1 \mathrm{H}, \mathrm{t}, J=6.8 \mathrm{~Hz}), 3.89(3 \mathrm{H}, \mathrm{s}), 3.87(3 \mathrm{H}, \mathrm{s}), 2.50(2 \mathrm{H}$, $\mathrm{t}, J=7.2 \mathrm{~Hz}), 2.03(1 \mathrm{H}, \mathrm{br}) .{ }^{13} \mathrm{C} \mathrm{NMR}\left(\mathrm{CDCl}_{3}, 100 \mathrm{MHz}\right): \delta 149.2,148.6,136.8,134.7,118.5$, 118.3, 111.2, 109.3, 73.5, 56.3, 56.2, 44.2. Optical Rotation: $[\alpha]^{25}{ }_{\mathrm{D}}+30^{\circ}\left(\mathrm{c} 1.0, \mathrm{C}_{6} \mathrm{H}_{6}\right) .^{11}$

Optical purity is established by chiral HPLC analysis (Chiralcel AS (4.6 mm x $250 \mathrm{~mm}$ ), 99.5:0.5 hexanes:isopropanol, $0.75 \mathrm{~mL} / \mathrm{min}, \lambda=220 \mathrm{~nm}$.); chromatograms are illustrated below for a $92 \%$ ee sample:

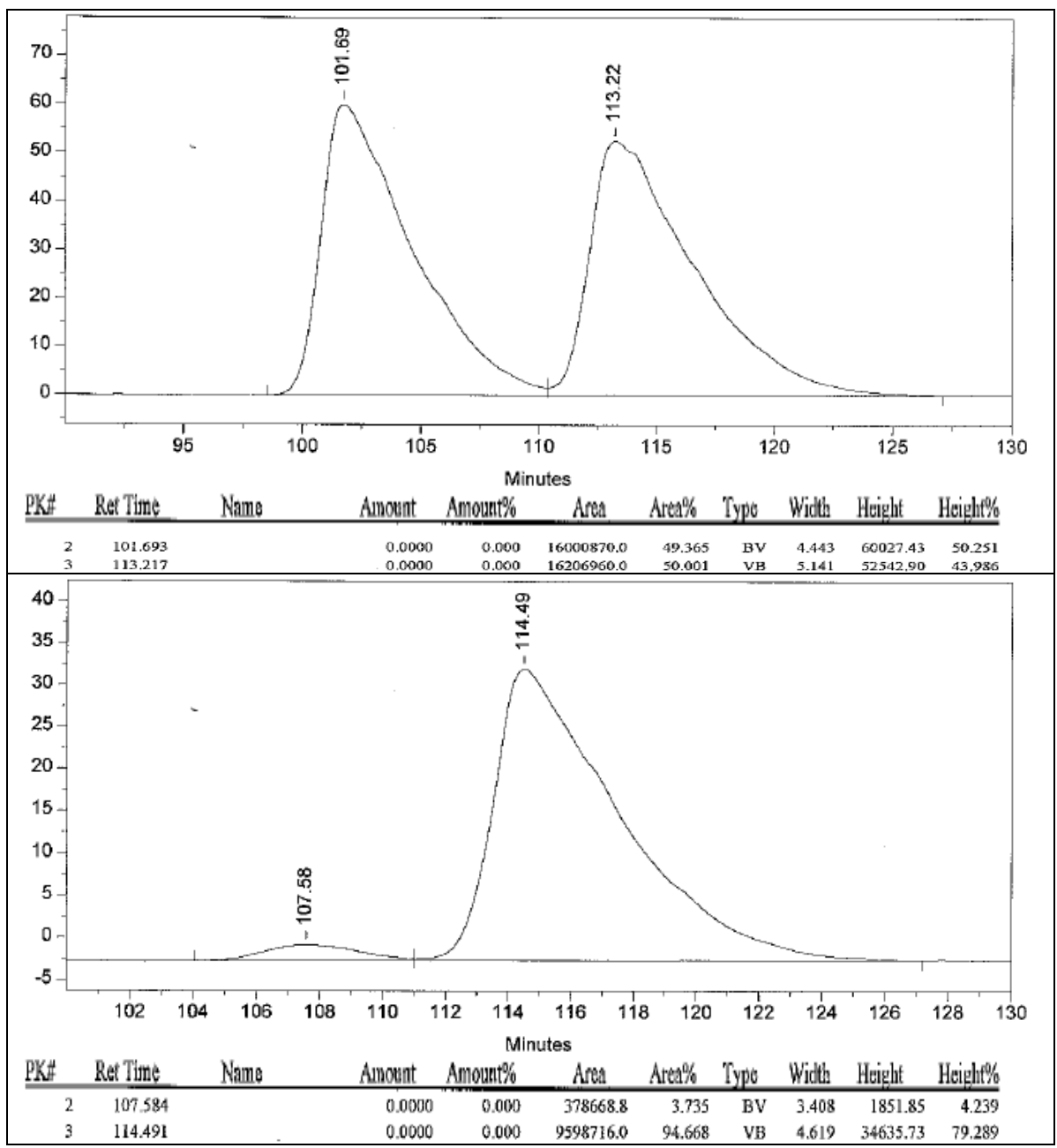

${ }^{11}$ Corresponds to $(R)$ enantiomer. ${ }^{1} \mathrm{H}$ and ${ }^{13} \mathrm{C}$ NMR data are consistent with published. See Shimada, T.; Kina, A.; Hayashi, T. J. Org. Chem. 2003, 68, 6329-6337. 
<smiles>C=CC[C@H](O)c1cccc2ccccc12</smiles>

(R)-1-(1-Naphthyl)-3-buten-1-ol (Entry 7, Table 4). The general procedure was followed with ligand $3 \mathbf{r}(32 \mathrm{mg}, 0.10 \mathrm{mmol})$, and 1-naphthaldehyde (156 $\mathrm{mg}, 1.00 \mathrm{mmol})$ in DCE (2.0 mL, 0.5 M in substrate). Allyltrichlorosilane (181 $\mu \mathrm{L}, 1.30 \mathrm{mmol})$ was then added. The reaction was sealed and allowed to react for $24 \mathrm{~h}$. After workup, the product was purified by silica gel chromatography (95:5 hexanes:diethyl ether) to yield a yellow oil $\left(176 \mathrm{mg}, 89 \%\right.$ yield, $79 \%$ ee). ${ }^{1} \mathrm{H} \mathrm{NMR}\left(\mathrm{CDCl}_{3}, 400 \mathrm{MHz}\right): \delta 8.09(1 \mathrm{H}$, $\mathrm{d}, J=8.0 \mathrm{~Hz}), 7.89(1 \mathrm{H}, \mathrm{d}, J=8.4 \mathrm{~Hz}), 7.79(1 \mathrm{H}, \mathrm{d}, J=8.0 \mathrm{~Hz}), 7.68(1 \mathrm{H}, \mathrm{d}, J=6.8 \mathrm{~Hz}), 7.55-7.47$ $(3 \mathrm{H}, \mathrm{m}), 5.94(1 \mathrm{H}, \mathrm{ddt}, J=17.2,10.0,6.8 \mathrm{~Hz}), 5.55(1 \mathrm{H}, \mathrm{dd}, J=8.4,4.0 \mathrm{~Hz}), 5.23(1 \mathrm{H}, \mathrm{d}, J=17.2$ $\mathrm{Hz}), 5.20(1 \mathrm{H}, \mathrm{d}, J=10.8 \mathrm{~Hz}), 2.81-2.75(1 \mathrm{H}, \mathrm{m}), 2.66-2.58(1 \mathrm{H}, \mathrm{m}) .{ }^{13} \mathrm{C} \mathrm{NMR}\left(\mathrm{CDCl}_{3}, 100 \mathrm{MHz}\right)$ : $\delta 139.5,134.8,133.9,130.4,129.0,128.1,126.1,125.6,125.5,123.0,122.9,118.5,70.2,43.2$. Optical Rotation: $[\alpha]^{25}+84^{\circ}\left(\mathrm{c} 1.0, \mathrm{CHCl}_{3}\right){ }^{12}$

Optical purity is established by chiral HPLC analysis (Chiralcel OD (4.6 mm x $250 \mathrm{~mm}$ ), 90:10 hexanes:isopropanol, $1.0 \mathrm{~mL} / \mathrm{min}, \lambda=254 \mathrm{~nm}$.); chromatograms are illustrated below for a $79 \%$ ee sample:

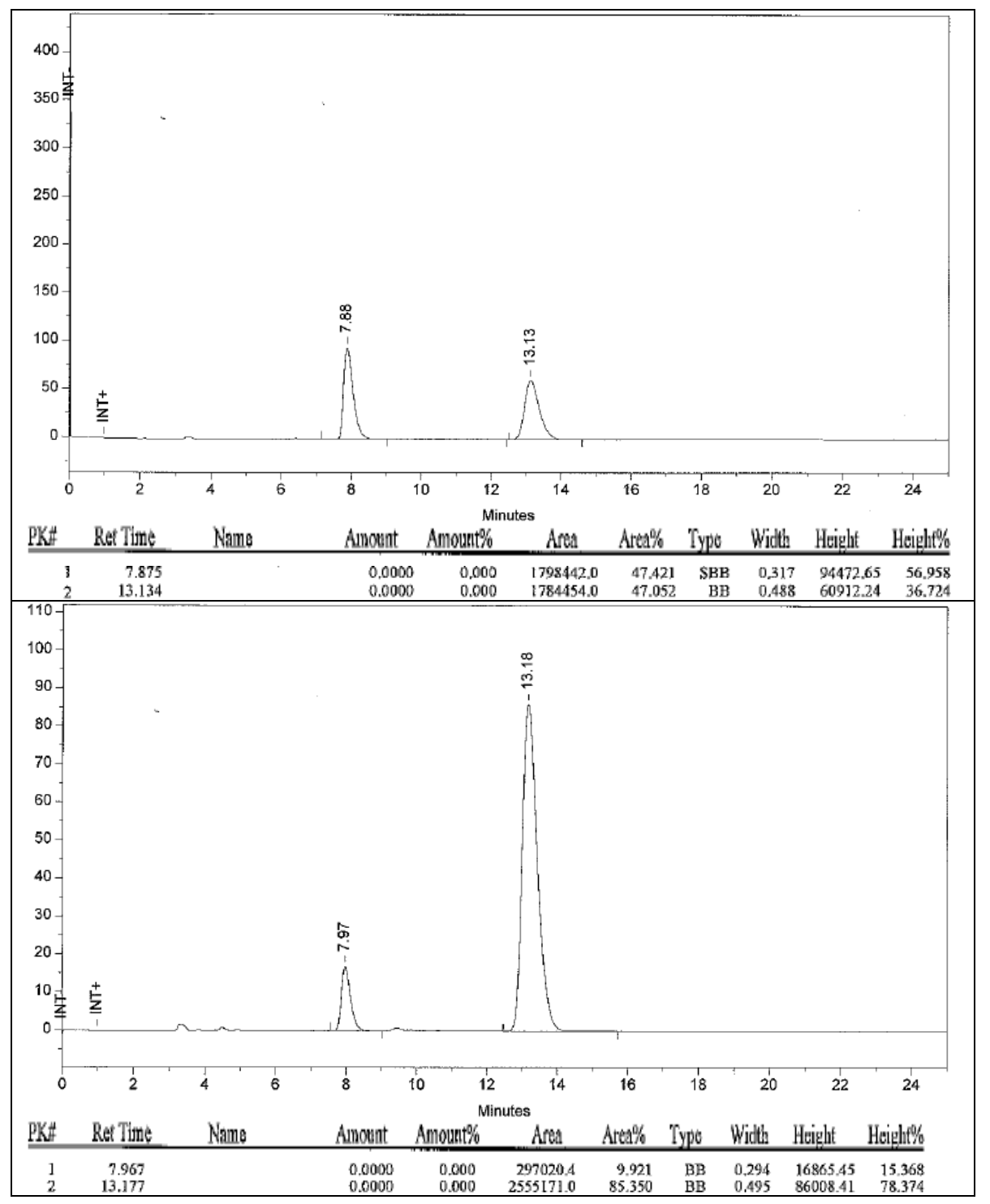

${ }^{12}$ Corresponds to $(R)$ enantiomer. See Ref. $6{ }^{\mathrm{a}} .{ }^{1} \mathrm{H}$ and ${ }^{13} \mathrm{C}$ NMR data are consistent with known product. 
<smiles>C=CC[C@H](O)c1ccc2ccccc2c1</smiles>

(R)-1-(2-Naphthyl)-3-buten-1-ol (Entry 8, Table 4). The general procedure was followed with ligand $3 \mathbf{r}(32 \mathrm{mg}, 0.10 \mathrm{mmol})$, and 2-naphthaldehyde (156 mg, $1.00 \mathrm{mmol})$ in DCE $(2.0 \mathrm{~mL}, 0.5 \mathrm{M}$ in substrate). Allyltrichlorosilane $(181 \mu \mathrm{L}, 1.30 \mathrm{mmol})$ was then added. The reaction was sealed and allowed to react for $24 \mathrm{~h}$. After workup, the product was purified by silica gel chromatography (95:5 hexanes:diethyl ether) to yield a white solid (177 mg, 89\% yield, 83\% ee). M.P. $34-35{ }^{\circ} \mathrm{C} .{ }^{1} \mathrm{H}$ NMR $\left(\mathrm{CDCl}_{3}, 400 \mathrm{MHz}\right): \delta$ 7.86-7.82 (4H, m), 7.50-7.45 (3H, m), 5.84 (1H, ddt, $J=17.2,10.4,7.2 \mathrm{~Hz}), 5.20(1 \mathrm{H}, \mathrm{d}, J=18.0 \mathrm{~Hz}), 5.16(1 \mathrm{H}, \mathrm{d}, J=10.4 \mathrm{~Hz}), 4.95(1 \mathrm{H}, \mathrm{dd}, J=7.2$, $4.8 \mathrm{~Hz}), 2.68-2.55(2 \mathrm{H}, \mathrm{m}), 2.11(1 \mathrm{H}, \mathrm{br}) .{ }^{13} \mathrm{C} \mathrm{NMR}\left(\mathrm{CDCl}_{3}, 100 \mathrm{MHz}\right): \delta 141.3,134.4,133.4$, $133.1,128.3,128.1,127.8,126.2,126.0,124.6,124.1,118.7,73.6,44.1$. Optical Rotation: $[\alpha]^{25}$ $+58^{\circ}\left(\mathrm{c} 1.0, \mathrm{CHCl}_{3}\right){ }^{13}$

Optical purity is established by chiral HPLC analysis (Chiralcel AS (4.6 mm x $250 \mathrm{~mm}$ ), 99:1 hexanes:isopropanol, $0.50 \mathrm{~mL} / \mathrm{min}, \lambda=254 \mathrm{~nm}$.); chromatograms are illustrated below for an $83 \%$ ee sample:

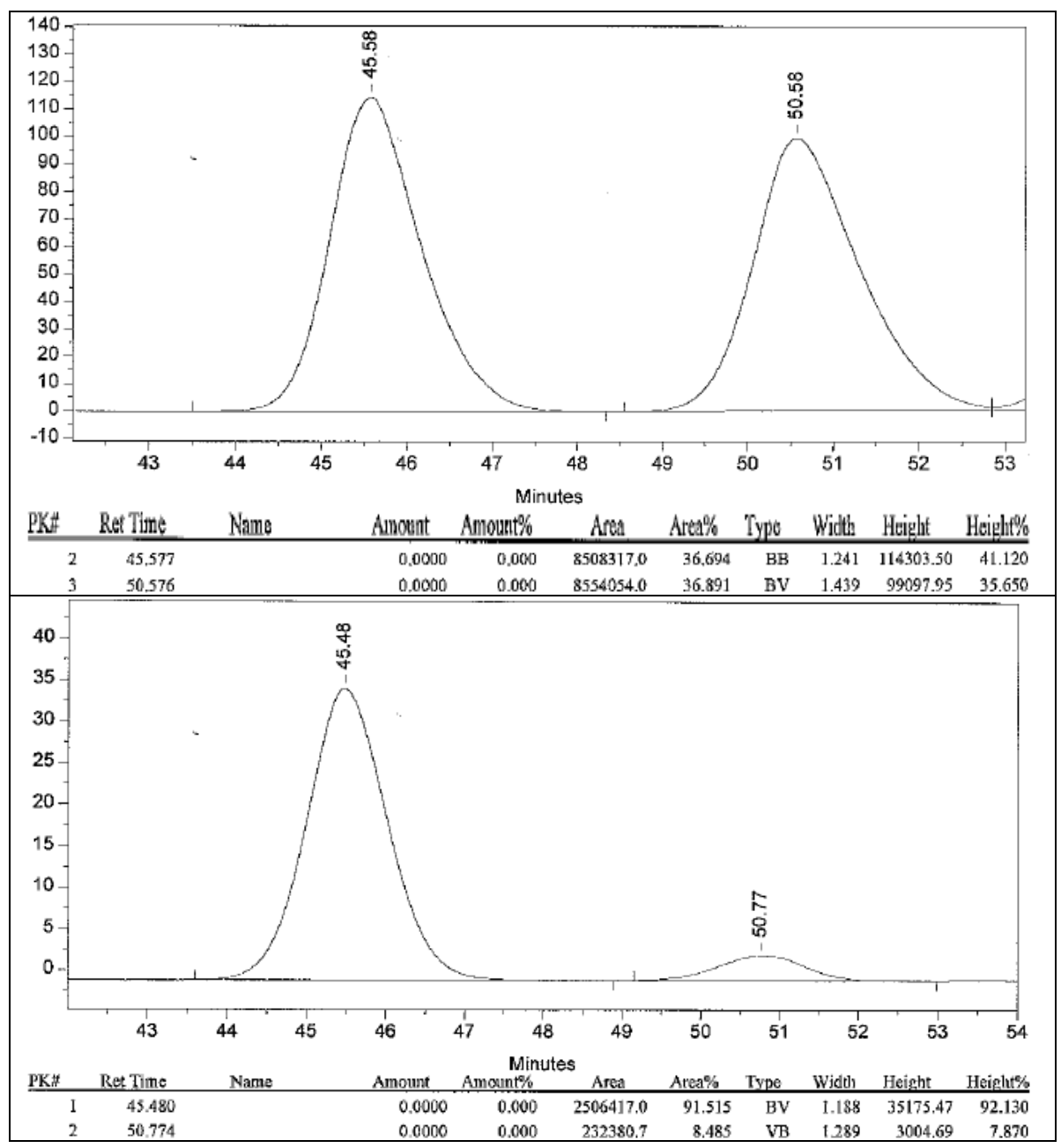

${ }^{13}$ Corresponds to $(R)$ enantiomer. See Ref. $6{ }^{\mathrm{a}} \cdot{ }^{1} \mathrm{H}$ and ${ }^{13} \mathrm{C}$ NMR data are consistent with known product. See Ref. 6 . 
<smiles>C=CC[C@H](O)c1ccco1</smiles>

(R)-1-(2-Furyl)-3-buten-1-ol (Entry 9, Table 4). The general procedure was followed with ligand $3 \mathbf{r}(32 \mathrm{mg}, 0.10 \mathrm{mmol})$, and 2-furaldehyde $(83 \mu \mathrm{L}, 1.0$ $\mathrm{mmol})$ in DCE $(2.0 \mathrm{~mL}, 0.5 \mathrm{M}$ in substrate). Allyltrichlorosilane $(181 \mu \mathrm{L}, 1.30$ mmol) was then added. The reaction was sealed and allowed to react for $24 \mathrm{~h}$. After workup, the product was purified by silica gel chromatography (90:10 hexanes:diethyl ether) to yield a yellow oil $\left(81 \mathrm{mg}, 59 \%\right.$ yield, $71 \%$ ee). ${ }^{1} \mathrm{H}$ NMR $\left(\mathrm{CDCl}_{3}, 400 \mathrm{MHz}\right): \delta 7.38(1 \mathrm{H}, \mathrm{dd}, J=2.0,0.8 \mathrm{~Hz}), 6.33$ $(1 \mathrm{H}, \mathrm{dd}, J=3.6,2.0 \mathrm{~Hz}), 6.23(1 \mathrm{H}, \mathrm{dd}, J=3.6,2.0 \mathrm{~Hz}), 5.81(1 \mathrm{H}, \mathrm{ddt}, J=17.2,10.0,6.8 \mathrm{~Hz}), 5.19$ $(1 \mathrm{H}, \mathrm{d}, J=17.2 \mathrm{~Hz}), 5.15(1 \mathrm{H}, \mathrm{d}, J=10.0 \mathrm{~Hz}), 4.75(1 \mathrm{H}, \mathrm{br}), 2.69-2.57(2 \mathrm{H}, \mathrm{m}), 2.05(1 \mathrm{H}, \mathrm{br}) .{ }^{13} \mathrm{C}$ NMR $\left(\mathrm{CDCl}_{3}, 100 \mathrm{MHz}\right): \delta 156.1,142.0,133.8,118.7,110.3,106.2,67.2,40.4$. Optical Rotation: $[\alpha]^{25}+10^{\circ}(\mathrm{c} 1.0, \mathrm{EtOH}){ }^{14}$

Optical purity is established by chiral HPLC analysis (Chiralcel AS (4.6 mm x $250 \mathrm{~mm}$ ), 99.5:0.5 hexanes:isopropanol, $0.50 \mathrm{~mL} / \mathrm{min}, \lambda=220 \mathrm{~nm}$.); chromatograms are illustrated below for a $72 \%$ ee sample:

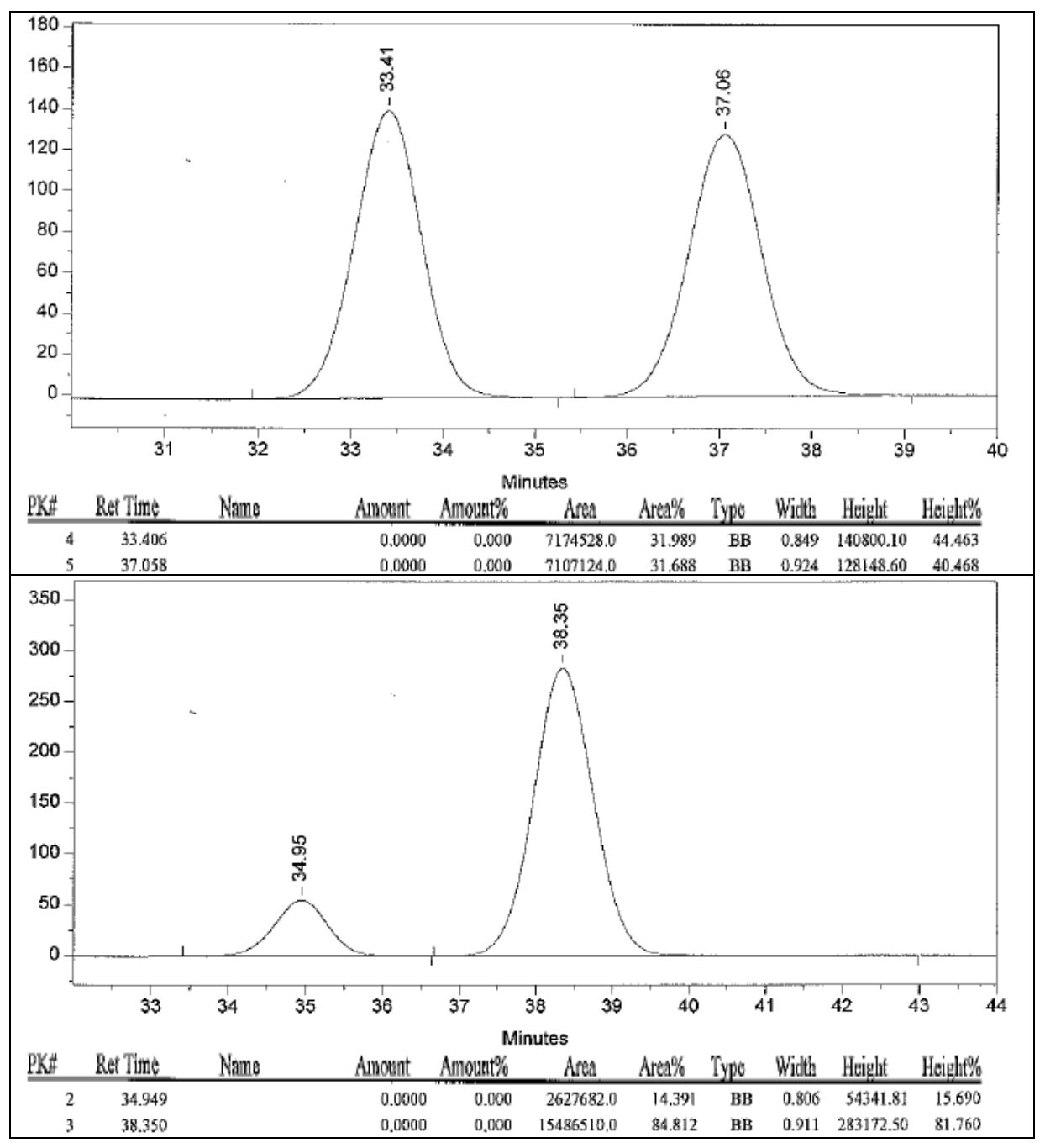

${ }^{14}$ Corresponds to $(R)$ enantiomer. See Ref. $6{ }^{\mathrm{a}} .{ }^{1} \mathrm{H}$ and ${ }^{13} \mathrm{C}$ NMR data are consistent with published. See Racherla, U. S.; Liao, Y.; Brown, H. C. J. Org. Chem. 1992, 57, 6614-6617. 
<smiles>C=CC[C@H](O)c1ccoc1</smiles>

(R)-1-(1-Furyl)-3-buten-1-ol (Entry 10, Table 4). The general procedure was followed with ligand $3 \mathrm{r}(32 \mathrm{mg}, 0.10 \mathrm{mmol})$, and 3 -furaldehyde $(87 \mu \mathrm{L}, 1.0$ mmol) in DCE $(2.0 \mathrm{~mL}, 0.5 \mathrm{M}$ in substrate). Allyltrichlorosilane $(181 \mu \mathrm{L}, 1.30$ mmol) was then added. The reaction was sealed and allowed to react for $24 \mathrm{~h}$. After workup, the product was purified by silica gel chromatography (90:10 hexanes:diethyl ether) to yield a yellow oil (88 mg, 64\% yield, 82\% ee). ${ }^{1} \mathrm{H} \mathrm{NMR}\left(\mathrm{CDCl}_{3}, 400 \mathrm{MHz}\right): \delta 7.39(2 \mathrm{H}$, br), $6.41(1 \mathrm{H}, \mathrm{s}), 5.82(1 \mathrm{H}, \mathrm{ddt}, J=16.8,10.4,6.8 \mathrm{~Hz}), 5.17(1 \mathrm{H}, \mathrm{d}, J=16.8 \mathrm{~Hz}), 5.15(1 \mathrm{H}, \mathrm{d}, J=10.4$ $\mathrm{Hz}), 4.72(1 \mathrm{H}, \mathrm{br}), 2.57-2.45(2 \mathrm{H}, \mathrm{m}), 1.95$ (1H, br). ${ }^{13} \mathrm{C} \mathrm{NMR}\left(\mathrm{CDCl}_{3}, 100 \mathrm{MHz}\right): \delta 143.4,139.1$, 134.2, 128.6, 118.7, 108.7, 66.4, 42.8. Optical Rotation: $[\alpha]^{25}{ }_{\mathrm{D}}+11^{\circ}(\mathrm{c} 1.0, \mathrm{EtOH}) .^{15}$

Optical purity is established by chiral HPLC analysis (Chiralcel AS (4.6 mm x $250 \mathrm{~mm}$ ), 99:1 hexanes:isopropanol, $0.50 \mathrm{~mL} / \mathrm{min}, \lambda=220 \mathrm{~nm}$.); chromatograms are illustrated below for an $82 \%$ ee sample:

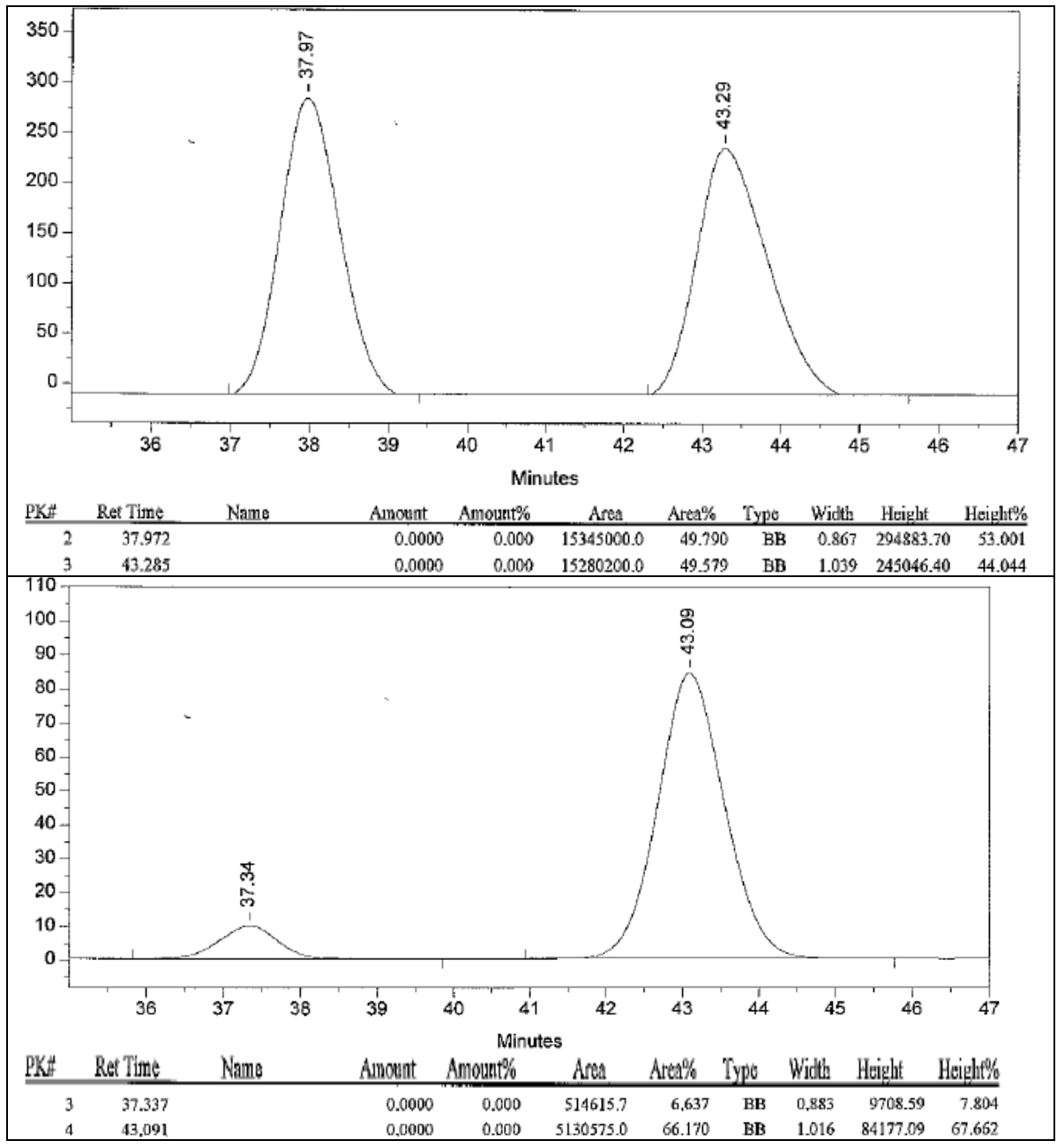

${ }^{15}$ Corresponds to $(R)$ enantiomer. ${ }^{1} \mathrm{H}$ and ${ }^{13} \mathrm{C}$ NMR data are consistent with published data. 


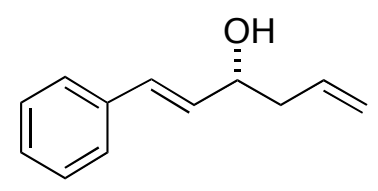

$(1 E, 3 R)$-1-Phenyl-1,5-hexadiene-3-ol $(\mathrm{R}=\mathrm{H}$, eq 1$)$. The general procedure was followed with ligand $\mathbf{3 r}(32 \mathrm{mg}, 0.10 \mathrm{mmol})$, and trans-cinnamaldehyde $(126 \mu \mathrm{L}, 1.0 \mathrm{mmol})$ in DCE $(2.0 \mathrm{~mL}, 0.5 \mathrm{M}$ in substrate). Allyltrichlorosilane $(181 \mu \mathrm{L}, 1.3 \mathrm{mmol})$ was then added. The reaction was sealed and allowed to react for $24 \mathrm{~h}$. After workup, the product was purified by silica gel chromatography (90:10 hexanes:diethyl ether) to yield a yellow oil $(137 \mathrm{mg}, 79 \%$ yield, $71 \%$ ee). ${ }^{1} \mathrm{H}$ NMR $\left(\mathrm{CDCl}_{3}, 400 \mathrm{MHz}\right): \delta 7.38(2 \mathrm{H}, \mathrm{d}, J=7.6 \mathrm{~Hz}), 7.32(1 \mathrm{H}, \mathrm{t}, J=8.0 \mathrm{~Hz}), 7.24(1 \mathrm{H}, \mathrm{t}, J$ $=7.2 \mathrm{~Hz}), 6.61(1 \mathrm{H}, \mathrm{d}, J=16.0 \mathrm{~Hz}), 6.25(1 \mathrm{H}, \mathrm{dd}, J=16.0,6.4 \mathrm{~Hz}), 5.86(1 \mathrm{H}, \mathrm{ddt}, J=17.6,10.0$, $7.2 \mathrm{~Hz}), 5.19(1 \mathrm{H}, \mathrm{d}, J=17.6 \mathrm{~Hz}), 5.17(1 \mathrm{H}, \mathrm{d}, J=10.0 \mathrm{~Hz}), 4.36(1 \mathrm{H}, \mathrm{br}), 2.48-2.35(2 \mathrm{H}, \mathrm{m}), 1.88$ $(1 \mathrm{H}, \mathrm{br}) .{ }^{13} \mathrm{C} \mathrm{NMR}\left(\mathrm{CDCl}_{3}, 100 \mathrm{MHz}\right): \delta 136.7,134.1,131.6,130.4,128.6,127.7,126.6,118.6$, 71.9, 42.3. Optical Rotation: $[\alpha]^{25}+23^{\circ}\left(\mathrm{c} 1.0, \mathrm{CHCl}_{3}\right) .{ }^{16}$

Optical purity is established by chiral HPLC analysis (Chiralcel OD (4.6 $\mathrm{mm} \times 250 \mathrm{~mm})$, 90:10 hexanes:isopropanol, $0.75 \mathrm{~mL} / \mathrm{min}, \lambda=220 \mathrm{~nm}$.); chromatograms are illustrated below for a $71 \%$ ee sample:

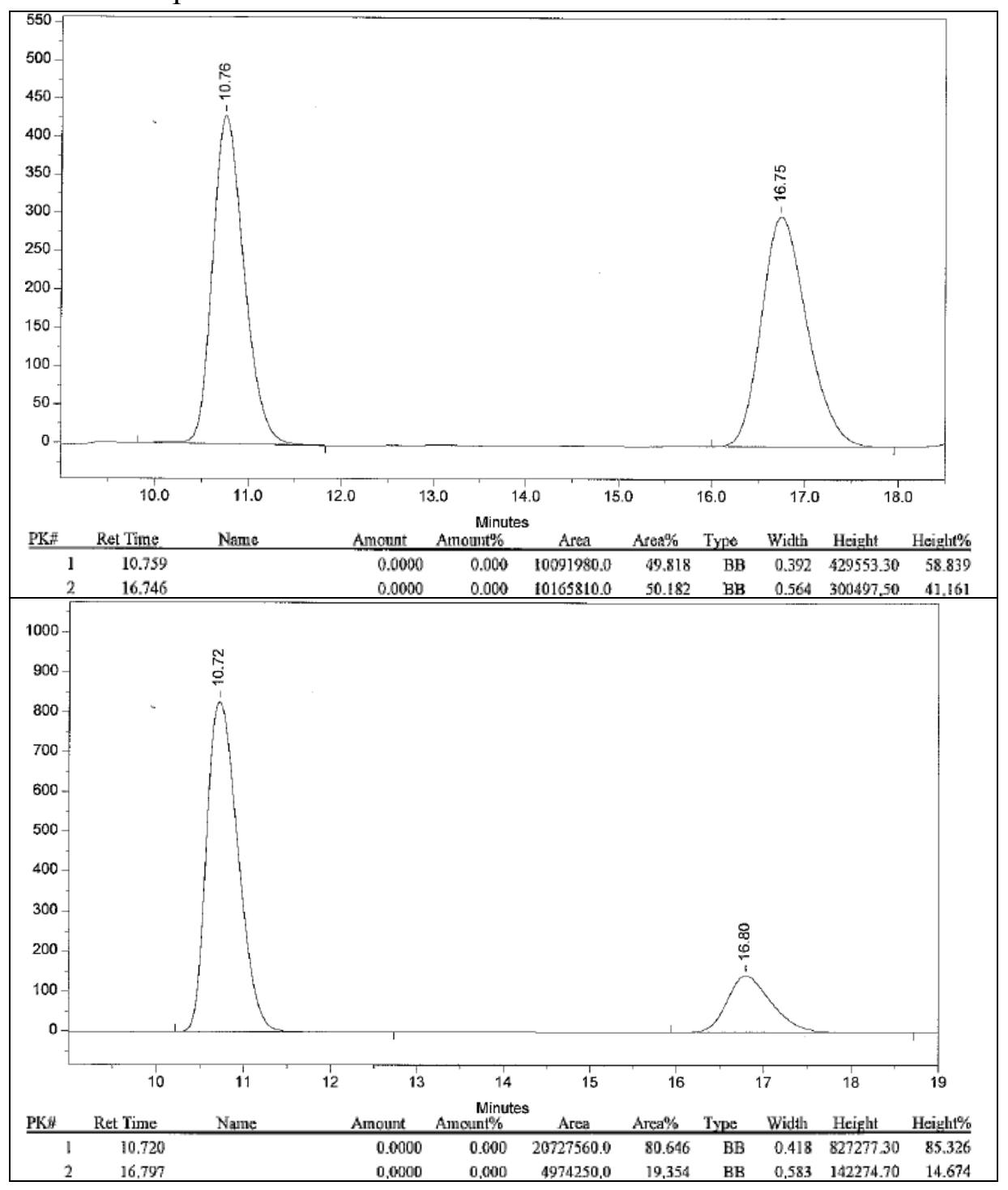

${ }^{16}$ Corresponds to $(R)$ enantiomer, See Ref. $6{ }^{\mathrm{a}} \cdot{ }^{1} \mathrm{H}$ and ${ }^{13} \mathrm{C}$ NMR data are consistent with published. 
<smiles>C=CC[C@@H](O)/C(C)=C/c1ccccc1</smiles>

(1E,3R)-1-Methyl-1-phenyl-1,5-hexadiene-3-ol $(\mathrm{R}=\mathrm{Me}$, eq 1). The general procedure was followed with ligand $3 \mathrm{r}(32 \mathrm{mg}, 0.10 \mathrm{mmol})$, and $\alpha$-methyltrans-cinnamaldehyde $(142 \mu \mathrm{L}, 1.0 \mathrm{mmol})$ in DCE $(2.0 \mathrm{~mL}, 0.5 \mathrm{M}$ in substrate). Allyltrichlorosilane $(181 \mu \mathrm{L}, 1.3 \mathrm{mmol})$ was then added. The reaction was sealed and allowed to react for $24 \mathrm{~h}$. After workup, the product was purified by silica gel chromatography (96:4 hexanes:diethyl ether) to yield a yellow oil (117 mg, 62\% yield, 76\% ee). IR (neat, thin film): 3364 (br), 3075 (w), 3024 (w), 2980 (w), 2917 (w), 1639 (w), 1489 (w), 1445 (m), $998(\mathrm{~m}), 916(\mathrm{~m}), 752(\mathrm{~m}), 712(\mathrm{~s}), 513(\mathrm{w}) \mathrm{cm}^{-1} .{ }^{1} \mathrm{H} \mathrm{NMR}\left(\mathrm{CDCl}_{3}, 400 \mathrm{MHz}\right): \delta$ 7.40-7.30 $(4 \mathrm{H}, \mathrm{m}), 7.27(\mathrm{t}, 1 \mathrm{H}, J=7.2 \mathrm{~Hz}), 6.58(1 \mathrm{H}, \mathrm{s}), 5.89(1 \mathrm{H}, \mathrm{ddt}, J=17.6,10.0,7.2 \mathrm{~Hz}), 5.24(1 \mathrm{H}, \mathrm{d}, J$ $=17.6 \mathrm{~Hz}), 5.20(1 \mathrm{H}, \mathrm{d}, J=10.0 \mathrm{~Hz}), 4.28(1 \mathrm{H}, \mathrm{br}), 2.55-2.41(2 \mathrm{H}, \mathrm{m}), 1.94(3 \mathrm{H}, \mathrm{s}), 1.89(1 \mathrm{H}, \mathrm{d}$, $2.8 \mathrm{~Hz}) .{ }^{13} \mathrm{C} \mathrm{NMR}\left(\mathrm{CDCl}_{3}, 100 \mathrm{MHz}\right): \delta 139.6,137.6,134.6,129.1,128.2,126.5,125.8,118.1$, 76.8, 40.4, 14.0. Anal Calcd for $\mathrm{C}_{13} \mathrm{H}_{16} \mathrm{O}: \mathrm{C}, 82.94 ; \mathrm{H}, 8.57$. Found $\mathrm{C}, 82.64 ; \mathrm{H}, 8.72$. Optical Rotation: $[\alpha]^{25}+2.6^{\circ}\left(\mathrm{c} 1.0, \mathrm{CHCl}_{3}\right){ }^{17}$

Optical purity is established by chiral GLC analysis (Supelco Alpha Dex 120 (30 m x 0.15 $\mathrm{mm} \times 0.25 \mu \mathrm{m}$ film thickness), $120^{\circ} \mathrm{C}$ isothermal, $15 \mathrm{psi}$ ); chromatograms are illustrated below for a $76 \%$ ee sample:

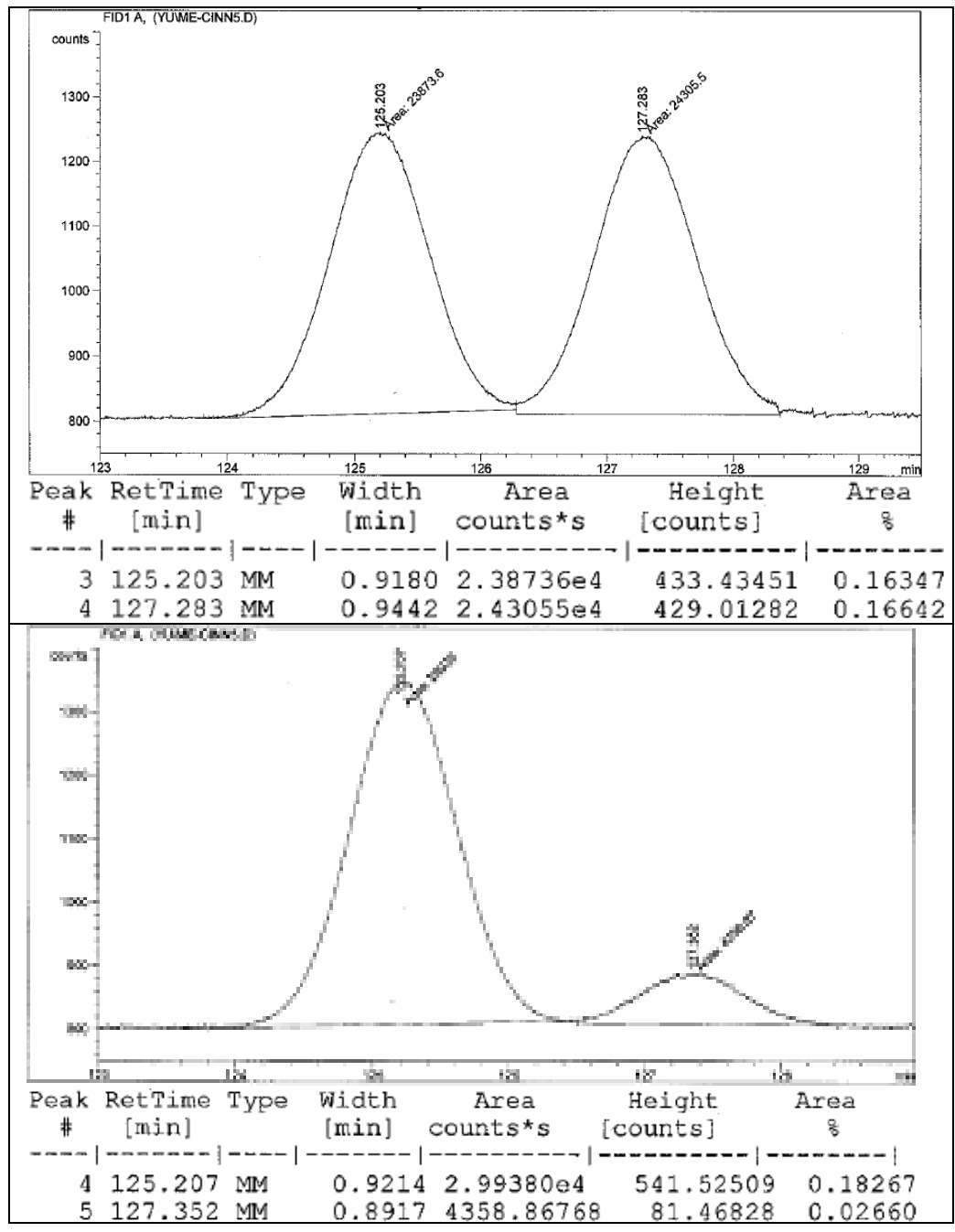

${ }^{17}$ Assigned $(R)$ by analogy. 
$\stackrel{\mathrm{OH}}{(\mathbf{1} R, 2 R)-2-M e t h y l-1-p h e n y l-3-b u t e n-1-0 l(6) .} .^{18}$ The general procedure was followed with ligand $3 \mathbf{r}(32 \mathrm{mg}, 0.10 \mathrm{mmol})$ and benzaldehyde $(53 \mu \mathrm{L}, 1.0 \mathrm{mmol})$ in DCE $(1.0 \mathrm{~mL}, 0.5 \mathrm{M}$ in substrate). (E)-Crotylsilane (4:96 Z:E) 4 (118 $\mu \mathrm{L}, 0.750$ $\mathrm{mmol})$ was then added. The reaction was sealed and allowed to react for $24 \mathrm{~h}$. After workup, the product was purified by silica gel chromatography ( $96: 4$ hexanes:diethyl ether) to yield a yellow oil (63 mg, 78\% yield, 82\% ee). ${ }^{\mathrm{T}} \mathrm{H} \mathrm{NMR}\left(\mathrm{CDCl}_{3}, 400 \mathrm{MHz}\right): \delta$ 7.36-7.28 (5H, m), 5.82 $(1 \mathrm{H}, \mathrm{ddd}, J=17.6,10.0,8.4 \mathrm{~Hz}), 5.21(1 \mathrm{H}, \mathrm{d}, J=17.2 \mathrm{~Hz}), 5.20(1 \mathrm{H}, \mathrm{d}, J=10.4 \mathrm{~Hz}), 4.36(1 \mathrm{H}, \mathrm{dd}$, $J=8.0,2.8 \mathrm{~Hz}), 2.49(1 \mathrm{H}$, hex, $7.2 \mathrm{~Hz}), 2.15(1 \mathrm{H}, \mathrm{d}, 2.8 \mathrm{~Hz}), 0.87(3 \mathrm{H}, J=7.2 \mathrm{~Hz}) .{ }^{13} \mathrm{C}$ NMR $\left(\mathrm{CDCl}_{3}, 100 \mathrm{MHz}\right): \delta 142.5,140.7,128.3,127.7,116.9,78.1,16.9$. Optical Rotation: $[\alpha]_{\mathrm{D}}^{25}+97^{\circ}(\mathrm{c}$ $\left.1.0, \mathrm{CHCl}_{3}\right)$.

Optical purity is established by chiral HPLC analysis (Chiralcel OD (4.6 $\mathrm{mm} \times 250 \mathrm{~mm})$, 95:5 hexanes:isopropanol, $0.5 \mathrm{~mL} / \mathrm{min}, \lambda=220 \mathrm{~nm}$.); chromatograms are illustrated below for an $82 \%$ ee sample:

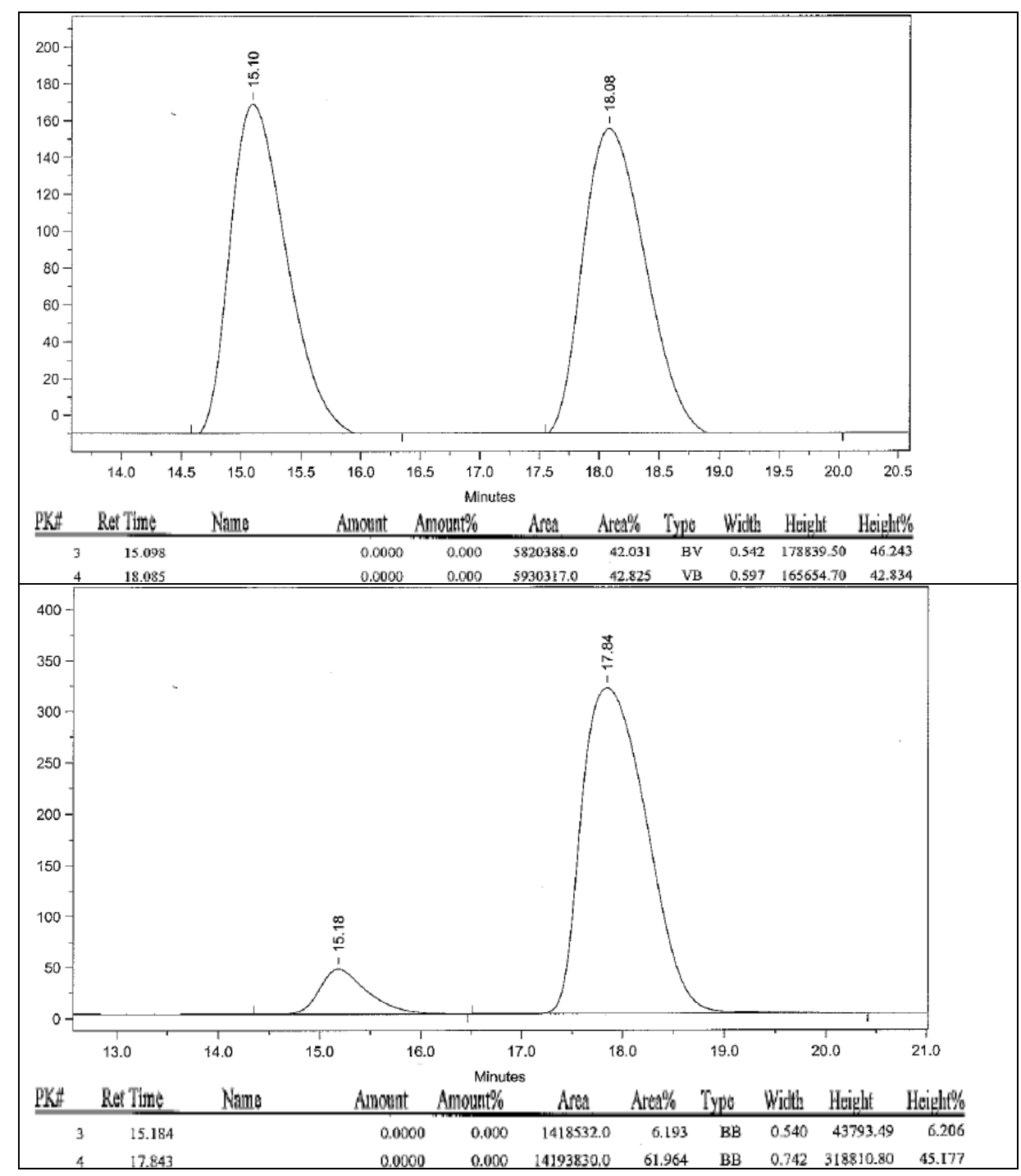

${ }^{18}$ Rotation, ${ }^{1} \mathrm{H}$, and ${ }^{13} \mathrm{C}$ NMR data are consistent with known product. See Ref. $6{ }^{\mathrm{b}}$. 
$\overbrace{\overline{\bar{M}}}^{\stackrel{\mathrm{OH}}{\mathrm{OH}}}$

(1R,2S)-2-Methyl-1-phenyl-3-buten-1-ol (7). ${ }^{19}$ The general procedure was followed with ligand $3 \mathbf{r}(63 \mathrm{mg}, 0.20 \mathrm{mmol})$, and benzaldehyde $(102 \mu \mathrm{L}, 1.0 \mathrm{mmol})$ in DCE (2.0 mL, 0.5 M in substrate). (Z)-Crotylsilane (97:3 Z:E) $5(233 \mu \mathrm{L}, 1.5 \mathrm{mmol})$ was then added. The reaction was sealed and allowed to react for $24 \mathrm{~h}$. After workup, the product was purified by silica gel chromatography (95:5 hexanes:diethyl ether) to yield a yellow oil (117 mg, 72\% yield, 76\% ee). ${ }^{1} \mathrm{H}$ NMR $\left(\mathrm{CDCl}_{3}, 400 \mathrm{MHz}\right): \delta 7.36-7.25(5 \mathrm{H}, \mathrm{m}), 5.77(1 \mathrm{H}, \mathrm{ddd}, J=$ 17.2, 9.6, $6.8 \mathrm{~Hz}), 5.08(1 \mathrm{H}, \mathrm{m}), 5.04(1 \mathrm{H}, \mathrm{m}), 4.62(1 \mathrm{H}, \mathrm{d}, J=4.4 \mathrm{~Hz}), 2.63-2.55(1 \mathrm{H}, \mathrm{m}), 1.97$ $(1 \mathrm{H}, \mathrm{br}), 1.02(3 \mathrm{H}, \mathrm{d}, J=6.4 \mathrm{~Hz}) .{ }^{13} \mathrm{C} \mathrm{NMR}\left(\mathrm{CDCl}_{3}, 100 \mathrm{MHz}\right): \delta 142.6,140.0,128.2,127.4$, 126.6, 115.6, 77.5, 44.9, 14.4. Optical Rotation: $[\alpha]_{\mathrm{D}}^{25}+21^{\circ}\left(\mathrm{c} 1.0, \mathrm{CHCl}_{3}\right)$.

Optical purity is established by chiral GLC analysis (Supelco Gamma Dex 120 (30 m x $0.15 \mathrm{~mm} \times 0.25 \mu \mathrm{m}$ film thickness), $90{ }^{\circ} \mathrm{C} 120 \mathrm{~min}, 0.5^{\circ} \mathrm{C} / \mathrm{min}$ to $130{ }^{\circ} \mathrm{C}, 15 \mathrm{psi}$ ); chromatograms are illustrated below for a $79 \%$ ee sample:

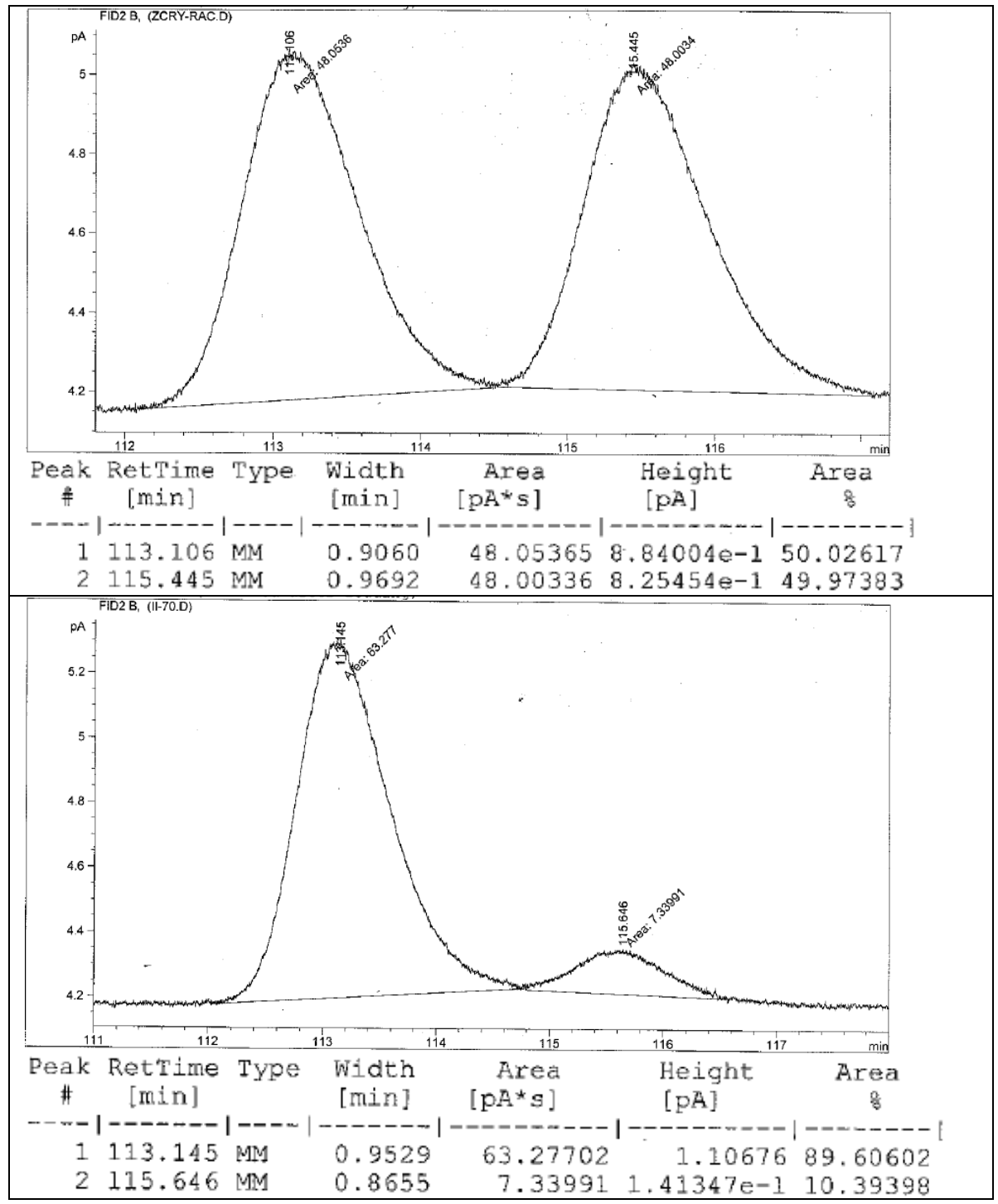

${ }^{19}$ Rotation, ${ }^{1} \mathrm{H}$, and ${ }^{13} \mathrm{C}$ NMR data are consistent with known product. See Ref. $6{ }^{\mathrm{b}}$. 